$$
\text { Roment hy OSTI }
$$

IS $-\mathrm{T}--1502$

DE91 007519

Indirect Fluorometric Detection Techniques on Thin Layer Chromatography and Effect of Ultrasound on Gel Electrophoresis

by

Ma, Yinfa

PHD Thesis submitted to Iowa State University

Ames Laboratory, U.S. DOE

Iowa State University

Ames, Iowa 50011

Date Transmitted: December 10, 1990

PRIPARED FOR THE U.S. DEPARTMENT OF ENERGY

UNDER CONTRACT NO. W-7405-Eng-82. 


\section{DISCLAIMER}

This report was prepared as an account of work sponsored by an agency of the United States Government. Neither the United States Government nor any agency thereof, nor any of their employees, makes any warranty, express or implied, or assumes any legal liability or responsibility for the accuracy, completeness or usefulness of any information, apparatus, product, or process disclosed, or represents that its use would not infringe privately owned rights Reference herein to any specific commercial product, process, or service by trade name, trademark, manufacturer, or otherwise, does not necessarily constitute or imply its endorsement, recommendation, or favoring by the United States Government or any agency thereof. The views and opinions of authors expressed herein do not necessarily state or reflect those of the United States Government or any agency thereof.

Printed in the United States of America

Available from

National Technical Information Service

U.S. Department of Commerce

5265 Port Royal Road

Springfield, VA 22161 
Indirect fluorometric detection techniques on thin layer chromatography and effect of ultrasound on

gel electrophoresis

\author{
Yinfa Ma \\ Under the supervision of Dr. Edward S. Yeung \\ From the Department of Chemistry \\ Iowa State University
}

Thin-layer chromatography (TLC) is a broadly

applicable separation technique. It offers many advantages over high performance liquid chromatography (HPLC), such as easily adapted for two-dimensional separation, for "wholecolumn" detection and for handling multiple samples, etc. However, due to its draggy development of detection techniques comparing with HPLC, TLC has not received the attention it deserves. Therefore, exploring new detection techniques is very important to the development of TLC. It is the principal purpose of this dissertation to present a new detection method for TLC--indirect fluorometric detection method. This detection technique is universal, sensitive, nondestructive, and simple. This will be described in detail from sections I through section $V$.

section I and III describe the indirect fluorometric detection of anions and nonelectrolytes in TLC. They are 
based on the displacement of fluorophore by the sample molecule, which cause a decrease of fluorescence. The negative signals can be monitored, i.e., analytes can be detected indirectly.

In section II, a detection method for cations based on fluorescence quenching of ethidium bromide is presented. Cations such as $\mathrm{Cu}^{2+}, \mathrm{Cr}^{3+}$ and $\mathrm{Hg}^{+}$can be separated on silica gel TLC plate and detected by quenching the red fluorescence of ethidium bromide.

In section IV, a simple and interesting TLC experiment is designed, three different fluorescence detection principles are used for the determination of caffeine, saccharin and sodium benzoate in beverages.

A laser-based indirect fluorometric detection technique in TLC is developed in section $V$. The new technique can be easily isad for quantitative measurements because of its two-dimension scanning capability. With this technique a detection limit of $6 \mathrm{pg}$ can be achieved. Section VI is totally different from sections I through $v$. An ultrasonic effect on the separation of DNA fragments in agarose gel electrophoresis is investigated. It is found that the ultrasound improves the separation of DNA fragments without degrading them, a phenomenon that is totally different from that of ultrasonic scission. 


\title{
Indirect fluorometric detection techniques on thin layer chromatography and effect of ultrasound on gel electrophoresis
}

\author{
by \\ Yinfa $\mathrm{Ma}$ \\ A Dissertation submitted to the \\ Graduate Faculty in Partial Fulfillment of the \\ Requirements for the Degree of \\ DOCTOR OF PHILOSOPHY \\ Department: Chemistry \\ Major: Analytical Chemistry
}

Approved :

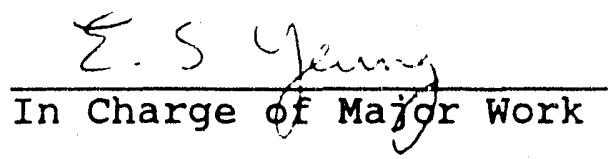
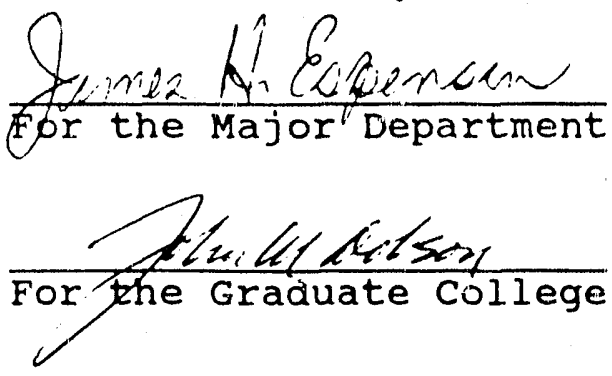

Iowa State University

Ames, Iowa

1990 
TABLE OF CONTENTS

Page

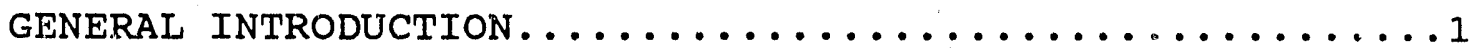

Introduction to Thin-Layer chromatography............

Overview of the TLC Detection Technique.............

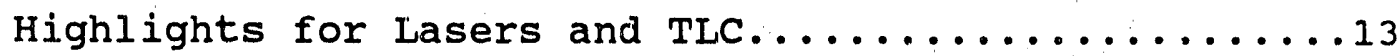

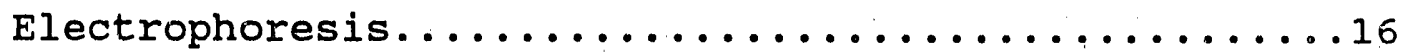

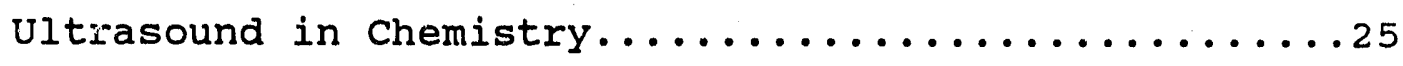

SECTION I INDIRECT FLUOROMETRIC DETECTION OF ANIONS

IN THIN-LAYER CHROMATOGRAPHY..........28

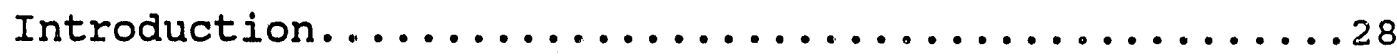

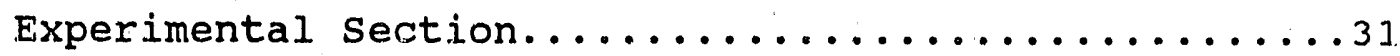

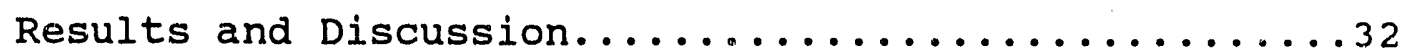

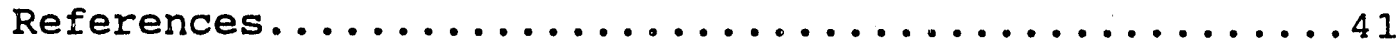

SECTION II DETECTION OF CATIONS SEPARATED BY THIN-

LAYYER CHROMATOGRAPHY BY FLUORESCENCE

QUENCHING OF ETHIDIUM BROMIDE.........43

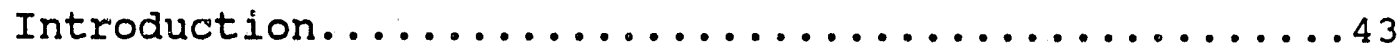

Experimental............................ 45

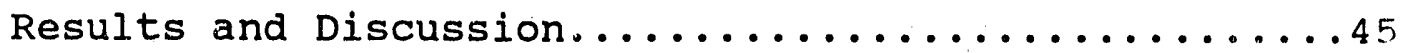

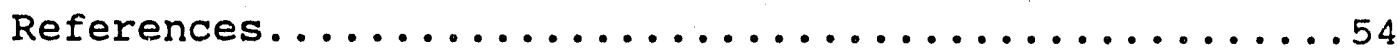

SECTION III INDIRECT FLUORIMETRIC DETECTION OF

NONELECTROLYTES IN THIN-LAYER

CHROMATOGRAPHY..................... 55

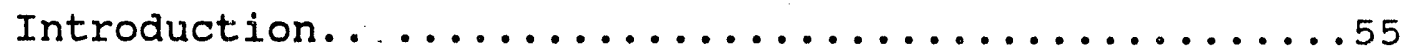

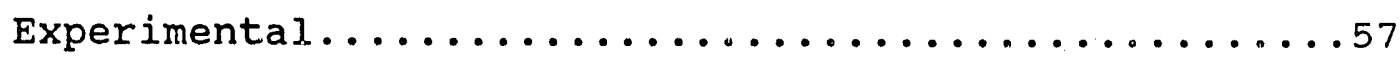

Results and Discussion...................60 
Conclusion.............................

References.............................. 74

SECTION IV DETERMINATION OF COMPONENTS IN BEVERAGES

BY THIN-LAYER CHROMATOGRAPHY --

AN UNDERGRADUATE ANALYTICAL CHEMISTRY

EXPERIMENT . .....................77

Introduction.......................... 77

Experimental........................... 78

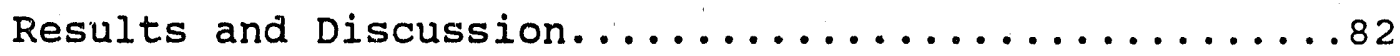

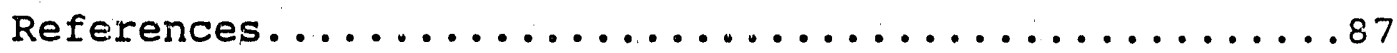

SECTION V LASER-BASED INDIRECT FLUOROMETRIC DETECTION AND QUANTIFICATION IN THIN-

LAYER CHROMATOGRAPHY................... 89

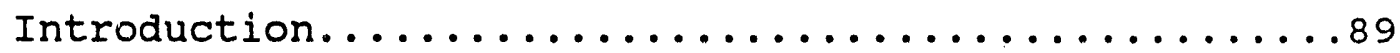

Experimental Section......................

Results and Discussion...................96

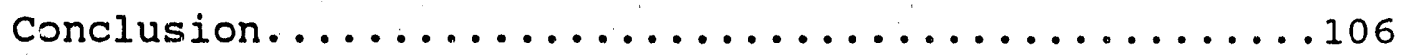

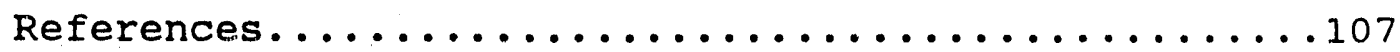

SECTION VI THE EFFECT OF ULTRASOUND ON THE

SEPARATION OF DNA FRAGMENTS IN

AGAROSE GEL ELECTROPHORESIS...........110

Introduction...........................110

Experimental section.....................111

Results and Discussion...................117

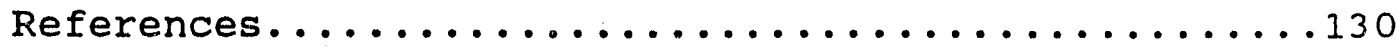

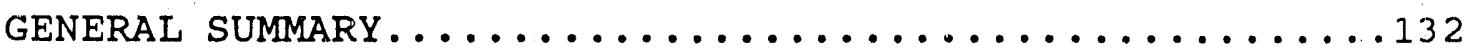

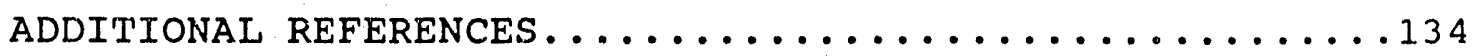

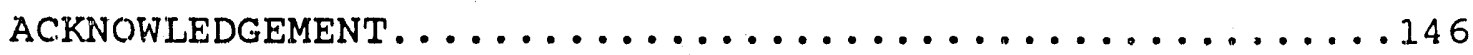


GENERAL INTRODUCTION

Introduction to Thin-Layer Chromatography

Nowadays, despite developments in analytical chemistry that link scientists to extremely sophisticated devices, chromatography plays a very important role among analytical techniques. It is rare to see a laboratory without chromatographic equipment. Quality control, production control, and basic research are some of the filds in which chromatography is used.

Thin-layer chromatography (TLC) is a liquid chromatographic technique, and is the oldest form of chromatography. TLC was first referred to in 1938 when two Russian workers, Izmailov and Schraiber (1), reported what they called drop chromatography on horizontal thin layers. There was almost no progress until 10 years later, when two American chemists described the separation of terpenes in essential oils by TLC (2). TLC, as it is presently known, began to attract attention through the work of Kircher and his associates, starting in 1951 (3-5). However, the lack of apparatus for coating the plates and available media made TLC not generally accepted in its early years. Actually, it was not until 1958, when stahl (6) described equipment and efficient sorbents for the preparation of 
plates, that the technique of TLC became practical. Stahl's book (7) and Kirchner's book (8) really led I'TiC to a new stage. With the development of high performance TLC (HPTLC) plates, the introduction of new stationary phases and the instrumentalization of virtually all aspects of the technique, TLC has now been transformed into a modern, sensitive, and one of the most frequently described separation techniques in qualitative as well as quantitative analysis. Several books $(9-11)$ on TLC will give a greater impetus to development in the field.

A TLC separation system consists of two components: a stationary phase and a mobile phase. The stationary phase is composed of uniform thin layers of sorbent or selected media which are used as a carrier medium. The sorbent is applied to a backing as a coating to obtain a stable layer of suitable size. Various stationary phases and their characteristics have been clearly described (11, 12). The mobile phase is a eluent which is used for carrying the sample to move through the stationary phase. The nature and chemical composition of the mobile phase is determined by the type of substance to be separated and the type of sorbent to be used for the separation. The composition of a mobile phase can be as simple as a single, pure solvent or as complex as a three- or four-component mixture containing definite proportions of chemically 
different substances. A sample mixture (solutes) is introduced to the bottom surface of the sorbent layer as a spot. A solvent (mono or multicomponent) is passed through the TLC plate from bottom to the top by capillary forces, where the separation occurs. Due to the different interaction between the sample components and the stationary phase, the molecules of the sample will move through the stationary at different speed, which lead to a difference in the migration of eash component.

Even though high-performance liquid chromatography (HPLC) has shown a remarkable growth in recent years and has developed into a modern and powerful technique in chemical analysis, the use of TLC has not declined noticeably and currently shows signs of a resurgence in popularity. This is not only due to the introduction of new stationary phases and greatly improved instrumentation for TLC, but also due to a number of advantages of TLC over HPLC, which has been described by several authors (12-16). The advantages of HPTLC over HPLC are summarized in Table I. This does not claim that TLC should replace HPLC as a separation tool, TLC has its own shortages, such as lower resolving power, higher detection limits in many cases, unsuitability for solvent gradients, and so on. In essence, the two techniques complement one another and therefore selection of the methods should be based on 
Table I. Advantages of HPTLC over HPLC

\begin{tabular}{|c|c|c|}
\hline Parameter & HPTLC & HPLC \\
\hline Simultaneous sample analysis & yes & no \\
\hline $\begin{array}{l}\text { Sample simultaneously available } \\
\text { for detection }\end{array}$ & yes & no \\
\hline Multiple scan of same separation & yes & no \\
\hline Sample capacity & high & low \\
\hline Column poisoning & no & yes \\
\hline Two dimensional separation & yes & difficult \\
\hline $\begin{array}{l}\text { Unrestricted choice of solvents } \\
\text { by consideration of detcotion }\end{array}$ & yes & no \\
\hline System equilibration time & short & long \\
\hline Cost & lower & higher \\
\hline $\begin{array}{l}\text { Easy change of solvents to } \\
\text { optimize selectivity }\end{array}$ & yes & no \\
\hline Disposable & yes & no \\
\hline
\end{tabular}

type of problem to be solved. For example, for a complicated sample mixture, HPLC would be a choice because of its high separating power; when a high throughput of 
similar sample is required, the HPTLC can provide considerable savings in time.

TLC itself can be divided into two categories: conventional TLC and HPTLC. The main difference between both is that HPTLC has a smaller and uniform sorbent particle size, which give higher numbers of usable theoretical plates (5000), higher separation power, shorter separation time and lower detection limits. A comparison of the both has been described in detail $(13,16)$. Thus, HPTLC represents a considerable advance in the practice of TLC.

Overview of the TLC Detection Technique

The detection techniques for TLC and liquid chromatography (LC) are quite different. LC is performed in a sequential manner so analytes are detected as they elute. In TLC, detection is a completely independent step and is carried out in the opaque nor-uniform solid particles with large scattering interferences. Therefore, some detection techniques that work very well for HPLC, such as a refractive index detector (17), a polarimetry detector $(18 \mathrm{a}-18 \mathrm{C})$ and all different kinds of electrochemical detectors (19-20b), do nct work for TLC. TLC detection conventionally is limited to only a few 
optical methods such as absorption and fluorescence, except the use of radioactive technique. The detection limit is also hampered by the highly scattering background of the thin layer material. This probably is the main reason that TLC lagged behind in the early years. In recent years, various advanced instruments $(21-22)$ and detection techniques really bolstered the TLC method. Detection techniques fur TLC will be briefly discussed in this overview.

\section{Visual detection}

The success of separation of colored compounds is usually monitored visually. Such compounds absorb a particular portion of the polychromatic (white) light in the visible wavelength range. The remaining radiation (complementary radiation) is reflected and detected by the eye; it determines the color of the substance zone. For colorless compounds, usually spraying reagents are used to make them visible on the chromatogram. spraying reagents can be divided into two classes: (1) general reagents, which detect a large number of different types of compounds, and (2) specific reagents, which indicate the type of compound or functional group present. Up-to-date, hundreds of spraying reagents have been found for the detection of different compounds $(23-25)$. 
Visual detection can only be used for qualitative and semiquantitative detection, and the search for a proper spraying reagent could be a tedious job.

\section{Fivorescence detection}

Fluorescence spectroscopy is a widely applied detection technique in both TLC and HPLC. It is especially useful for the quantitative determination of molecules at trace levels, and it is one of the easiest techniques for achieving low limit of detection (LOD), because of its high sensitivity and larger linear dynamic range (26-27). The basic principle of fluorescence detection is that molecules that absorb radiation are raised to an excited state, when returning to the ground state, they emit the absorbed energy instantaneously as radiation energy in the form of fluorescence (28). The radiation emitted is usually longer in wavelength (i.e., lower in energy) than the incicient light (Stoke's law). Unfortunately, fluorescence detect on suits only fluorescent compounds or those compounds which can be derivatized to become fluorescent. Therefore, it is not a universal detection method in spite of its high sensitivity and LOW LOD. 


\section{Al sorbance detection}

In situ densitometry is the most commonly used method to do quantitative determination on $\operatorname{TLC}(11,13,29)$. The TLC plate is scanned by a beam of light with predetermined wavelength in the mode of reflectance, transmission, or both simultaneously. Light striking a spot on the plate will undergo absorption so that the light transmitted or reflected is diminished in intensity at those wavelengths, forming the absorption profile of the spot. The measurement of the signal diminution due to this absorption by the spot provides the mechanism for in situ quantization. Since TLC plates are opaque and scatter light strongly, absorption measurements cannot be expected to obey the Beer-Lambert law. The Kubelka-Munk equation has to be used for correction. Its derivation can be found elsewhere $(11,30)$. This method causes higher errors (about $3 \%$ ). The reason for this is that absolute values of transmittance and/or reflectance are necessary for application of Kubelka-Munk equation. However, it is difficult to find a good reliable reference for absorption measurements in scattering media. The change in layer thickness and the optical properties of the adsorbent can cause serious background interferences and result in a less favorable signal-to-noise ratio. The LOD for this method is about $10 \mathrm{ng}$ to $1 \mu \mathrm{g}$, varying with different samples. 
Flame ionization detection (FID)

Since the late 1960s, attention has been focused on FID as a quantitative detection technique of TLC (31-32). TLC is carried out on narrow quartz plates coated with silica gel. After removal of the develsping solvent, the TLC plate called "chromatoplate" is encased in a rectangular quartz chamber and driven through a pyrolysis furnace, while a carrier gas, nitrogen or helium, flow through the chamber to a FID. Thereby, the substances separated on the chromotoplate are consecutively vaporized by pyrolysis and/or evaporation and the gaseous products are swept by the carrier gas to the FID, which records each fraction as a separate peak. But the pyrolysis furnace which was used to vaporize the samples could not provide the satisfied lateral resolution on the chromatoplate. Thereafter, a tubular TLC called "chromatotube" came into use, which considerably improved the above technique (3334). The most popular TLC-FID system is coated-Rod TLC-FID system (35), which is called "chromarod". TLC is carried out on an adsorbent-coated quartz rod; the rod is then moved through the flame jet of an FID; ion signals are collected by the collector electrode which is located on the top of flame. The powerful FID method makes this technique grow very quickly, and it has been developed into a independent TLC method (36-38). 


\section{Indirect detection}

In recent years, indirect detection method has become a powerful detection tool for IC (39-48) and capillary zone electrophoresis (CZE) (49-53). The basic principle of indirect detection is that the detector responds to some physical property of the eluent, so that a constant background signal is maintained at the detector when no analytes exist. When analyte elutes, it displaces some amount of the eluent and is recorded as a "vacancy" peak. Indirect detection began with indirect photometric chromatography (IPC), which was initiated by Small and Miller (54). An UV-absorbing counter-ion was added in the mobile phase to compete with UV-transparent, injected analyte for the ion exchange sites. As the UV-transparent analyte elutes off the column, it replaces the UV-absorbing counter-ion in the effluent which leads to a decrease in absorbance at the detector and produces a negative peak. Later on, indirect polarimetry (55-56) and indirect fluorescence $(40,50,57)$ detection methods for $L C$ and CZE were well developed. Its theoretical background, requirements, and sensitivity have been clearly described $(45,58-59)$.

Based on the same principle, an indirect fluorometric detection method on TLC is proposed in this dissertation. 
Briefly, a TLC plate is treated with a fluorophore, when the samples are developed by a fluorescent solvent, dark spots being produced by displacement of the fluorescent compounds by analytes are detected. The details about this method will be described in sections $I-V$.

The indirect fluorometric detection on TLC has quite a few advantages over other detection methods. (1) It is a universal detection technique and has little requirement for the exact nature of the samples. Therefore, the compounds that give low response on UV absorption, fluorescence and other detectors can be detected by this technique. The development of this technique will expand the application scale of TLC. (2) It is fast and simple. Especially for colorless compounds which do not absorb in the UV, the tedious sample manipulation (e.g., chemical derivatization) and search of spraying reagents are avoided. (3) It is sensitive. In indirect fluorescent detection, we are the master to choose a fluorophore, so trat a sensitive fluorophore with a high displacement ratio (58) will achieve very low LOD. A 6 pg IOD has been demonstrated, which has a same LOD level as that of direct fluorometric detection. (4) It is an non-destructive detection technique. Since no chemical manipulation is involved in this method, the analytes can be collected for further use. 
other miscellaneous detection methods

In addition to above introduced TLC detection methods, some others also appeared in recent years. Photoacoustic spectrometric (PAS) technique $(60-62)$ is one of them. It is based on absorption of light. When a molecule absorbs light, it may subsequently release the energy as heat (a nonradiative process). In PAS experiment, the sample is held in an enclosed container, this released heat will actually create sound waves that can be detected by a microphone or piezoelectric crystal. The amount of heat produced (and the intensity of the sound wave) is proportional to the amount of sample. Photothermal deflection (PTD) $(63-64)$ is a variation of the PAS. In this technique, one high-power laser is focused on the TLC plate surface, where a sample spot will absorb the light. The heat warms up the air over the spot and creates a "thermal lens", an area of gas that is warmer, and has a different refractive index. If a low-power laser is directed through this region, it will be deflected when thermal lens forms. The intensity of signal which changes with the position of laser beam deflncted is proportional to the concentration of sample. Mass spectrometry (MS) has been used for TLC detection recently (65-69). Simply, the sample on the TLC plate is ionized by fast atom bombardment (FAB) or a cesium ion gun and is drawn into the 
spectrometer by ion focussing system. The major advantages of MS as a TLC detector are that it can provide structural and molecular weight information, it is sensitive and has a high spatial resolution. Computer-Based Videodensitometer is also introduced to be a TLC detector $(10,70)$. This detection method is very useful for on-line detection and is easy to correct background fluctuation. Again, a detection method in this category responds to a specific property of the analyte. If the analyte does not possess that specific property, difficulties in detection may arise. In this case, indirect detection method introduced above will be a good choice.

\section{Highlights for Lasers and TLC}

Since the laser was invented in 1960s (71), its analytical applications have grown very rapidly $(72-76)$. The reason for this fact is due to the advantageous properties of laser light. First, the wavelength tunability enables radiation at any wavelength in IR, visible, and UV spectral regions to be obtained. This makes it possible to study almost any quantum transition of atoms and molecules. Second, the high intensity of laser radiation can transfer much more fraction of atoms or mrlecules to an excited state. This is very useful to 
fluorescence detection, because fluorescence intensity is proportional to the strength of the excitation light. Most laser, will easily produce at least 100 times as many photons as a conventional light source, this means that fluorescence intensity may increase 100 times. Third, tre monochromatic output $(<1 \mathrm{~nm})$ allows measurement of spectra with almost any required resolution and selective excitation of particular atoms or molecules in mixtures. Fourth, the collimated output makes focussing easier or not even necessary, and can be used for a spectral probing or focused the radiation onto a small area for local analysis. Also, a collimated laser beam is very easy to be used for scanning. Finally, the pulsed output which produces a large number of photons over a very short time period (nsec. or psec.) allows us doing time-resolved spectroscopic studies.

Laser-based detection methods for TLC was introduced by Berman and Zare in 1975 (77). Subnanograms of aflatoxins can be detect by laser fluorescence. Since then, various laser-based detection techniques sprouted up. Laser-induced fluorescence detection method became more mature due to the improvements in instrumentation and background correction. Picogram level LODs can be easily achieved (78-79). Laser-based PAS (60-61) for TLC detection is also a new technique. Most PAS-TLC 
experiments utilize a xenon lamp, rather than a laser. In that case, the detection limits usually stay at $\mu \mathrm{g}$ level. The laser-based PAS, however, can reach subnanograms LOD very easily $(50)$. Since lasers are superjor in producing the PAS effect, the future of the PAS-TLC technique will count on lasers as a light source. Laser-based PTD is used for TLC detection in $1984(63-64)$. LOD of low pg range is reported. Laser-based nonlinear luminescence detection is a less common spectroscopic phenomena. Two-photon excited fluorescence (TPEF) and sequentially excited fluorescence (SEF) have been reported in LC detection (80-81). The TPEF process involves the simultaneous absorption of two photons to reach an excited level. This excited molecule then emits a photon (fluorescence) with an energy greater than (shorter wavelength than) the exciting radiation. This is the opposite of the noxmal fluorescence methods. The SEF process involves sequential absorption of photons to a very high excited state, followed by fluorescence. These two processes can be very useful in TLC detection, since they offer additional selectivity in fluorescence detection quite unlike any other. These techniques have now been elegantly applied to TLC analysis (82). The pg range of LOD for oxadiazole compounds has been reported. A laserbased TLC detection method based on laser pyrolysis and laser desorption is also developed (83-84). A high power 
laser is used to vaporize the samples separated on TLC plate into gas phase, then the sensitive and universal GC detectors are facilitated for TLC detection. The LOD of $\mathrm{ng}$ level is reported. Laser-based indirect fluorometric detection on TLC presented in this dissertation will push applications of TLC to a new stage. It is not only universal, but also sensitive. A $6 \mathrm{pg}$ level LOD is reached, which is 100-fold lower than visual detection, and is in the same LOD range as that of direct laser-based fluorescence detection. For some sample, it may be more sensitive than other laser-based techniques.

Based on the above introduction on laser-based detection techniques on TLC, one can imagine that laser technology and optical spectroscopy will play a major role in TLC detection, and make the TLC application be more wider and more popular.

Electrophoresis

Electrophoresis is a separation method of charged molecules based on differential migration in an applied potential field. It represents one of the most widely used analytical separation techniques in chemistry. The power of the method, which spans from the separation of small ions to biopolymers and even whole cells, is utilized 
especially in biochemical and biomedical areas. Well. established methods based on isoelectric focusing and gel sieving electrophoresis are quite effective in their own right and therefore represent the work horse in protein and polynucleotide research.

The technique was first described in 1930 in the doctoral thesis of Arne Tiselius and publisned in the scientific literature in 1937 (85). Since Tiselius's report of "moving boundary electrophoresis" of protein solutions many improvements and elaborations of the basic technique have been introduced. A great number of reviews, books and monographs discussing the present state of the art of gel electrophoretic methods have been published (86$89)$.

The formats of electrophoresis can be divided into two major categories: free sollition and gel electrophoresis.

\section{Gel electrophoresis}

In gel electrophoresis, anticonvection stabilizers are used. The original intent of the use of stabilizers in electrophoresis was to suppress the thermally driven convection currents in the electrophoretic medium. While this remains an important function of stabilizers, several additional benefits have been realized. Microporous gels permit separations based on molecular size through a 
sieving action (89). Gels create an "anchoring" point for buffering ions allowing immobilized (stable) pH gradients. And perhaps most important of all, stabilizers offer a convenient format for electrophoresis, where "strips" and "slabs" can be easily stained, destained, and otherwise manipulated in ways unthinkable for free solutions. Due to these significant advantages, a large number of electrophoresis is usually done with the use of a stabilizer. This can be proved by looking through the pages of journals devoted to biochemistry and molecular biology, there one can find great numbers of photographs of gel electrophoretic separations.

Even though the actual mechanism of the molecular weight separation of macromolecules in a gel is not clear (90-91), people seem to accept the mechanism proposed by ogston based on a random meshwork of fibers (92). The electrophoretic mobility of a macromolecule is proportional to the volume fraction of the pores of a gel that the macromolecule can enter $(90,93-94)$. Since the average pore size decreases with increasing gel concentration $(90$, 93-95), electrophoretic mobility is predicted to decrease with increasing gel concentration and with increasing molecular weight.

For gel electrophoresis, two types of gel matrices are commonly used: Polyacrylamide and agarose. By controlling 
the concentration of acrylamide and the relative proportion of the cross-linking agent bis-acrylamide, gels can be formed with well-defined molecular sieving properties (89). Polynucleotides may be run on these gels with spectacular results (96). Actually, biochemists always use this powerful and efficient method for DNA sequencing. The separation of proteins on polyacxylamide gel almost always involves the use of denaturing ionic detergents such as sodium dodecyl sulfate (SDS). Proteins will "bind" a limiting amount of 1.4 grams of SDS per gram of protein. In this case, all proteins have a same charge to mass ratio, the separation, therefore, is just based on shape and size. The proteins having lower molecular weight are easier going through the gel and migrate faster, the larger ones run slower. With two-dimensional electrophoretic approach, Neidhardt and Phillips (97) have demonstrated that about 30,000 proteins can be separated. This is a phenomenal number when compared with even the highest resolution of chromatographic methods. Two dimensional polyacrylamide gel electrophoresis is so powerful that people use it for genetic analysis and monitoring of celi proliferation (98). For molecules of molecular weight in excess of one million, however, even the lowest concentration of polyacrylamide which forms a physically stable gel (about 3\%) yields pores too small to permit 
migration. In this situation, agarose gel will be a choice. Agarose gels can form larger pores than acrylamide, and permit separation of much larger molecules. The separation of whole chromosomes from yeast has been reported (99). The molecular weights of these chromosomes span the range of $3 \times 10^{7}$ to $1 \times 10^{9}$. Separation of such large DNAs by chromatographic methods is essentially impossible. In general, polyacrylmide gel is used more in protein separations, agarose gel is mainly used in DNA research. But this division is not strict, a combination of both gels has been reported (89).

Detection techniques in gel electrophoresis include: staining, autoradiography and on-line detectior. A variety of dyes can be used for protein staining (89). One of the most sensitive and frequently used dyes is coomassie Blue. For DNA, Ethidium Bromide is always used. In autoradiography detection mode, a radioisotope (e.g., ${ }^{32} \mathrm{p}$, ${ }^{131} \mathrm{I},{ }^{14} \mathrm{C}$ or ${ }^{3} \mathrm{H}$ ) has to be incorporated in the protein or DNA molecule. On-line detection for gel electrophoresis has not been reported yet, but recent advances in computerAssisted Charge-Coupled Device ( $C C D$ ) cameras make it possible to monitor the separation process (100). Also, indirect fluorometric detection can be used (101), this technique is much simpler than staining and autoradiographic methods. 
Gel electrophoresis can be influenced by gel. concentration, temperature and electric field (102). In order to take the advantage of high electric field and minimize the affect of temperature gradient, high performance capillary gel electrophoresis system (HFCGE) has been emerged recently (103-105). The advantages of HPCGE are high resolution, time saving, sensitive, lower LOD and easy to be automated. Also, more on-line detection mode, such as fluorescence, absorption and indirect fluorescence methods, cin be conveniently used.

In addition, gel electrophoresis can also be influenced by ultrasound. Ultrasound will increase the separation of DNA frägments in agarose gel electrophoresis. The details will be discusser in section VI.

\section{Free solution electrophoresis}

Here only high performance capillary zone electrophoresis (HPCZE) is discussed, because the free solution electrophoresis in larger tubes seems to be out of date due to its poor resolution caused oy temperature gradient in the separation medium. When the electrophoresis is running in a tube, Joule heat is generated uniformly throughout the tube cross section but is dissipated through the tube walls. This results in a temperature gradient across the tube with the fluid in the 
center being warmer than the fluid at the wall. This temperature gradient results in a density gradient (leading to a convective flow) as well as viscosity and $\mathrm{pH}$ gradients. These gradients will lead to spreading of electrophoretic zones. However, because the capillary has a very small diameter ( 5 to $50 \mu \mathrm{m}$ ), and the temperature gradient between the fluid in the center and at the wall is proportional to the square of the tube's inside diameter (106-107), HPCZE makes this effect almost ignored, and permits the use of high potentials for separation. The use of high potential fields leads to extremely efficient separations with a dramatic decrease in analysis time. In addition, HPCZE has other advantages just like HPCGE. Detectors for column liquid chromatography, which can provide many very sensitive detection modes, can also be used for HPCZE. The ultrasmall volume flow rates obtainable in HPCZE permit sampling from picoliter environments. It is well-suited for automation because it allows on-line detection and automation of sample loading. The basic principles of HPCZE are quite simple. A small plug of sample solution is introduced into sne end of a fused silica capillary tube containing an appropriate electrolyte. Component molecules in the injected sample then migrate at different rates along the length of the capillary separation channel under the action of a large 
electrical field, and the molecules are detected as they approach the other end of the capillary by one of the several on-line detection system (108). The detailed description on separation mechanism, instrumentation can be found in many places (109-110).

The commonly used detectors in HPCZE are UV absorption and laser induced fluorescence. However, sample derivatization is required for some molecules of interest. In this case, an indirect fluorometric detection technique can be used (49). Recent advances have allowed the use of several nonoptical detection methods, including mass spectrometry (112-113), electrochemistry (114), and conductivity (115). Although each detection system has its Iimitation, the variety of techniques available has expanded the scope of systems that can be examirad with HPCZE. Wallingford and Ewing (116) have clearly evaluated each kind of detector.

The applications of HPCZE have no limitations actually. Simple anions and cations, peptides, polynucleotides, sujars and even nonionic samples can be separated and detected. The nonionic compounds can be separated by micellar electrokinetic capillary chromatography (MECC) (117-118). In MECC, ionic surfactants, such as sodium dodecyl sulfate (SDS), are added to the operating buffer at concentrations exceeding 
the critical micelle concentration (CMC). At this level, surfactant monomers tend to form roughly spherical aggregates, or micelles, with the hydrophobic tail groups oriented toward the center and the charged head groups along the outer surface. Micelles are dynamic structures and are always in equilibrium with surfactant monomer. Actually, the micellar solutions of ionic surfactants work as a moving stationary phase. When a high voltage is applied to the system, both electroosmotic flow of the whole solution and electrophoretic migration of micelles are induced. The directions of electroosmotic flow and electrophoretic migration of micelles are generally opposite, and the magnitude of electroosmotic flow is greater than that of micelle migration. Therefiore, solutes can partition between free solution and the interior of the micelles, resulting in retention based on differential solubilization by the micelles. Hence, separation of nonionic compounds is possible. A comprehensive review of applications of HPCZE has been published (119) .

originally, HPCZE did separations just based on charge, and could not do size sorting. Recently, several reports have demonstrated that HPCZE can be used for DNAS separation based on size (120-121). The main point is that linear polymers such as hydroxypropylmethylcellulose (HMC) and polyethylene glycol (PEG) are incorporated into the 
electrophoresis buffer, by the selection of the appropriate linear polymers, separation of DNAs over a wide range of molecular weights can be achieved. The real working mechanism needs further investigation.

\section{Ultrasound in Chemistry}

Ultrasound is used to describe sound wave frequencies within the range 20 to $10,000 \mathrm{kHz}$. The basis for the present-day generation of ultrasound was established as far back as 1880 with the discovery of the piezoelectric effect and its inverse by Curie and Curie (122). Most modern ultrasonic devices rely on transducers which use the inverse effect, i.e., the production of a change in dimension of certain materials by the application of an electrical potential across opposite faces. If the potential is alternated at high frequencies the crystal converts the electrical to mechanical energy--ultrasound. The application of ultrasound was first used in estimatirig the depths of water (123). Since then, the use of ultrasound expanded very quickly, including irdustries (welding, cleaning) (124), biology (homogenization, cell disruption and scission of DNA) (125), and medicine (diagnosis, surgery, dental applications) (126) . 
Since the use of uitrasound in chemistry at $1940 \mathrm{~s}$ (127), various applications of ultrasound in chemical processes have been reported (128). These include synthesis (organic, organometaliic and inorganic), polymer chemistry (degradation, initiation, and copolymerization), and some aspects of catalysis. The area which uses ultrascund most is organic synthesis. Organic chemists use ultrasound to increase chemical reactivity. The main mechanism is that cavitation in liquid induced by ultrasonic waves creates a series of gas and vapor filled bubbles-microbubbles. On collapse, these vapors are subjected to the enormous increases in both temperature and pressure. Under such extremes the solvent and/or reagent suffers fragmentation to generate reactive species of the radicals. In addition, the shock wave produced by bubble collapse could act to disrupt solvent structure which could influence reactivity by altering solvation of the reactive species present. A very good review on chemical ultrasonics has summarized the applications of ultrasound in chemical reactions (129).

The applications of ultrasound in analytical chemistry are not as popular as those in organic chemistry. Since Dunken et al. (130-131.) demonstrated the use of ultrasonic nebulization in flame atomic spectrometry in 1964, various types of ultrasonic nebulizers have been designed for 
different purposes (132-134). In the sonochemistry symposium of 1986, Asher (135) demonstrated that ultrasound can be used for lic lid identification, measurement of liquid concentration, identifying and analyzing gasies by determining the velocity of ultrasound. The main point is that the ultrasound speed changes with direct melia. When ultrasound passes different liquids or gases, the velocities are different, which are lise standards for identifying a liquid or a gas. With the same principle, when ultrasound passes a series of solution, the velocity of ultrasound will linearly decrease with the increase of concentration, which can be used for concentration determination. However, none of them is accurate, and need to be calibrated by other methods. In 1987 , the effect of ultrasonic agitation in high performance size exclusion chromatography was investigated (136). It was found that peak widths of a variety of compounds increased while retention is unchanged under the ultrasonic agitation.

In this dissertation, the effect of ultrasound on gel electrophoresis is presented. When directing ultrasound energy to the tube gel while electrophoresis is running, it is found that the ultrasound improves the separation of DNA. fragments without degrading them. This phenomenon will be important to the bioanalysis in the future. 
SECTION I

\author{
INDIRECT FLUOROMETRIC DETECTION OF ANIONS \\ IN THIN-LAYER CHROMATOGRAPHY
}

Introduction

Thin-layer chromatography (TLC) is a broadly applicable separation technique. With the recent growth of interest and products for high-performance liquid chromatography (HPLC), TLC has not received the attention it deserves. A major factor is that the approach is still not highly "instrumental" compared to HPLC, both in the separation step and the detection step, even though much progress has been made (1). TLC provides many advantages as an analytical technique. It is easily adapted for twodimensional separation (2), for "whole-column" detection (3), and for handling multiple samples. As a complementary technique to HPLC, one can use TLC as an initial screening step to optimize conditions. This is because of the ease of equilibration with a new mobile phase, the speed of development, the freedom from contamination and carry over, the applicability to highly retained solutes, and the low cost of stationary and mobile phase used. 
Detection in TLC lags behind the corresponding technology in HPLC. The two-dimensional geometry of the spots limits the path length available for absorption photometry, although nanogram levels are readily detectable. The sensitivity problem can be partially solved by using photothermal spectroscopy (4) or photoacoustic spectroscopy (5). Nonlinearity still exists because of the scattering nature of the substrate (6), and the Kubelka-Munk function is needed for correction. If the analyte fluoresces, laser fluorometry has been shown to. allow detection of the order of $5 \times 10^{-14}$ mol per spot (7). All of these require derivatization for the majority of analytes because of the lack of absorption, either before or after development. Besides being an undesirable complication, unreliable chemistry and irreproducible operation can drastically affect quantitation.

Recently, several indirect detection methods for LC have been demonstrated $(8-10)$. Briefly, the detector responds to some physical property of the chromatographic eluent. So, there is a constant background signal generated at the detector when no analytes are present. When the analyte elutes, it displaces an equal amount of the eluent at the detector. Even though the detector does not respond to the analyte, the lower elueut concentration at the detector causes a decrease in signal. The analyte 
can then be monitored as a negative signal, i.e., indirectly. So, it should be possible to devise a detection method for TLC based on indirect fluorometry. It may then be possible to extend the advantages for fluorescence detection to species that do not themselves absorb or fluoresce.

There is a technique for TLC quantitation known as fluorescence quenching (11). In fact, commercial TLC plates are available with a fluorophore already incorporated for this purpose. It is really a misnomer because rarely does the analyte actually change the fluorescence quantum yield of the fluorophore. In reality that is only a modified absorption technique. The analyte absorbs the excitation light (or the emitted light), and depletes the amount of fluorescence. The advantage is the elimination of UV optics in the observation of emission, so that direct visualization is possible for UV chromophors. sensitivity is at best comparable to UV transmission or UV reflectance spectrometry. In this article, we describe the detection of nonfluorescing and nonabsorbing anions. Displacement of a fluorescing (background) eluting ion by the analyte ion due to electroneutrality gives rise to the response. 


\section{Experimental section}

The setup for TLC is conventional, with development in the vertical direction. Samples are applied by a microsyringe calibrated in $0.1-1$ l units. Cetyltrimethylammoniun bromide (cetrimide) was obtained from Aldrich (Milwaukee, WI). Samples and various solutions are prepared from reagent grade chemicals (Fisher, Fair Lawn, NJ), the former being the sodium salts of the respective anions. TLC plates used are either polyester-backed [(diethylamino)ethyl]-tert-amine cellulose (Anspec, Warrenville, IL, Whatman 100-1m CEI,300 DEAE) or glass-backed silica gel (Alltech, Deerfield, IL, Adsorbosil-plus 1 ), cut to 5 -cm widths. Pretreatment of TLC plates is accomplished by immersing the plates for predetermined time intervals in solutions in a beaker, rinsing with deionized water, and drying with a heat gun. Visualization is aided by a UV lamp (Cole Palmer, Chicago, IL, 9815, $312 \mathrm{~nm})$. Photographs are unretouched ard are taken by a 35-mm camera using Plus-X film (Kodak, Rochester, $\mathrm{N}:$ ) and a macrolens with a $1: 4$ or lower magnification, so that the plate fills most of the frame. The same UV lamp is used for excitation. 


\section{Results and Discussion}

DEAE cellulose plates are natural anion exchangers at neutral $\mathrm{pH}$. They can be used without modification in this scheme. Analogous to equilibration of the ion-exchange column in nonsuppressed ion chromatography (IC) (12), the commercially available plates are first immersed in a solution of $5 \times 10^{-5} \mathrm{M}$ sodium salicylate. This is to avoid depletion of the concentration of salicylate in the developing solution. The procedure also creates a uniform fliorescence background on the plate. After drying, the plate can be spotted with the sample solutions and developed as usual. The developing solution used contains $1.5 \times 10^{-2} \mathrm{M}$ sodium salicylate and $25 \%$ ethanol. The latter was included to avoid any hydrophobic interactions between the plate and the larger anion, salicylate. After developing for $8 \mathrm{~min}$, the fluorescence image can be viewed under a UV lamp after drying. The result is shown in Figure 1. clearly, the spots corresponding to each ion are visible even at the lowest concentration, despite an inhomogeneous background caused by the rough surface of the plate. It must be emphasized that the photographs shown here have essentially the same contrast as the images observed visually in a normally lit laboratory, i.e., the 


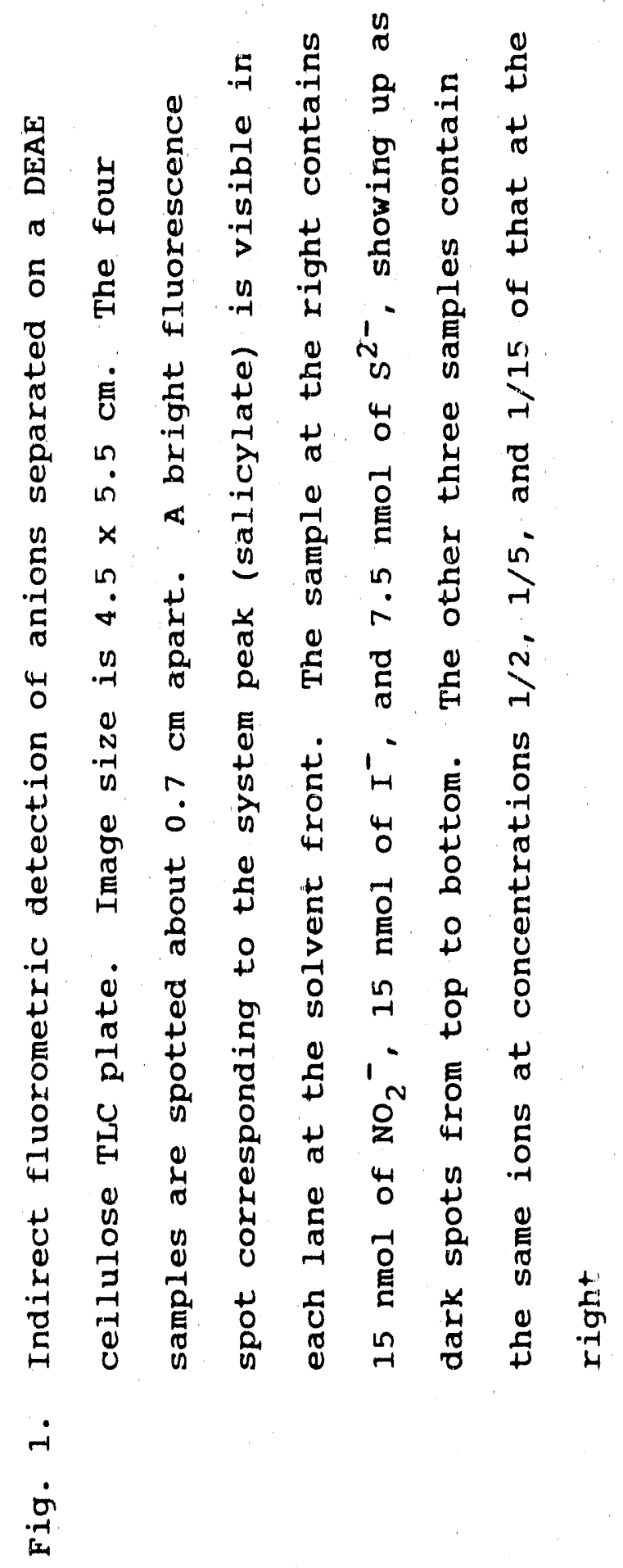




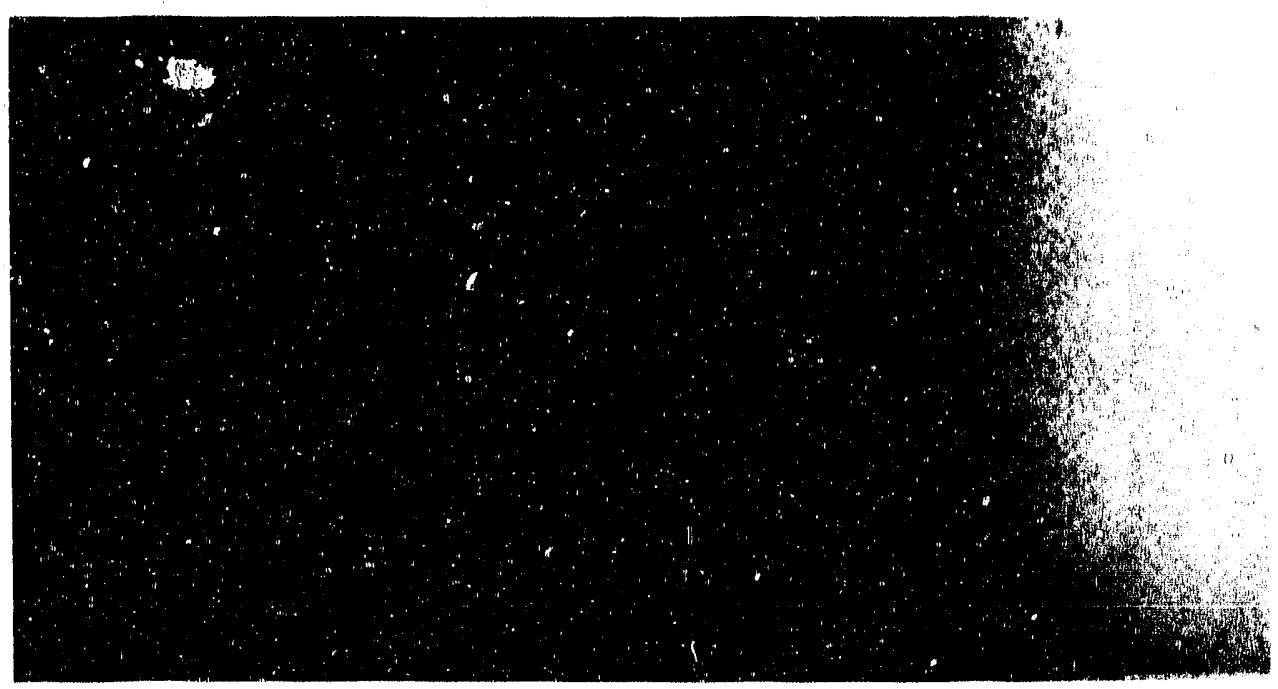


contrast has not been enhanced photographically. These anions do not absorb significantly at either the absorption or the emission wavelengths of salicylate. The nature of the response is one of replacement. Local charge neutrality (uniform cation distribution) and competition for available anion exchange sites cause a depletion of salicylate where the analyte ions reside, producing a lower fluorescence signal. At the solvent front in Figure 1, one can observe bright spots corresponaing to the "system peaks". The associated cations of the analyte are not retained, and they sweep along an equivalent amount of salicylate ions to where they reside.

The detection of the order of 10-100 ng of an anion in TLC is not particularly impressive, even though Figure 1 demonstrates the principle of indirect fluorescence detection. The optimization of detectability can be achieved following the corresponding scheme in IC $(13,14)$. In the indirect detection method, the dynamic reserve (which is defined as the ratio between the background signal and its noise level), the concentration of the visualization reagent, and the displacement ratio (which is defined in this work as the number of visualization ions which are transferred by one analyte ion) all play important roles in the sensitivity that can be achieved. 
The concentration detectability $\left(c_{1 i m}\right)$ at the detector is given by these parameters as follows:

$$
C_{1 \text { im }}=C_{m} / R D
$$

where $c_{m}$ is the concentration of the visualization reagent, $R$ is the displacement ratio, and $D$ is the dynamic reserve. The displacement ratio for monovalent ions is expected to be unity in ion-exchange chromatography (independent of the mobile phase concentration). So, one should decrease the concentration of the eluting ion, $c_{m}$. Ion-exchange stationary phases having very low capacities are required to demonstrate the separation of ions by use of a mobile phase with a low concentration of visualization reagent. The cellulose plates cannot be modified to have a lower capacity, so eluent concentrations below $10^{-2} \mathrm{M}$ salicylate cannot be used for separating these ions. We therefore dynamically modify silica gel plates with cetrimide to provide anion exchange sites, similar to a procedure reported for IC (14). From those experiments it may be concluded that cetrimide forms a double layer on the silica surface. The first layer of the reagent is formed by electrostatic interaction between nitrogen with a positive charge and a silanol group, while the secondary layer is formed by the hydrophobic interaction between the cetyl groups of the first layer and those of the reagent in the treating solution. The electrostatic interaction 
between the silica surface and the first layer is much stronger than the hydrophobic interaction between the first and second layer. Once treated, the cetrimide stays on the plate even though the subsequent solutions used do not contain cetrimide. By adjustment of the organic solvent fraction, the $\mathrm{pH}$, and the time of interaction, the amount of cetrimide (thus the capacity) on the TLC plate can be controlled.

Figure 2 shows the separation of anions on a dynamically modified TIC plate visualized by indirect fluorescence. The commercial TLC plate was treated with 10 $\mathrm{mM}$ cetrimide in water (pH 7.38 with $0.03 \mathrm{M}$ phosphate) for 2 h. After being rinsed with deionized water and dried, the plate was treated with a solution of $1 \times 10^{-4} \mathrm{M}$ sodium salicylate for $30 \mathrm{~min}$. After being rinsed and dried, the plate was spotted with samples and developed with $1 \times 10^{-3}$ M salicylate for $1.5 \mathrm{~min}$. Because of the low capacity of the plate, $\mathrm{NO}_{2}^{-}$(middle spot) begins to show tailing at the highest concentration tested. The results show that even $0.1 \mathrm{nmol}(4.6 \mathrm{ng})$ of $\mathrm{NO}_{2}^{-}$can be visually detected. This is an order of magnitude lower in concentration than the results obtained by using the cellulose plate. The developing solution is roughly an order of magnitude lower in salicylate concentration. The detectability found agrees well with the predictions of eq 1 . The fluorescence 
Fig. 2. Indirect florometric detection of anions separated on a dynamically (cetrimide) modified silica gel TLC plate. Image size is $4.5 \times 5.5 \mathrm{~cm}$. The four samples are spotted about $0.7 \mathrm{~cm}$ apart. The sampie at the right contains $10 \mathrm{nmol}$ of $\mathrm{IO}_{3}{ }^{-}, 5 \mathrm{nmol}$ of $\mathrm{NO}_{2}^{-}$, and $5 \mathrm{nmol}$ of $\mathrm{I}^{-}$, showing up as dark spots from top to bottom. The other three samples contain the same ions at concentration of $1 / 3$, $1 / 10$, and $1 / 30$ of that at the right 


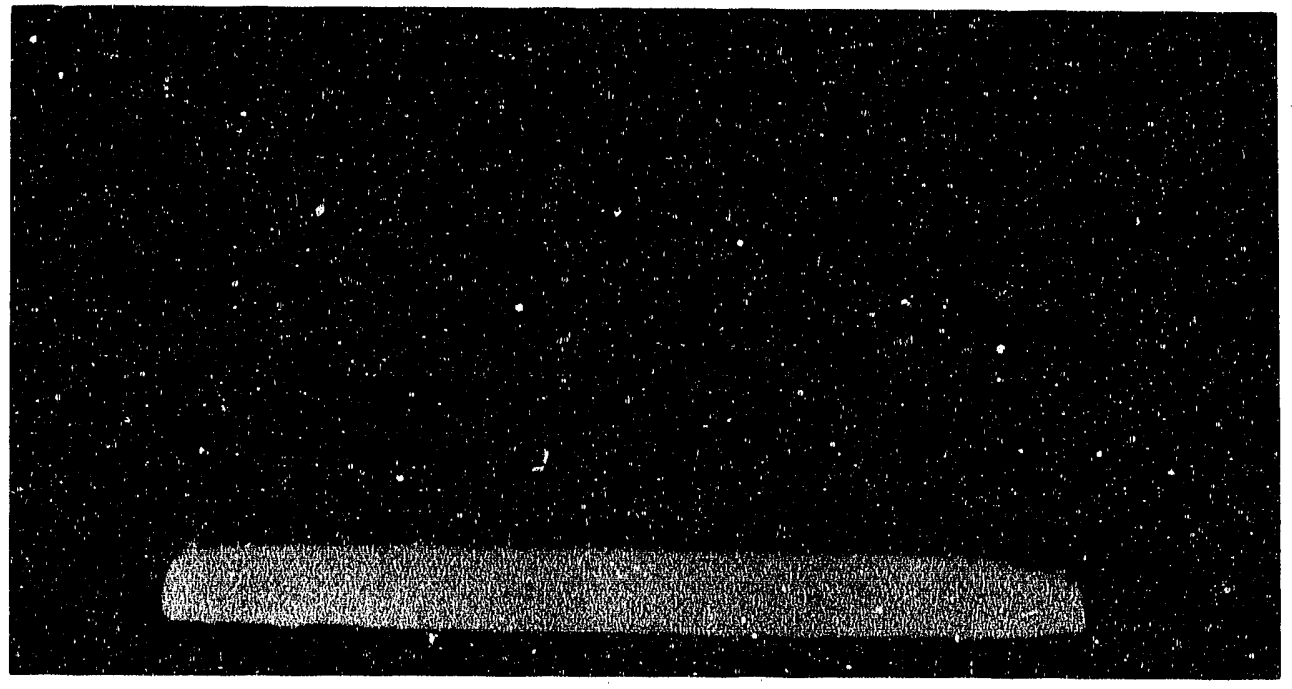


intensity created by $10^{-4}$ salicylate is marginal for visual observation, because the blue emission of salicylate is in a low sensitivity region of our eye. Thus we were unable to experiment with still lower salicylate concentrations to take advantage of eq 1 .

The results here are by no means fully optimized. Several improvements in detectability should be possible with further work. Visual observation is obviously poor compared to photuelectric recording (especially with signal averaging) with respect to $D$ in eq 1 . Fluorescence with high-frequency modulation should allow $D$ to improve (increase) by 2 to 3 orders of magnitude (10). The high sensitivity of laser-excited fluorescence should make it compatible with much lower salicylate concentrations, provided the natural fluorescence of the TLC substrate can be discriminated against. Multiple development (15) can effectively control the spread of the spots, so that the local concentration can be higher for the same quantity of analyte. One can also consider other schemes for dynamic modification to approach even lower capacities, analogous to adsorbing cetrimide on reversed-phase materials for IC (16).

In summary, we have presented a novel detection method for TLC based on indirect fluorescence. The demonstration system is chosen to elucidate the nature of the response 
and to establish the criteria for optimization, and not to specifically compete with IC for the same analytes. For example, other fluorescing anions can be used instead or salicylate to enhance separation and/or detection. Since detection is by charge displacement, any analyte anion should give a similar response. The method should also be applicable to the detection of cations (17) and even nonelectrolytes (13) on TLC plates. Finally, the pretreatment of the TLC plates here is necessitated by the lack of suitable commercial products. Those steps could very easily have been incorporated into the manufacturing process to allow essentially real-time monitoring of the separation process.

\section{References}

1. Fried, B.; Sherma, J. Thin-Layer Chromatography; Dekker: New York, 1986.

2. Giddings, J. C. Anal. Chem. 1984, 56, 1258A.

3. Gelderloos, D. G.; Rowlen, K. L.; Birks, J. W.; Avery, J. P.; Enke, C. G. Anal. Chem. 1986, 58, 900.

4. Fotiou, F. K.; Morris, M. D. Appl. Spectrosc. 1986, 40,700 .

5. Mcclelland, J. F. Anal. Chem. 1983, 55, 894.

6. Touchstone, J. C.; Dobblins, M. F. Practice of thin 
Layer Chromatography; Wiley: New York, 1983; pp. 235273.

7. Belenkii, B. G.; Gankina, E. S.; Adamovich, T. B.; Lobazov, A. Ph.; Nechaev, S. V.; Solonenko, M. G. I. Chromatogr. $1986, \underline{3} 65,315$.

8. Small, H.; Miller, T. E. Anal. Chem. 1982, 54, 462.

9. Bobbitt, D. R.; Yeung, E. S. Anal. Chem. 1984, 56, 1577 .

1.0. Mho, S. I.; Yeung, E. S. Anal. Chem. 1985, 57, 2253.

13. Pollack, V. J. Chromatogr. 1977, 133, 49.

12. Fritz, J. S.; Gierde, D. T.; Pohlandt, C. Ion Chromatography; Huthig: Heidelberg, 1982.

13. Takeuchi, T.; Yeung, E. S. I. Chromatogr. 1986, 366, 145.

14. Takeuchi, T.; Yeung, E. S. I. Chromatogr, 1986, 370, 83.

15. Tupille, T. H.; Perry, J. A. I. Chromatogr. 1974, 99, 231.

16. Pfeffer, W. D.; Takeuchi, T.; Yeung, E. S. Chromatographia $1987, \underline{24}, 123$.

17. Sherman, J. H.; Danielson, N. D. Anal. Chem. 1987, 59, 1483 . 
SECTION II

\title{
DETECTION OF CATIONS SEPARATED BY THIN-LAYER CHROMATOGRAPHY BY FLUORESCENCE QUENCHING OF ETHIDIUM BROMIDE
}

\section{Introduction}

\begin{abstract}
Recent advances in atomic spectroscopy have provided solutions to most of the major problems in elemental analysis. If speciation of the metallic ions is required, ion chromatography (1) can be used in conjunction with atomic spectroscopy or with conductivity detection. Despite these instrumental advances, there are still situations where thin layer chromatography (TLC) is the method of choice (2). TLC is easy for 2-dimensional separations. Multiple samples can be run on the same TLC plate. There are no problems with contamination or deterioration of performance, since a new stationary phase is used each time. TLC is suitable for highly retained analytes that create problems in column liquid chromatography (LC). Also, because of its simplicity, one can use TLC for optimizing conditions in LC.

One of the problems in TLC is detection. Other than for naturally absorbing analytes, some derivatization is
\end{abstract}


needed for visualization, typicaliy by using a spray reagent. In addition to difficulties associated with reliability and sensitivity, this is also a destructive detection method. There is therefore a need for alternative detection schemes for TLC, with desirable features such as being simple, sensitive, nondestructive, and rapid (for real-time monitoring).

Recently, we reported the detection of indirect fluorometric detection of anions (3) and nonelectrolytes (4) separated on TLC plates. There, displacement of a fluorescing additive in the developing solution by the analyte results in a lowering of the fluorescence intensity where the analyte resides. These are then observable as dark spots on a uniform fluorescence background. An analogous scheme is obviously possible for the detection of cations in TLC. This paper, however, reports a completely different mode of detection--one based on the quenching of fluorescence by the analyte. This is to be distinguished from the so-called fluorescence quenching detection (5) in earlier publications. We depend on a real change in the fluorescence quantum yield of the fluorophore, and not a simple depletion of the fluorescence intensity due to analyte absorption at the excitation or emission wavelengths. 
Experimental

Separations are done on $\mathrm{K}-6$ silica gel TLC plates (Whatman, Cli;ton, New Jersey, USA). To provide a uniform background fluorescence, the plates were immersed in a solution of $4 \times 10^{-5} \mathrm{M}$ ethidium bromide (Kodak, Rochester, New York, USA) at $\mathrm{pH}=3.8$ (phosphate buffer) for $2 \mathrm{~h}$. The plate was then dried and used. Mixtures of cations are spotted on the TLC plate using a microsyringe. The developing solution is $0.02 \mathrm{M}$ EDTA at $\mathrm{pH}=2.5$. Typical development time is $3 \mathrm{~min}$. After that, the plate is aried and viewed under a UV lamp (312 nm, Cole Palmer, Chicago, Illinois, USA, 9815).

Materials used are reagent grade chemicals without further purification. UV spectra are obtained on a conventional spectrophotometer (Shimadzu, Kyoto, Japan, UV240), and fluorescence spectra were obtained on a spectrofluorometer (Hitachi, Tokyo, Japan, Model 650-15).

\section{Results and Discussion.}

Fig. I shows the indirect fluorometric detection of several cations on a TLC plate. The photograph has not been enhanced in contrast, so that it shows the same level 


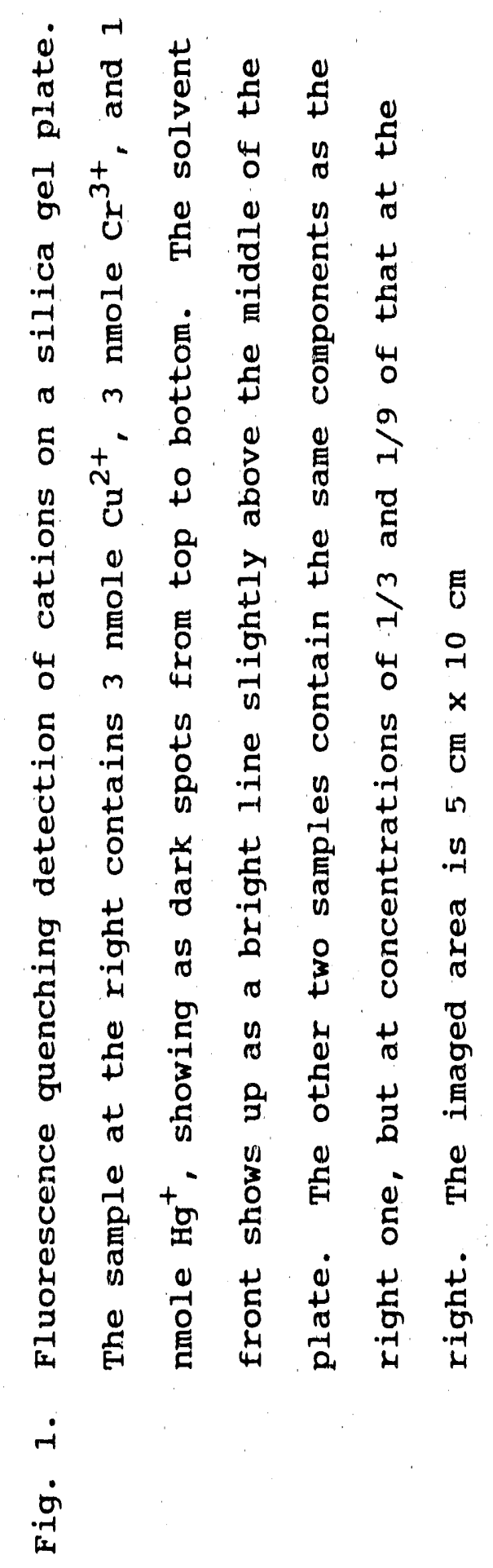




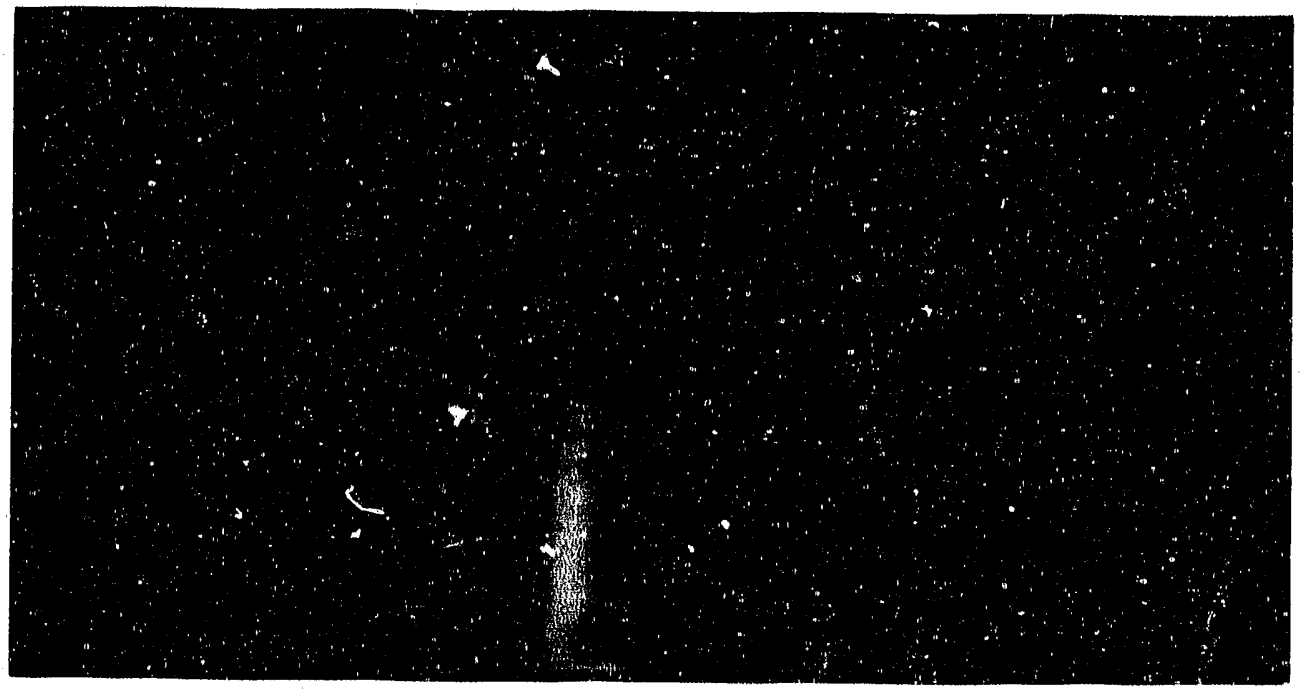


of contrast as seen by the eye. Subnanomole levels of each cation can be easily seen as dark spots superimposed on a bright orange fluorescence background on the plate. The identity of the individual ions is established by standards. The mechanism for the separation is by complexation with EDTA, and is well understood (6). The nature of the fluorescence response, however, is quite different from those reported previously. The structure of ethidium bromide is shown in Fig. 2. The interaction between the metal ions and ethidium results in quenching of the fluorescence (decrease in the fluorescence quantum yield). To our knowledge, this is the first observation of quenching of ethidium bromide fluorescence.

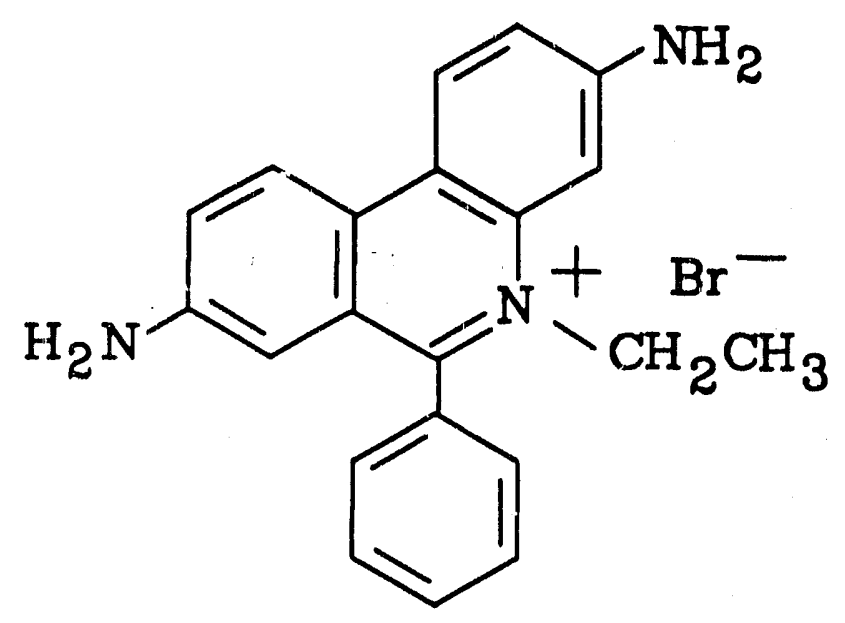

Fig. 2. Chemical structure of ethidium bromide 
To confirm that the signal is a result of fluorescence quenching, we have done some studies on TLC plates as well as in solution. First, solutions of the same ions were spotted on the TLC plate and dried without development. The same dark spots can be observed. There should be no displacement of ethidium bromide in this procedure, except for slight diffusion before the sample is dried. Clearly, this signal is quite different in nature from that in indirect fluorometry $(3,4)$. Second, since displacement is expected to be on a per charge basis, any cation should give a similar response. We surveyed a large number of cations, and found that no signal is produced when the cation is one of the following: $\mathrm{Na}^{+}, \mathrm{K}^{+}, \mathrm{Mg}^{2+}, \mathrm{Ba}^{2+}, \mathrm{Sr}^{2+}$, $\mathrm{Zn}^{2+}, \mathrm{Mn}^{2+}, \mathrm{Ca}^{2+}, \mathrm{Y}^{3+}, \mathrm{Ce}^{3+}, \mathrm{Se}^{3+}$, and $\mathrm{Sn}^{2+}$. Ions that only produce weak signals (faint spots) include $\mathrm{Al}^{3+}, \mathrm{Ni}^{2+}$, $\mathrm{CO}^{2+}$; and $\mathrm{Cd}^{2+}$. other ions show up as very dark spots; these include $\mathrm{Pb}^{2+}, \mathrm{As}^{3+}, \mathrm{Hg}^{+}, \mathrm{Hg}^{2+}, \mathrm{Cu}^{2+}, \mathrm{Ag}^{+}, \mathrm{Fe}^{3+}, \mathrm{Cr}^{3+}$, $\mathrm{Sb}^{3+}, \mathrm{Sn}^{4+}, \mathrm{Ti}^{3+}, \mathrm{V}^{3+}, \mathrm{V}^{4+}, \mathrm{Fe}^{2+}$, and $\mathrm{Ce}^{4+}$. Nonuniform response indicates specific interaction between the cation and ethidium bromide. Third, the magnitude of the response can be used to rule out light absorption by the cation at the excitation or the emission wavelength of ethidium bromide as a possible mechanism. Many of the cations, such as $\mathrm{Pb}^{2+}$, do absorb at $300 \mathrm{~nm}$, which is near the excitation wavelength. However, the peak molar absorptivity there is 
only $7.81 \mathrm{~mol}^{-1} \mathrm{~cm}^{-1}$. At the quantities used in Fig. 1 , there should not be any measurable depletion of the light from the UV lamp. Also, $\mathrm{Ni}^{2+}$ shows negligible absorption $\left(<11 \mathrm{~mol}^{-1} \mathrm{~cm}^{-1}\right)$ at $300 \mathrm{~nm}$ and $\mathrm{zn}^{2+}$ shows moderate absorption at $300 \mathrm{~nm}$. Yet, a response is observed for $\mathrm{Ni}^{2+}$ and no response is observed for $\mathrm{zn}^{2+}$. Fourth, there is evidence that electronic interaction occurs between some of these cations and ethidium bromide. A mixture of $1.2 \mathrm{x}$ $10^{-5} \mathrm{M}$ ethidium bromide and $0.1 \mathrm{M}$ of $\mathrm{Al}^{3+}$ produces an absorbance that is 1.4 times the sum of the absorbances of the individual solutions of the two components at $278 \mathrm{~nm}$. The structure of ethidium bromide does not suggest any special chelating or binding properties with respect to cations. However, the deviation from Beer's law for the mixture implies that there is probably analogous influence on the fluorescence quantum yield.

To confirm that a fluorescence quenching mechanism is responsible for the signal, we have measured the relative intensities of fluorescence for a $1.2 \times 10^{-5} \mathrm{M}$ solution of ethidium bromide in the presence of varying concentrations of $\operatorname{Cr}$ (III). The excitation wavelength was $278 \mathrm{~nm}$ and the emission wavelength was $600 \mathrm{~nm}$. A Stern-Volmer plot of the results is shown in Fig. 3. The ordinate is the reciprocal of the fluorescence intensity relative to that in the absence of $\operatorname{cr}($ III). A linear plot is obtained for this 



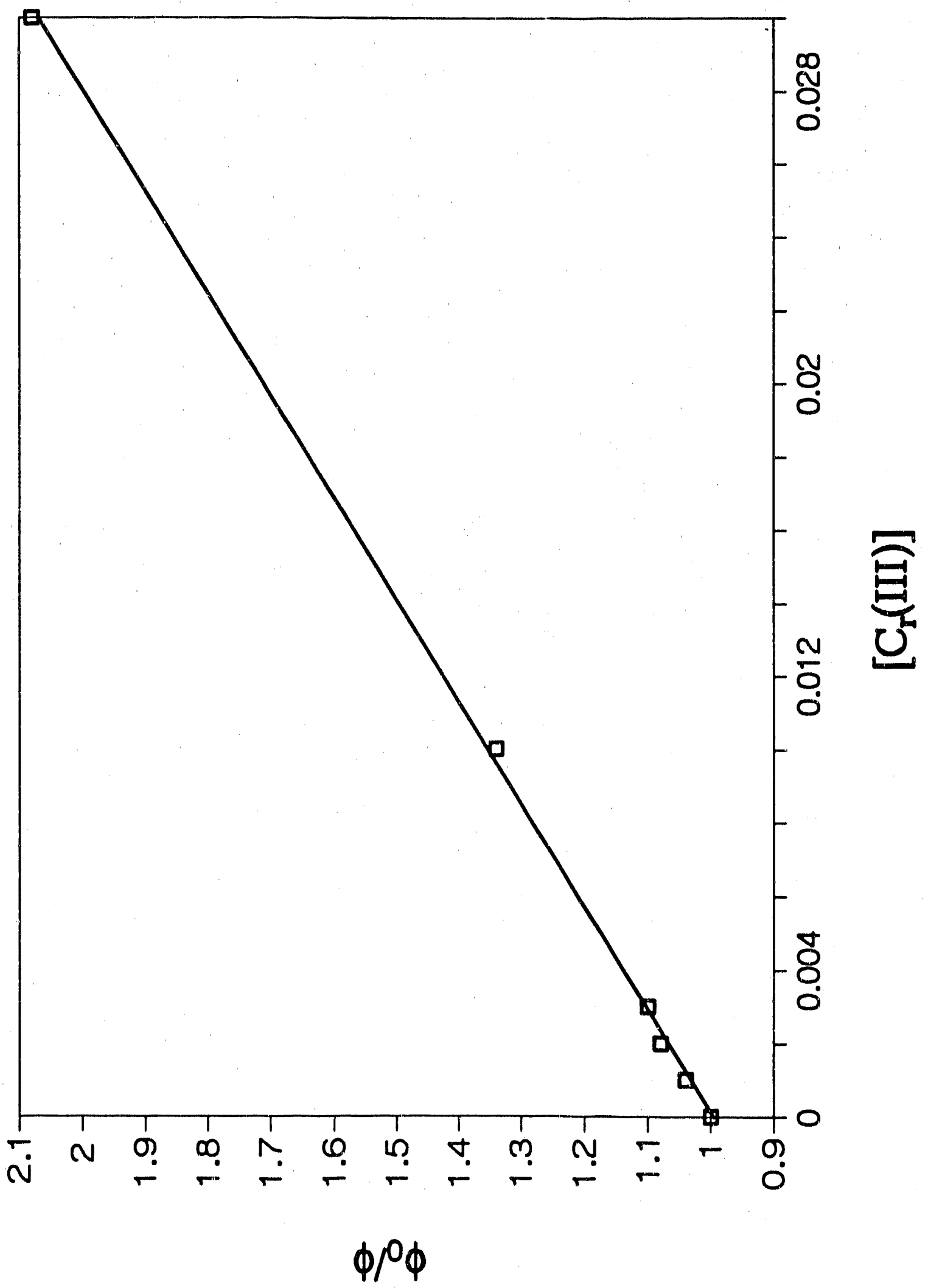


range, depicting typical quenching behavior. At higher $\mathrm{Cr}^{3+}$ concentrations, the plot becomes nonlinear, probably due to contributions from light absorption by $\mathrm{Cr}$ (III) at $278 \mathrm{~nm}$. Similar behavior is found for the other ions, when absorption by the ions themselves is properly accounted for. It is, however, difficult to compare the slopes for the various ions because of absorption differences. So, the magnitudes of the quenching interaction can only be qualitatively described.

Unlike the detection of ions by indirect fluorescence (3), the optimum conditions here are quite different. There is no need for substantially reducing the concentration of ethidium bromide. In general, quenching is much more efficient in reducing the fluorescence intensity than the one-to-one displacement process. Lower detection limits can thus be obtained. However, quenching depends on a specific interaction, and the detection scheme is no longer universal. Further, the fractional decrease in quantum yield should not depend on the concentration of the fluorophore at low concentrations. The absence of displacement here also means that detection is uncoupled from separation, and each can be optimized independently. Finally, quenching is in general a nonlinear process. Quantitation of the cations will require using many standards to establish the calibration curve. 
As before $(3,4)$, we should emphasize that the main advantage here is that the cations can be visualized without derivatization. In particular, ethidium bromide can be incorporated into the TLC plate during the manufacturing process, and real time detection is possible. The eye (and also the photographic plate) is not very suitable for distinguishing a dark spot on a bright background. A more instrumental approach (e.g., scanning the spot with a laser beam) should provide even better limits of detection.

\section{References}

1. Fritz, J. S.; Gierde, D. T.; Pohlandt, C. Ion Chromatography; Huthig: Heidelberg, 1982.

2. Fried, B.; Sherma, J. Thin Layer Chromatography; Dekker: New York, 1986.

3. Ma, Y.; Yeung, E. S. Anal. Chem. 1988, 60, 722 .

4. Ma, Y.; Yeung, E. S. I. Chromatogr. 1988, 455, 382.

5. Pollack, V. I. Chromatogr. 1977, 133, 49.

6. Varshney, K. G.; Khan, A. A.; Anwar, S. I. Liq. Chromatogr. 1985, 8, 1347. 
SECTION III

\section{INDIRECT FLUORIMETRIC DETECTION OF NONELECTROLYTES IN THIN-LAYER CHROMATOGRAPHY}

\section{Introduction}

Thin-layer chromatography (TLC) is one of the oldest and perhaps the simplest chromatographic separation techniques still in widespread use. In recent years, emphasis has been on high-performance liquid chromatography (HPLC) to separate complicated samples because of its high separation efficiency and advanced instrumental detection system. Therefore, TLC is far behind HPLC, even though a lot of developments in sorbent materials, particle sizes and detection instruments have been achieved (1-5) . However, TLC has certain advantages because it is an open bed while HPLC is a closed bed system. Since each plate is used only once, there is no possibility of poisoning a TLC plate, which is far too easy to do with an HPLC column. This may result in permanent damage to the column if samples cannot be removed. TLC plates, however, are free from this problem and highly retained components can still be detected. Simultaneous sample separation and detection (6) and two-dimensional separation (7-9) can be used. TLC 
is also quick, simple, convenient, inexpensive and disposable. TLC can complement HPLC as a pilot technique for the optimization of separation conditions (10) and can be used as a sample preparation technique for further separation $(11-12)$.

Detection in TLC, unlike HPLC, is a static process, being completely separated from chromatographic development.' So, a detector that works well for HPLC may not work for TLC. Detection of colored compounds, in general, causes no problem. The same holds true of compounds which exhibit fluorescence or phosphorescence under UV light. For colorless compounds, one usually adopts densitometry (13), directly if the compounds are UVabsorbing, or after color reaction (14) for which a specific spray reagent is required. Even though these methods are sometimes quite sensitive, they cannot be used to detect analytes which do not absorb in the UV region or have no appropriate spray reagents. The use of fluorescence layer (15-17) has been successfully demonstrated for detecting colorless compounds. However, this still requires that the analytes absorb either at the absorption or the emission wavelength of the fluorophore on the plate.

Recently, indirect fluorimetric detection of anions and nonelectrolytes in HPLC has been demonstrated (18-21). 
Briefly, a fluorophore in the mobile phase generates a large uniform background fluorescence. When the analytes elute, displacement of the fluorophore causes a change in the fluorescence intensity to allow detection. Following the same principles, the indirect fluorimetric detection of anions in TLC has also been achieved (22). This article will present an indirect fluorimetric detection method for nonelectrolytes (alkanols, bile acids and digoxin series) in TLC. Not only are detection limits improved, but the method is also much simpler and analysis time is much shorter compared with other detection methods in TLC for the same compounds $(23,24)$.

Experimental.

\section{Reagents}

HPLC grade acetonitrile and methanol, and certified grade methyl ethyl ketone were obtained from Fisher Scientific (Fairlawn, NJ, U.S.A.). HPLC grade chloroform was obtained from Burdick \& Jackson Labs. (Muskegon, MI, U.S.A.). HPLC grade tetrahydrofuran and analytical grade salicylic acid were obtained from Baker (Phillipsburg, NJ, U.S.A.). Alkanols, digoxin, digitoxin, taurocholic acid and taurodeoxycholic acid were purchased from Aldrich (Milwaukee, WI, U.S.A.). Digoxigenin, glycocholic acid and 
glycodeoxycholic acid were purchased from Sigma (st. Louis, MO, U.S.A.) . 2-(1,1'-Bipheny 1$)-4-y I-5-$ phenyl-1,3,4oxadiazole (PED) was purchased from Eastman-Kodak. (Rochester, NY, U.S.A.) . 2-(1-Naphthalenyl)-5phenyloxazale from Lambda Physik (Acton, MA, U.S.A.) was used to prepare pretreating solutions or eluents.

\section{Materials}

$\mathrm{K}_{6}$ silica gel plates and $\mathrm{KC}_{18}$ reversed-phase plates were purchased from whatman chemical Separation (Clifton, NJ, U.S.A.). Adsorbosil RP-HPTLC plates were obtained from Alltech Assoc. (Deerfield, IL, U.S.A.). UV lamps (312 nm, $254 \mathrm{~nm}$ ) were purchased from Cole Palmer (Chicago, IL, U.S.A.). A microsyringe calibrated to $0.1 \mu l$ units was purchased from Hamilton (Reno, NV, U.S.A.).

\section{Sample preparations}

Alcohol mixtures were prepared by dissolving decanol, dodecanol, tetradecanol and haxadecanol in acetonitriletoluene $(90: 10)$. Bile acids mixtures were prepared by dissolving taurocholic acid (TC), taurodeoxycholic acid (TCD), glycocholic acid (GC), glycodeoxycholic acid (GDC) in methanol. Digoxin series mixtures were prepared by dissolving digoxin, digitoxin and digoxigenin in chloroform-methanol (2:1). 
Chromatography and detection

Alcohols (a) The Adsorbosil RP-HPTLC plate is immersed in acetonitrile-water (40:60) containing $2 x$ $10^{-5} \mathrm{M} P B D$ for $2 \mathrm{~h}$. The plate is then dried with a heatgun. Samples are spotted by a micro-syringe. The plate was developed by acetonitrile-water (98:2) containing $4 \times$ $10^{-3} \mathrm{M}$ PBD for $6 \mathrm{~min}$. After drying the plate again, the plate was placed under a UV lamp $(312 \mathrm{~nm})$. A series of negative spots can be observed.

(b) The samples were spotted on a $\mathrm{KC}_{18}$ reversedphase plate directly (no pretreatment) by a microsyringe and the plate was developed by acetonitrile-water (98:2) containing $4 \times 10^{-5} \mathrm{M} \alpha-\mathrm{NPO}$ for $6 \mathrm{~min}$. The plate is then dried and visualized under the UV lamp (3.12 nm). A series of positive spots can be observed.

\section{Bile acids $\quad A K_{6}$ silica gel plate was treated by} chloroform-acetonitrile $(50: 50)$ containing $1 \times 10^{-4} \mathrm{M}$ salicylic acid for $1 \mathrm{~h}$ and was then dried. After spotting the bile acid mixture on the plate by a microsyringe, the plate is developed with chloroform-methanol-methyl ethyl ketone (46:46:8) containing $3 \times 10^{-3} \mathrm{M}$ salicylic acid for $24 \mathrm{~min}$. The plate was then dried and visualized under a UV lamp (312 nm). A series of positive spots can be observed. 
Digoxin series (a) An Adsorbosil RP-HPTLC plate was pretreated with acetonitrile-water $(40: 60)$ containing 1 $\times 10^{-5} \mathrm{M} \alpha$-NPO for $1 \mathrm{~h}$ and was dried with a heat-gun. Samples were spotted with a microsyringe, and the plate was developed by methanol-water (7:3) containing $4 \times 10^{-5} \mathrm{M} \alpha-$ NPO for $8 \mathrm{~min}$. The plate was then dried and visualized under a UV lamp $(254 \mathrm{~nm})$. A series of negative spots can be observed.

(b) After spotting the samples o.7 a $\mathrm{KC}_{18} \mathrm{RP}-\mathrm{TLC}$ plate directly (no pretreatment) by a microsyringe, the plate is developed by methanol-water $(7: 3)$ containing $2 x$ $10^{-3} \mathrm{M}$ salicylic acid for $8 \mathrm{~min}$. Then, the plate is dried and visualized under a UV lamp $(312 \mathrm{~nm})$. A series of positive spots can be observed.

\section{Results and Discussion}

Figs. 1 and 2 demonstrate the indirect fluorometric detection of alkanols by reversed-phase TLC. Nanomole levels of each alkanol can be detected by our eyes. The dark spots in Fig. 1 are not due to the presence of an absorbing species. Rather, less of the fluorophore exists locally because it has been transferred out of the stationary phase by the analyte. It should be mentioned that the positive (higher intensity) spots observed in 
61

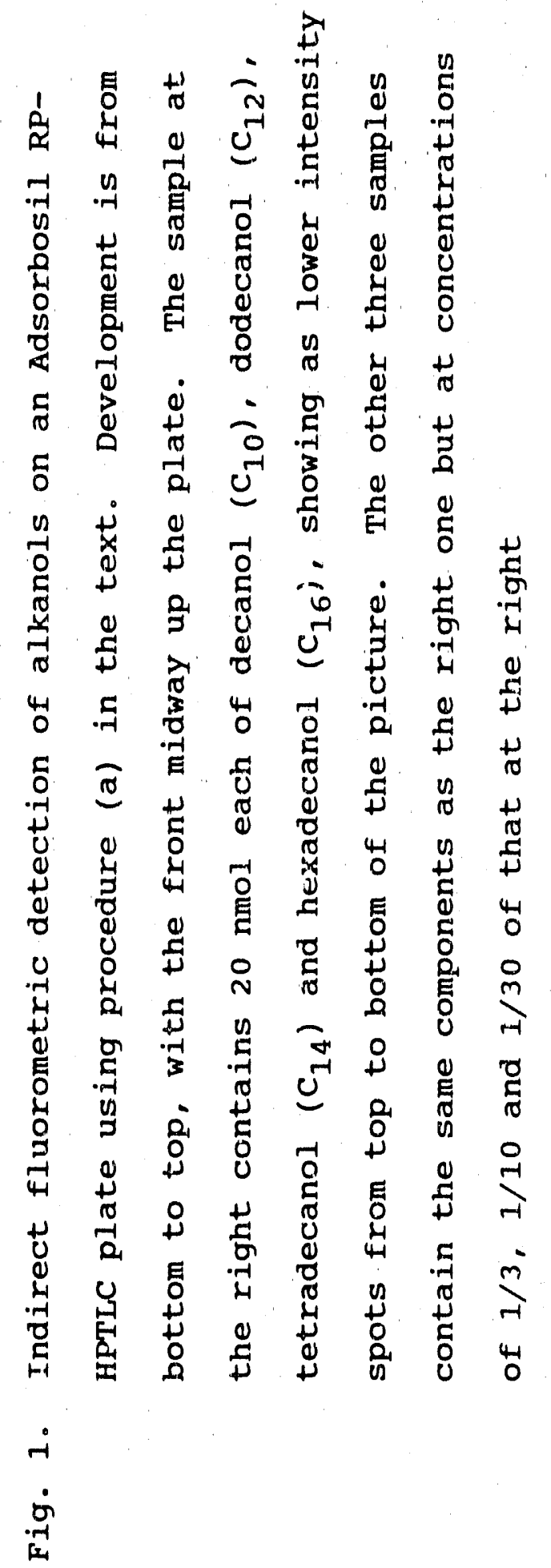




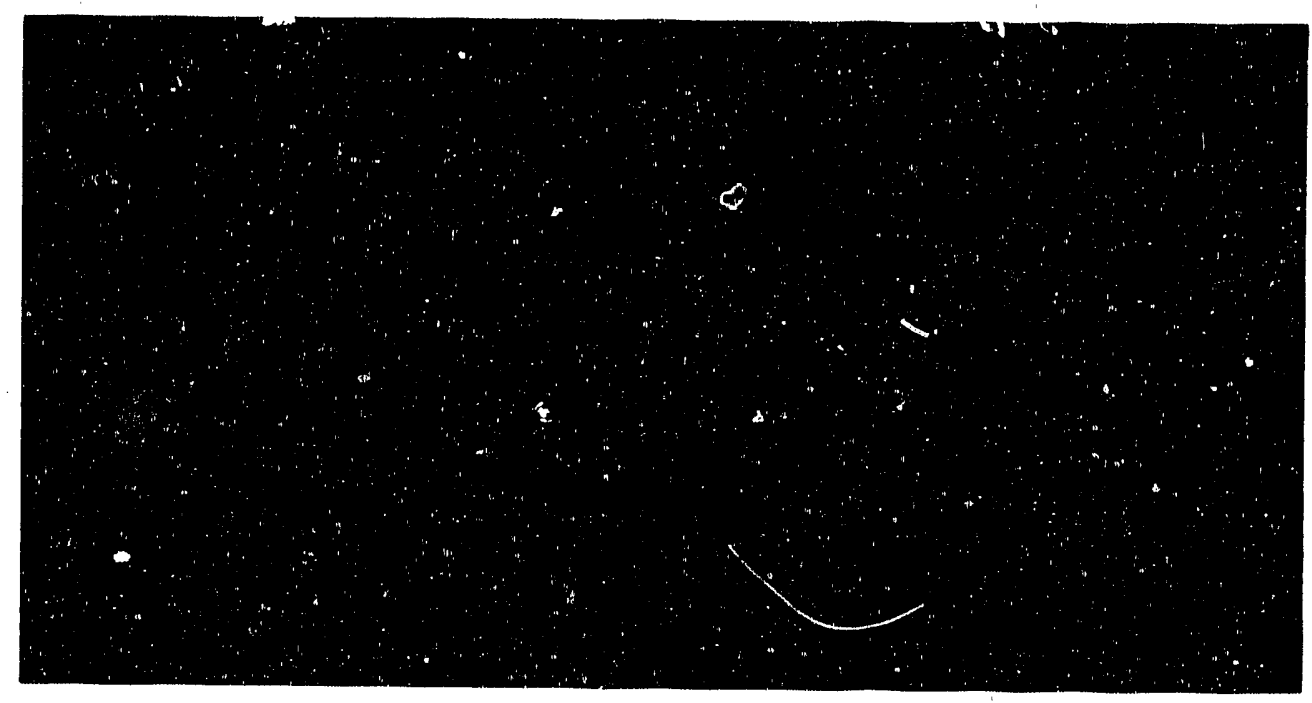




\section{3}

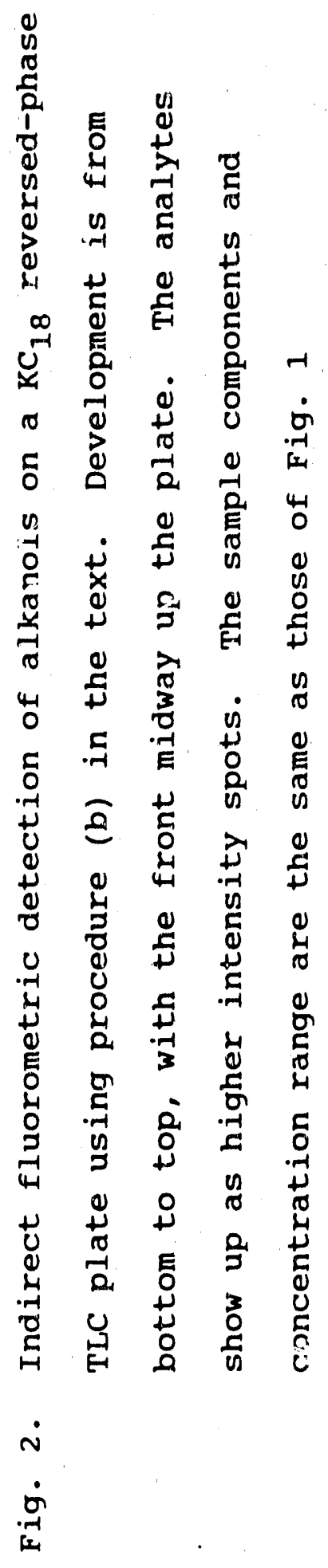




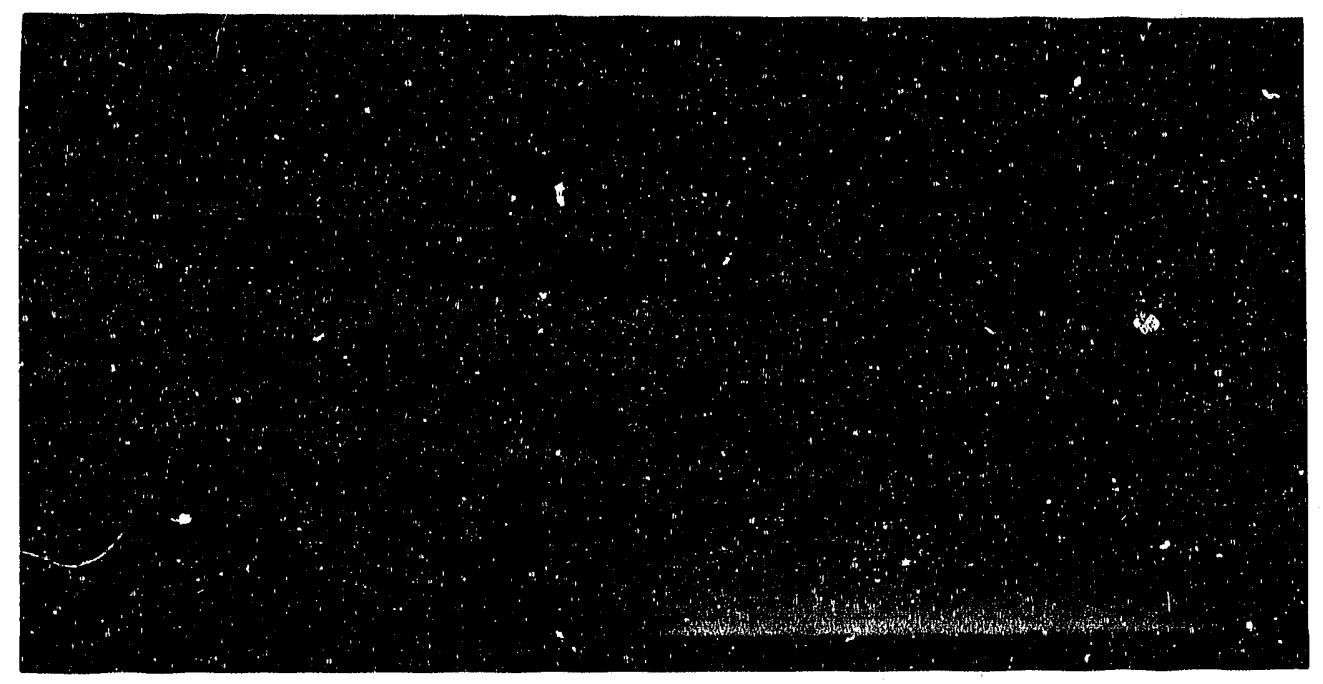


Fig. 2 are not due to the fluorescence of alkanols. The reason for this phenomenon is that alkanols transfer visualizing reagent ( $\alpha$-NPO here) from the mobile phase to the stationary phase. So, where the sample spot is, more of the fluorophore exists, and a stronger fluorescence signal is observed. An earlier paper (25) had a detailed discussion on this. The choice of fluorophore determines whether positive or negative (lower intensity) spots are observed, even though the same developing solution is used. This clearly shows that differences in partition into the stationary phase between the fluorophore and the analytes is responsible for the signal.

Fig. 3 shows the indirect fluorometric detection of bile acids. Subnanomoles of each can be detected. The reason for the presence of positive spots is the same as that for alkanols. Touchstone et al. (24) had previously demonstrated the separation of conjugated bile acids on reversed-phase TLC and detection by a spectrodensitometer in the transmission mode. But the method is time consuming and one needs to search for a specific spray reagent. By our approach, separation is faster and detection is simpler. Similar detection limits can be achieved even by our naked eye. With a scanning laser beam for excitation, one would expect even better detection due to signal averaging and intensity enhancement. 
66

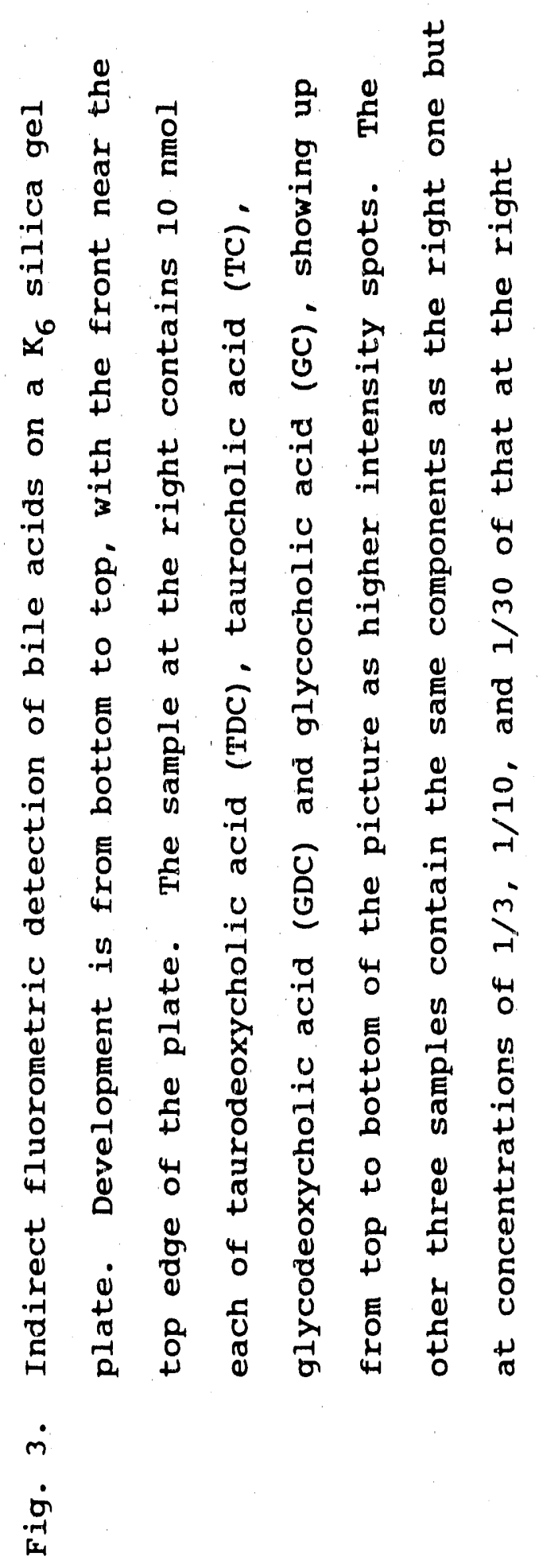




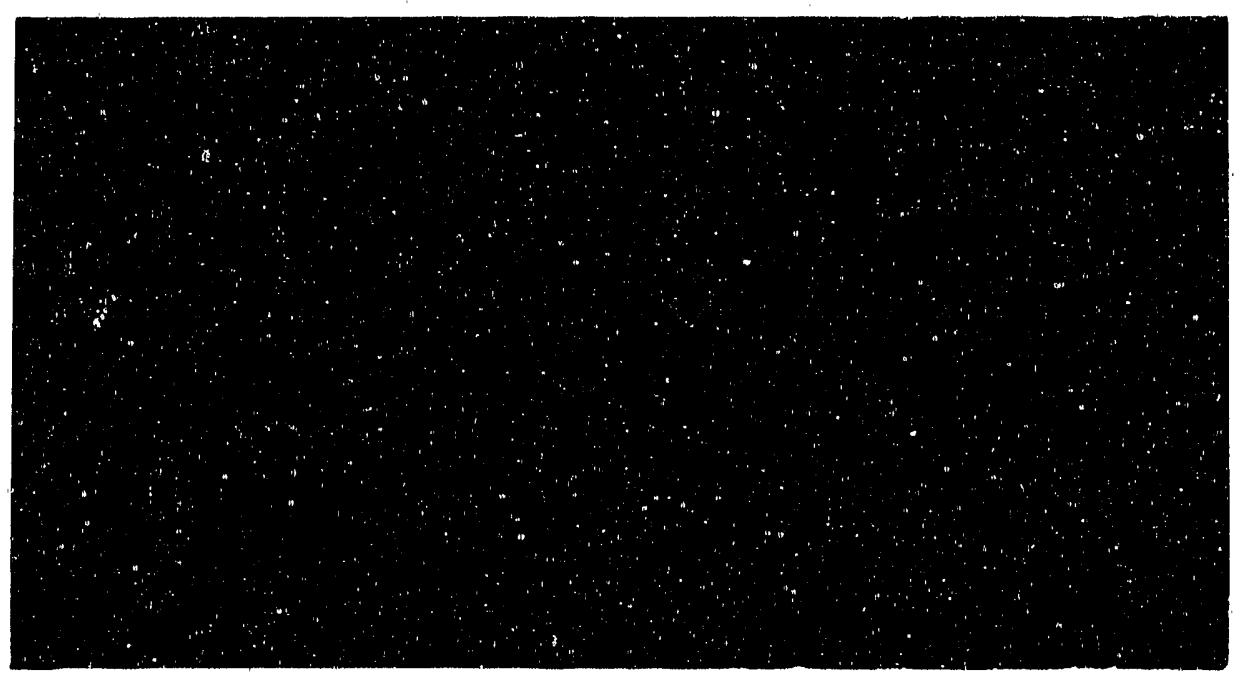




\section{$6^{9}$}

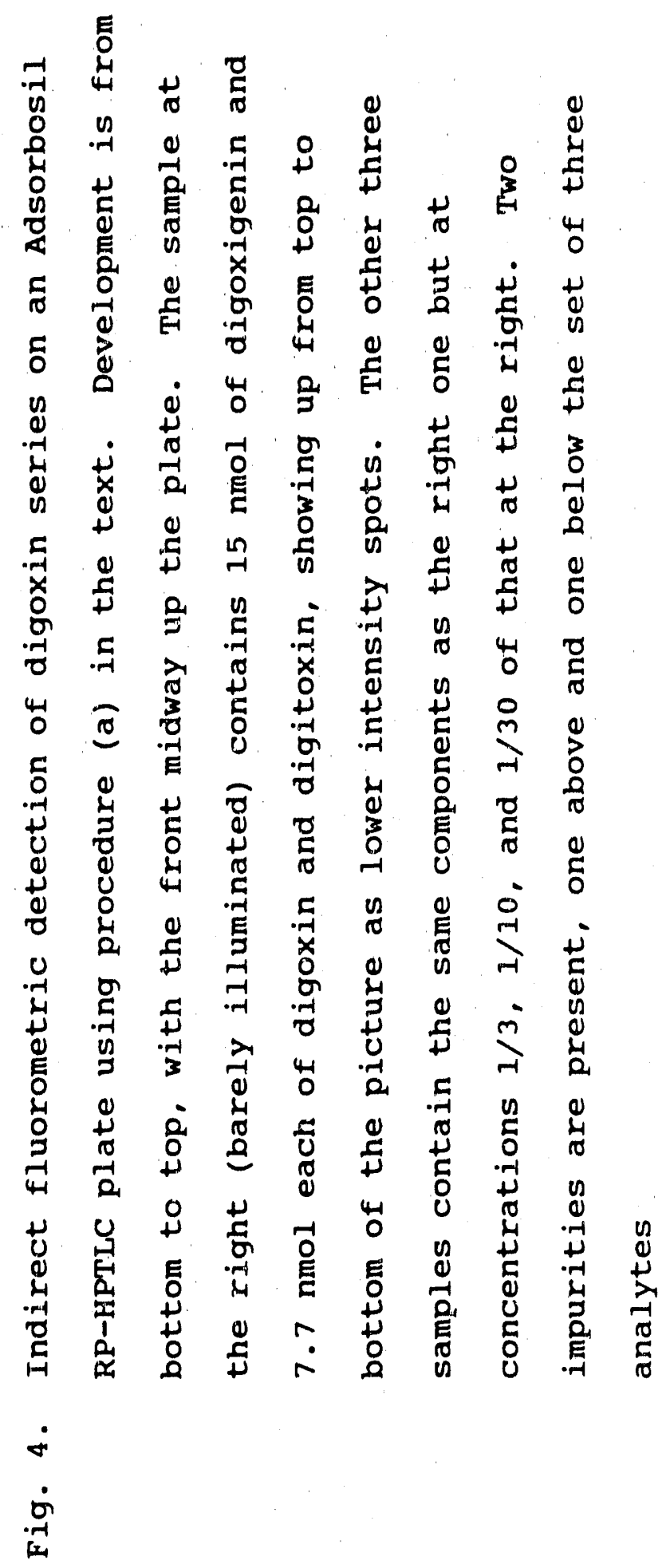




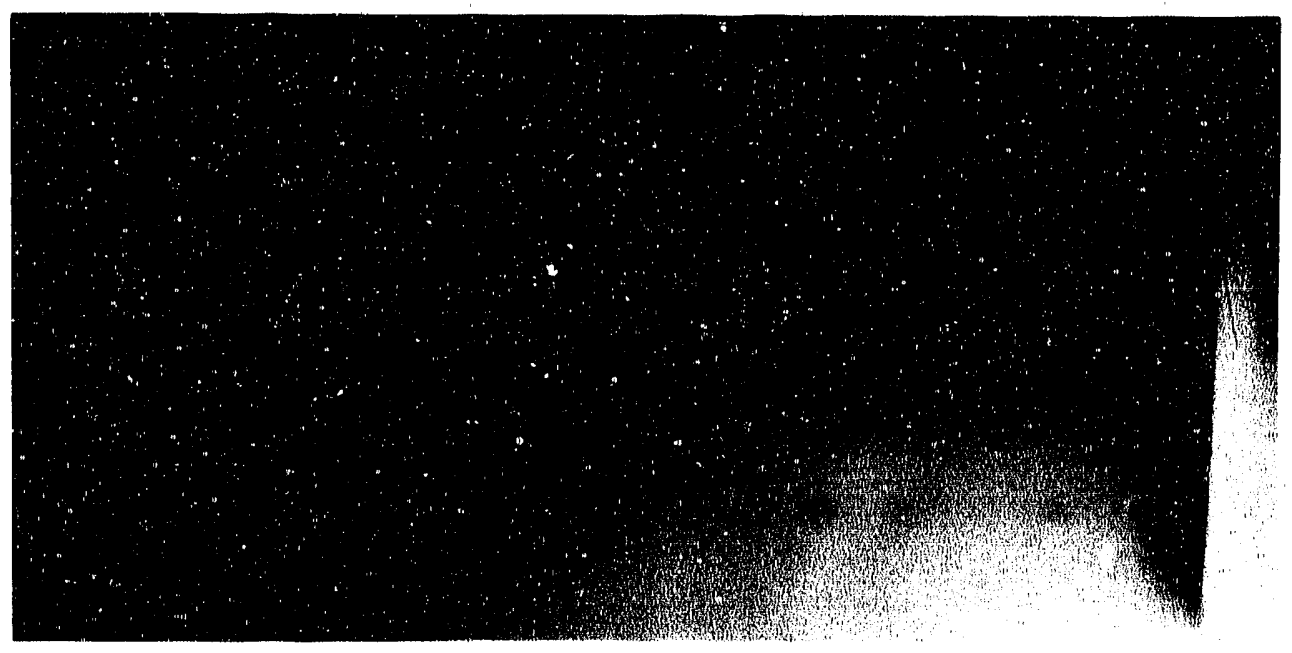


71

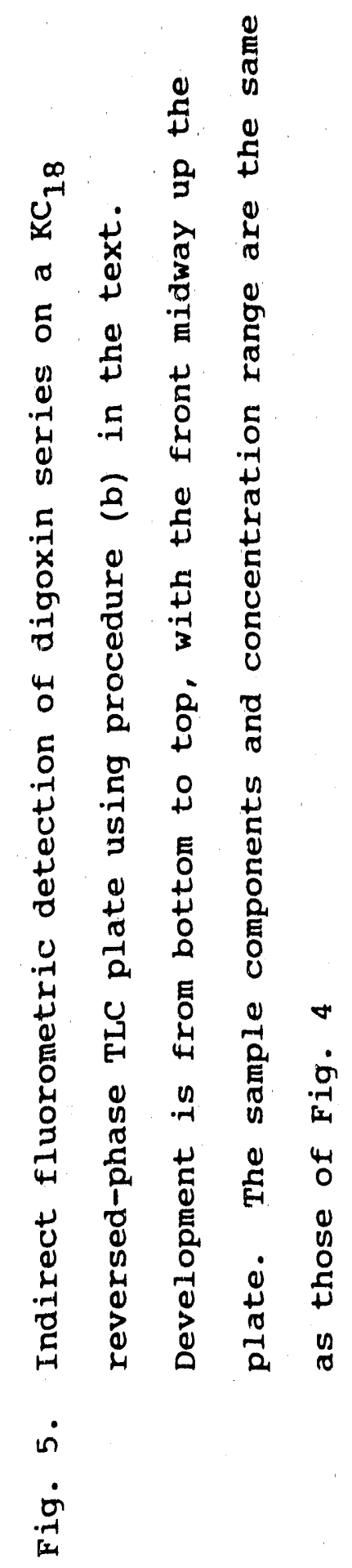




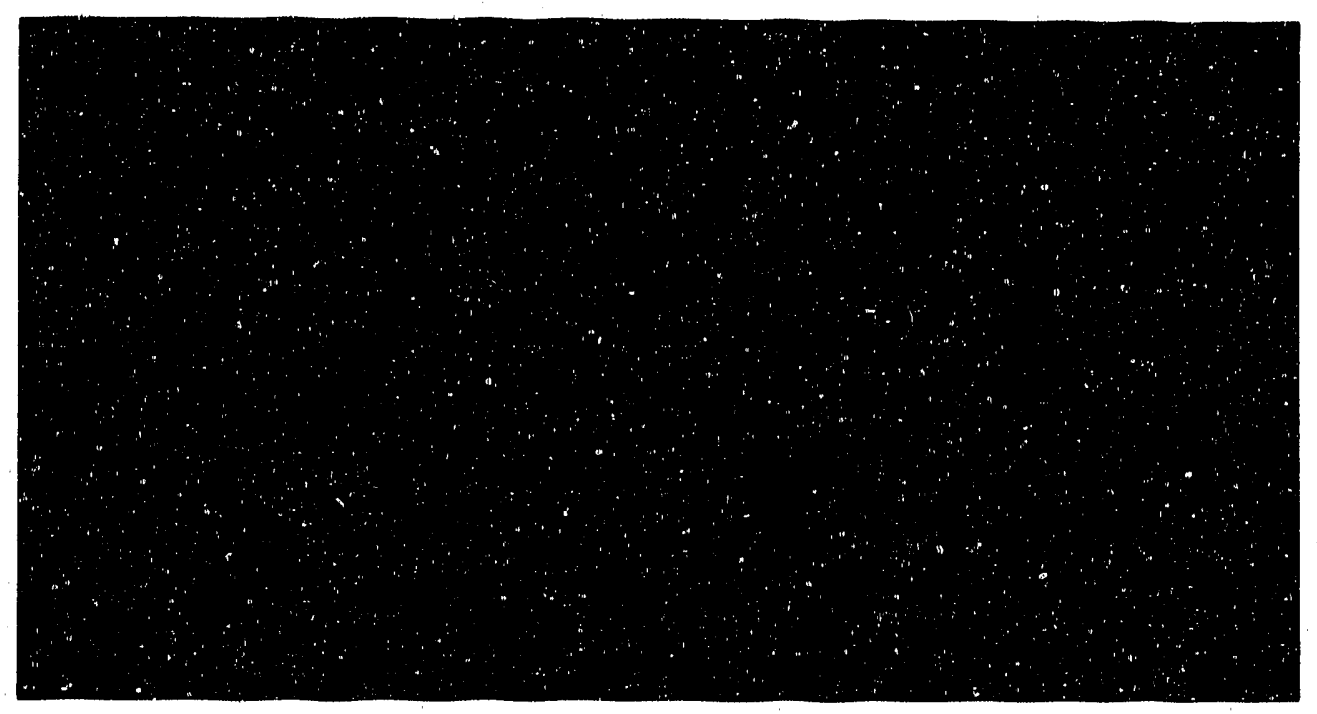


In order to improve the detection limit or sensitivity of indirect fluorometric detection further in TLC, the background signal should be decreased as much as possible. This can be achieved by decreasing the concentration of the visualization reagent in the pretreating solution or in the developing solution. However, if the background signal is too low, the displacement ratio may also decrease and contrast will be lost.

The visual (or photographic) observation in this work is obviously poor compared to instrumental detection methods with respect to $D_{R}$ in eq. 1. Therefore, further improvements in detectability should be possible. Fluorescence with high-frequency modulation should allow $D_{R}$ to increase by 2 to 3 orders of magnitude (18). The high sensitivity of laser-excited fluorescence should make it compatible with much lower fluorophore concentrations, if the natural fluorescence of the TLC substrate can be discriminated against.

Conclusion

Indirect fluorometric detection of nonelectrolytes (alkanols, bile acids and digoxin series) has been presented. Nanomolar detection limits were achieved. The selection of the visualization reagent and the 
chromatographic conditions is important because of the dependence of displacement ratio and spot character (negative spot or positive spot) on it. With minor modifications of the recipes given in this work, one can now essentially combine indirect fluorometric detection with any TLC separation.

\section{References}

1. Belchamber, R. M.; Read, H.; Roberts, J. D. M. I. Chromatogr. $1987,395,47$.

2. Burns, D. H.; Callis, J. B.; Christian, G. D. Trends Anal. Chem. $1986,5,50$.

3. Fried, B. ; Sherma, J. Thin Layer Chromatography:

Dekker: New York, 1986.

4. Donovan, J.; Gould, M. ; Majors, R. LC-GC Mag. 1987, $\underline{5}$, 1024.

5. Belenkii, B. G.; Gankina, E. S.; Adamovich, T. B.; Lobazov, A. P.; Nechaev S. V.; Solonento, M. G. I. Chromatogr. $1986, \underline{365}, 315$.

6. Gelderloose, D. G.; Rowlen, K. L.; Birks, J.W.; Avery, J. P.; Enke, C. G. Anal. Chem. 1986, 58, 900.

7. Despiegeleer, B.; Van den Bossche, W. : DeMoerloose, P.: Massart, D. Chromatographia 1987, 23, 407.

8. Matysik, G.; Soczewinski, E. I. Chromatogr. 1986, 
$369,19$.

9. Giddings, J. C. Anal. Chem. 1984, 56, 1258 A.

10. Jost, W.; Hauck, H. E.; Eisenbeiss, F. Kontakte (Darmstadt) $1984, \underline{3}, 45$.

11. White, R. L. Anal. Chem. 1985, 57, 1819.

12. Shafer, K. H.; Herman, J. A.; Bui, H. Am. Lab. (Fairfield, Conn.) 1988, February, 142.

13. Touchstone J. C.; Sherma, J. Densitometry in Thin Layer Chromatography; Wiley: New York, 1979.

14. Kirchner, J. G. Thin-Layer Chromatography; Wiley: New York, $2.978 ;$ p. 193.

15. Sease, J. W. I. Am. Chem. Soc. 1947, 69, 2242 .

16. Kirchner, J. G.; Miller, J. M.; Keller, G. J. Anal. Chem. 1951, 23, 420 .

17. Miller, J. M.; Kirchner, J. G. Anal. Chem. 1954, 26, 2002 .

18. Mho, S.; Yeung, E. S. Anal. Chem. 1985, 57, 2253.

19. Takeuchi, T.; Yeung, E. S. I. Chromatogr. 1986, 370, 83.

20. Takeuchi, T.; Yeung, E. S. I. Chromatogr. 1986, 366, 145 .

21. Pfeffer, W.; Takeuchi, T; Yeung, E. S. Chromatographia $1987, \underline{24}, 123$.

22. Ma, Y.; Yeung, E. S. Anal. Chem. 1988, 60, 722 . 23. Bloch, D. E. I. Assoc. Off. Anal. Chem. 1980, 63, 
707.

24. Touchstone, J. C.; Levitt, R. E.; Levin, S. S.;

Soloway, R. D. Lipids 1980, 15, 386.

25. Stranahan, J. J.; Deming, S. N. Anal. Chem. 1982, 54, 1540 .

26. Takeuchi, T.; Ishii, D. I. Chromatogr. 1987, 396, 149 . 
SECTTON IV

DETERMINATION OF COMPONENTS IN BEVERAGES

BY THIN-IAYYER CHROMATOGRAPHY --

AN UNDERGRADUATE ANALYTICAL CHEM:LSTRY EXPERIMENT

Introduction

A variety of experiments in righ performance liquid chromatography (HPLC) have been proposed for the undergraduate laboratory since the late 1970s (1-8). A popular one used in the undergraduate instrumental analysis laboratory is the determination of caffeine, saccharin and sodium benzoate in beverages by reversed-phase HPLC (7, 8). Thin-layer chromatography (TLC) is also applicable to this separation. However, this type of experiment (9) seems to be ignored in the undergraduate analytical laboratory. The reason may be due to difficulties in detection once the components are separated. Normally, one is limited to mixtures of dyes which bear little resemblance to "real samples".

From a pedagogical point of view, a properly designed TLC laboratory experiment lias many advantages. Student? can visualize the actlial separation of the components from each other in real time. The phenomenon of bar. broadening 
can be observed directly. The principles of twodimensional separation can be introduced. Contamination in between experiments is 1 iminated entirely. Differest detection modes can be incorporated simultaneously.

From a practical point of view, TLC is also superior to HPLC. The initial setup cost is substantially lower. The maintenance costs for pumps and detectors are nonexistent. The operating costs (consumption of solvents and stationary phases) are also much less compared to the corresponding HPLC experiments.

In this paper, we describe a simple and interesting TLC experiment using three different fluorescence detection principles for the determination of caffeine, saccharin and sodium benzoate in beverages. The total analysis time is less than three hours. The level of sophistication of the experiment can be readily tailored to fit a particular group of students.

Experimental

Reagents used are HPLC grade acetonitrile, certified grade methyl ethyl ketone, amnonium acetate, sodium benzoate, caffeine, saccharin, Nile Blue A perchlorate, and glacial acetic acid. Deionized water was used after further purification on a Milli-Q system. 
Materials used include Adsorbsil RP HPTLC plates (10 x $20 \mathrm{~cm}$, prescored), two UV lamps (254 nm, $312 \mathrm{~nm})$, and a microsyringe calibrated to $0.1 \mu \mathrm{L}$ units.

\section{Procedure}

(a) stock Nile Blue solution. Weigh $50 \mathrm{mg} \mathrm{Nile}$ Blue into a $50 \mathrm{ml}$ volumetric flask. Dissolve and dilute to the mark with acetonitrile. The concentration will be $1 \mathrm{mg} / \mathrm{ml}\left(i . e ., 2.4 \times 10^{-3} \mathrm{M}\right)$.

(b) Pretreatment solution Pipet $2.5 \mathrm{ml}$ stock Nile Blue solution into an $800 \mathrm{ml}$ beaker, then dilute to $600 \mathrm{ml}$ with acetonitrile and stir the solution to become homogenous. The concentration will be $1 \times 10^{-5} \mathrm{M}$.

(c) Pretreatmert of plates Break a TLC plate into 4 small ones $(5 \times 10 \mathrm{~cm})$. Immerse 3 of them into the pretreatment solution for 30 minutes. Then take them out with a pair of tweezers and dry them with a heat gun.

(d) standard and sample solutions During the pretreatment of TIC plates, standard (saccharin, caffeine and sodium benzoate) solutions and the sample solution can be prepared. Dissolve $20 \mathrm{mg}$ each of saccharin, caffeine and sodium benzoate in methanol in three separate 10-ml volumetric flasks and dilute to the mark. The concentration of each is $2 \mathrm{mg} / \mathrm{ml}$. Next, dissolve $20 \mathrm{mg}$ of saccharin, caffeine ana socium benzoate into one 10-ml 
flask with methanol and dilute to the mark as a mixed standard solution. The concentration is also $2 \mathrm{mg} / \mathrm{ml}$ for each component. Then pipet $5 \mathrm{ml}$ and $2 \mathrm{ml}$ of the above standard mixture solution into two $10-\mathrm{ml}$ volumetric flasks separately and dilute to the mark with methanol. The concentrations are $1 \mathrm{mg} / \mathrm{ml}$ and $0.4 \mathrm{mg} / \mathrm{ml}$ for each component, respectively. The sample solution can be easily prepared by pipeting $1 \mathrm{ml}$ of soft drink and $1 \mathrm{ml}$ methanol into a $3 \mathrm{ml}$ vial and mixing thoroughly (in this report, we used diet Shasta as a sample).

(e) Developing solution The developing solution used in this experiment contains $1 \times 10^{-4}$ M Nile Blue in $22 \%$ acetonitrile, $28 \%$ methyl ethyl ketone and $50 \%$ aqueous solution containing $0.6 \%(\mathrm{w} / \mathrm{v})$ ammonium acetate and $2.4 \%$ (v/v) glacial acetic acid.

(f) Spotting of standards, development and visualization pake one of the pretreated plates, spot separately $1 \mu \mathrm{L}$ standard saccharin, caffeine and sodium benzoate solutions $1 \mathrm{~cm}$ apart on the plate $1 \mathrm{~cm}$ from the bottom. Here it should be noted that in order to get a good spot shape, the 1 ml solution should be spotted gradually (e.g., $0.1 \mu l$ each time). If done properly, the original spots are very small (spot diameter is about 1 $\mathrm{mm})$. Then the plate is developed in an upward direction for 6 minutes. After drying the plate with a heat gun, it 
can be visualized under a UV lamp in a relatively dark room. Three spots at different heights should be observed. Students should measure the migration distance of the solvent front and each of the spots. $R_{f}$ value for each standard sample should be calculated based on the equation:

$$
R_{f}=\frac{\text { migration distance of the sample spot }}{\text { migration distance of the solvent front }}
$$

(g) Identification of each component in the standard mixture spot $1 \mu 1$ solutions for each of the three standard mixtures on another pretreated plate following the same procedure as step (f). After development, each component can be identified based on the $R_{f}$ values calculated in step (f). Also, different intensities should be observed for different concentrations of the samples.

(h) Semi-quantitative determination of saccharin, caffeine and sodium benzoate in soft drinks spot $6 \mu \mathrm{l}$ of the sample solution and $1 \mu l$ each of three standara mixture solutions separately on the third pretreated plate. The sample solution should also be spotted gradually (e.g., $0.4 \mu l$ each time) to obtain a small initial spot. After development, the plate can be visualized under a UV lamp. The amount of saccharin, caffeine and benzoate can be determined by comparing the 
intensities of the sample component spots with those of the standards.

Results and Discussion

Figure 1 demonstrates the separation and the fluorometric detection of saccharin, caffeine and benzoate in mixtures of standards (right) and in a soft-drink sample (left). All the spots can be seen clearly. Several important concepts can be learned from such an experiment. 1. Detection by fluorescence is illustrated by the spots corresponding to saccharin. Under normal room light, the spot cannot be seen. Excitation by UV light (254 $\mathrm{nm}$ or $312 \mathrm{~nm}$ ) causes re-emission to give a bright spot. In fact, an unidentified component in this soft drink at the solvent front also shows fluorescence. The intensity of fluorescence depends on concentration (standard solutions).

2. Detection by absorption is illustrated by the spots corresponding to caffeine and benzoate. The TLC plate treated in this manner gives a strong background fluorescence when irradiated with UV light. $B($ th caffeine and benzoate absorb at $254 \mathrm{~nm}$. Where these molecules reside on the plate, the amount of UV light 


\section{3}

Fig. 1. Semi-quantitative determination of saccharin, caffeine and sodium benzoate in diet beverage by fluorometric detection. The right four standard samples $(1-4)$ contain $4 \mu g, 2 \mu g, 0.8 \mu g$, and $0.4 \mu \mathrm{g}$ each of saccharin (S), caffeine (C), and sodium benzoate (B) showing up as separate spots from top to bottom. The very left sample (S) is a soft drink sample. The area imaged is $5 \mathrm{~cm} x$ $10 \mathrm{~cm}$. Excitation is at $312 \mathrm{~nm}$ 


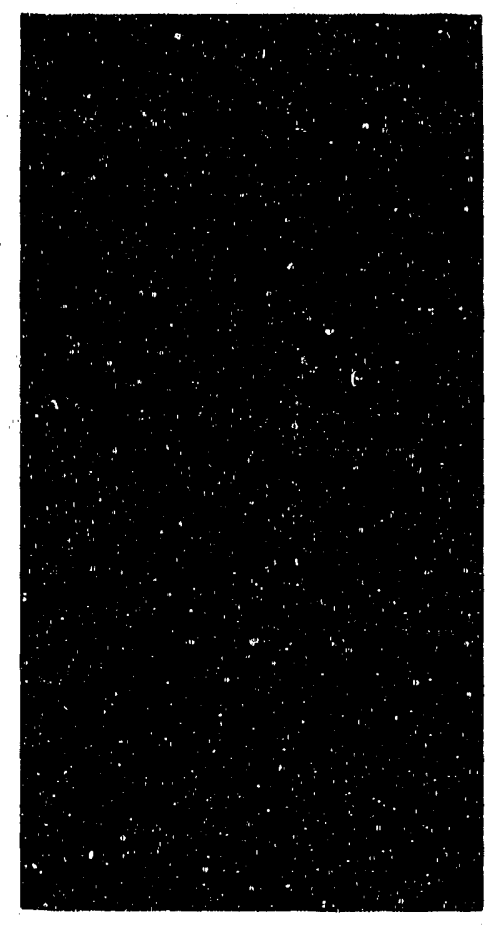


reaching the fluorescent reagent (Nile Blue) is decreased. The fluorescence intensity therefore becomes lower, and "dark" spots are observed. How dark the spots are depends on the concentrations and the molar absorptivities of the components. Caffeine has a higher molar absorptivity than benzoate at $254 \mathrm{~nm}$, so that the caffeine spots appear darker at the same concentration. Commercial TLC plates with a built-in fluorescent reagent are available. These can be used to visualize UV absorbing analytes in the same fashion as described here, i.e., as dark spots. This technique is sometimes called "fluorescence quenching". However, it is in fact an absorption technique and is unrelated to real fluorescence quenching, which alters the quantum yield of the fluorescing species. Commercial plates, however, are not suitable for the other parts of the experiment.

3. Detection by indirect fluorescence $[10-12]$ is illustrated by the spots corresponding to caffeine and benzoate if $312 \mathrm{~nm}$ light is used. At this wavelength, neither caffeine nor benzoate absorbs significantly. Yet, one can observe dark spots on the TLC plate on top of the fluorescence background. The lack of absorption can be verified by observing the spotted TLC plate before development for each of the two components. The 
dark spots form only if the plate is developed. This detection mode is interesting because it in principle allows the visualization of any analyte, whether it absorbs light or noc.

Indirect fluorescence detection is based on competition between the fluorescent reagent and the analyte as they adsorb (partition) on the stationary phase. originally (after pretreatment), there is a uniform distribution of the fluorescent reagent on the TLC plate, giving rise to a uniform fluorescence background. When the analyte moves to a certain part of the plate by development, the partition of the fluorescent reagent between the mobile phase and the stationary phase is altered. Here, the analyte displaces the fluorescent reagent from the stationary phase, producing a region of lower concentration and therefore a dark spot. The amount of fluorescent reagent displaced (i.e., the intensity of the spot) is proportional to the amount of analyte present, so that once again quantitation is possible.

A subtle feature here (for the more sophisticated student) is that indirect fluorescence by displacement also occurs at $254 \mathrm{~nm}$, so a combined effect is observed there. It can be shown that a developed spot (same area) is darker than an undeveloped spot at this 
wavelength. In fact, it takes about eight times the amount of benzoate or three times the amount of caffeine to produce an undev. loped spot that shows the same intensity as the ones in Figure 1. This clearly distinguishes the two visualization mechanisms.

4. Because the plate can be dried and visualized at any time, students can confirm that the $R_{f}$ values for the components are constant. Also, the fact that the spots increase in area when developed further shows the phenomenon of band broadening associated with chromatographic elution.

References

1. Bastian, D. W.; Miller, R. L.; Halline, A. G.; Senftleber, F. C.; Veening, H. I. Chem. Ed. 1977, 54, 766 .

2. Byrd, J. E. J. Chem. Ed. 1979, 56, 809 .

3. McKone, H. T. I. Chem. Ed. 1979, 56, 698 .

4. Lochmuller, C. H.; Wilder, D. R.; Gutknecht, W. F. I. Chem. Ed. $1980,57,381$.

5. Cantwell, F. F.; Brown, D. W. I. Chem. Educ. 1981 , $\underline{58}, 820$.

6. Bohman, O. ; Engdahl, K.-A. ; Johnsson, H. I. Chem. Ed. $1982, \underline{59}, 251$. 
7. Grayeski, M. L.; Woolf, E. J.; Straub, T. S. LC $1985, \underline{3}, 538$.

8. Delaney, M. F.; Pasko, K. M. ; Mauro, D. M.; Gsell,

D. S. ; Korologos, P. C.; Morawski, J.; Krolikowski, L. J.; Warren, F. V. I. Chem. Ed. 1985, 62, 618 .

9. Conklin, A. R. I. Chem. Ed. 1987, 64, 1065.

10. Ma, Y.; Yeung, E. S. Anal. Chem. 1988, 60, 722 .

11. Ma, Y.; Yeung, E. S. I. Chromatogr. 1988, 455, 382.

12. Ma, Y.; Yeung, E. S. Mikrochim. Acta 1988, III; 327. 
SECTION V

\author{
LASER-BASED INDIRECT FLUOROMETRIC DETECTION \\ AND QUANTIFICATION IN \\ THIN-LAYER CHROMATOGRAPHY
}

Introduction

Since thin-layer chromatography (TLC) was described by Kirchner and his group (1), many improvements in all aspects of operation have been made, including high quality, high performance, and multimodal TLC plates, accurate and precise spotting techniques, instrumentalized. development devices, and sophisticated detectors (2-6). These improvements have transformed TLC into a modern separation technique.

Detection and quantification on TLC plates are important considerations. The simplest detection method is based on the human eye aided by a vast array of selective spray reagents, or by the use of plates impregnated with fluorescent indicators to allow components to be detected by absorption at the excitation or emission wavelength. For quantitative analysis, traditionally the plates were scanned by mechanically driven densitometers (7). This is usually a slow procedure. Recently, various methods have 
been suggested for improving the detection limit, speed, and scope of application. These include photoacoustic spectrometry $(8-10)$, flame ionization (11), photothermal deflection (12-13), laser fluorometry (14-15), mass spectrometry (16-19), and computer aided ideo-densitometry $(5,20-21)$. All these detection methods respond to a specific property of the analyte. If the analyte does nut possess that specific property, difficulties in detection will arise. There is thus a reed for a sensitive universal detection scheme for TLC.

Recently, quite a few indirect detection methods for liquid chromatography (LC) have been demonstrated $(22-26)$. The detection mechanisms have been clearly identified (2728). Briefly, the detector responds to some physical property of chromatographic eluent. A constant background signal is then maintained when the analytes are absent. When the analytes elute, displacement of the eluent causes a change in the background signal. So, analytes can be detected indirectly. Following the same principles, the indirect fluorometric detection of anions (29), cations (30), and non-electrolytes (31) in TLC has also been achieved. However, the results so far have not been optimized. The visual observation of the TLC plates (which are illuminated by a UV lamp) is obviously not sensitive compared to instrumental aetection methods. Also, visuai 
obsarvation cannot be reliably used for quantitative measurements. In this section, a laser-based indirect fluorometric detection scheme for TLC is described, which has high sensitivity (picograms) and wide linear dynamic range (more than 2 orders). Reliable scanning is easily performed because the TLC plates and detector are fixed while the excitation laser beam is moved. In this system, a microcomputer controls the $X-Y$ scanning and at some time collects the data. This allows a total data acquisition time of 35 seconds for a data array of $256 \times 64$. There is also an improvement in the signal-to-noise ratio (S/N) resulting from data averaging.

\section{Experimental section}

\section{Apparatus}

The experimental setup for two-dimensional TIC scanning in laser-based indirect fliorometric detection is shown in Figure 1. A He-Ne laser (Uniphase, Manteca, CA) was used as an excitation light source at $633 \mathrm{~nm}$ at a power of $8 \mathrm{~mW}$. In order to maintain a constant laser power, a laser power stabilizer (Cambridge Research and Instrumentation, Cambridge, MA, LS 100) was used. An acousto-optic modulator (Andersen Laboratories, Inc., Bloomfield, CT), which was controlled by a radio frequency 


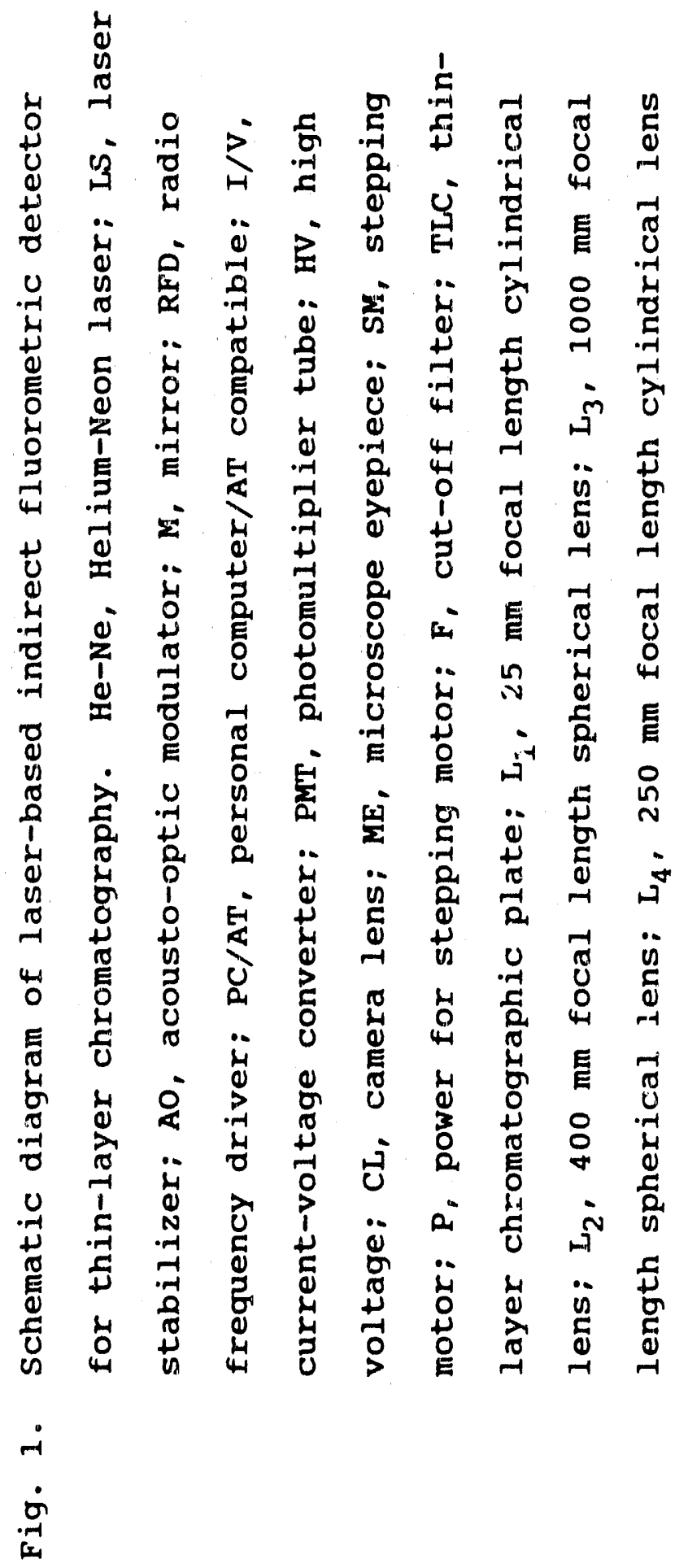




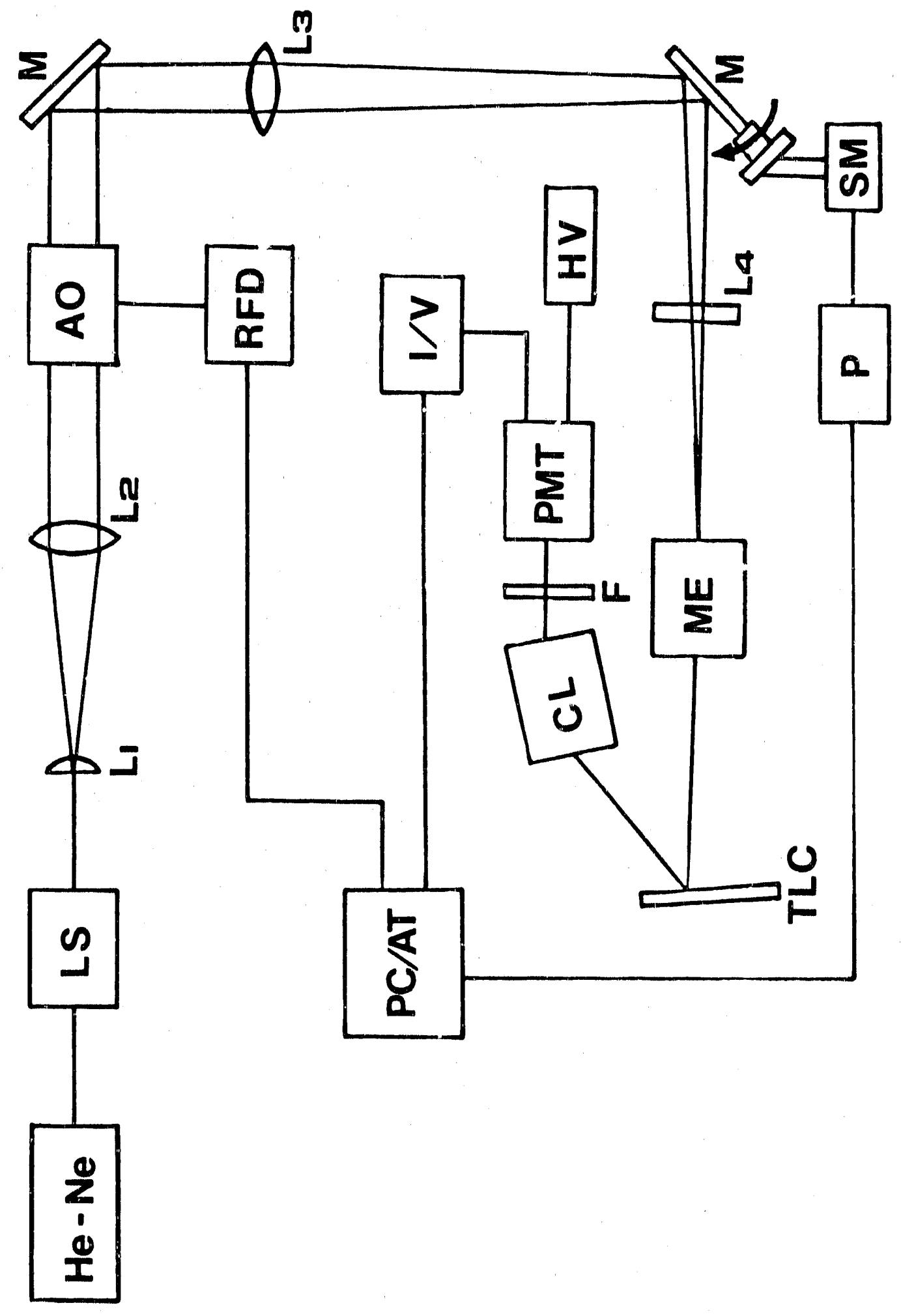


(If) driver, was used to deflect the laser beam. The change in the frequency of $r f$ input to the acousto-optic device causes deflection of the first-order laser beam (32, 33).

The acousto-optic modulator and a rotating mirror combine to scan the laser beam in the horizontal and vertical directions, respectively. To obtain the optimum spatial resolution, we introduced a cylindrical beam expander with $\mathrm{I}_{1}$ and $\mathrm{I}_{2}$ and used long focal length lenses $\mathrm{I}_{3}$ and $\mathrm{I}_{4}$. The focused image onto a microscope eyepiece (12X magnification) was enlarged to an area of $40 \times 50 \mathrm{~mm}$ (horizontal $x$ vertical) with a laser spot size of about 1.5 $\mathrm{mm}$ on the TLC plate.

The fluorescence signal was collected by a camera lens (28-105 mm, f2.8-f3.8, Vivitar Corporation, Santa Monica, $C A$ ), passed through a cut-off filter (to remove scattered $633 \mathrm{~nm}$ light), and directed into a R928 photomultiplier tube (PMT) (Hamamatsu, Middlesex, NJ) operated at $850 \mathrm{~V}$. The output of the PMT is converted into voltage via a resistor and was fed into a data acquisition system consisting of an analog to digital I/O interface (Data Translation, Marlborough, MA, DT 2827) and a microcomputer (IBM, Boca Raton, FL, PC/AT). This system was also used to control the rf output and the stepping motor in order to synchronously scan the laser beam. After each horizontal 
measurement, the laser beam was moved vertically to the next horizontal line by the stepping motor. For our experiment, a data array of 256 x 64 points (horizontal $x$ vertical) was acquired from the entire scan area. Each point was averaged 256 times. Data acquisition takes 35 seconds. Six additional minutes were needed for data averaging and reduction. The final data array was $64 \times 64$ points. The data were tinen normalized to minimize the fluctuations in the background signal.

\section{Chromatography}

A $\mathrm{K}_{6}$ silica gel plate (Whatman, Clifton, NJ) was pretreated with $2 \times 10^{-6}$ M Nile Blue A perchlorate in methanol for 20 minutes. The plate was then aried with a heat-gun. A 0.1 to $1.0 \mu \mathrm{L}$ methanol solution of test sample containing crocein orange $G$ and orange $G$ (Aldrich, Milwaukee, WI) was spotted by a microsyringe (Hamilton, Reno, NV). The thin-layer plate was developed to a distance of about $40 \mathrm{~mm}$ from the origin in a developing solution containing 2-butanol/acetone/water (75:15:10, $V / V)$. After drying, the TLC plate was placed in the apparatus for measurements. For comparison with the detection limit obtained visually, the TLC plate is put under a sodium lamp. 


\section{Results and Discussion}

In indirect fluorometric detection, the limit of detection can be estimated from the following equation (24, 25):

$$
C_{\text {lim }}=C_{F} / D R \cdot T R
$$

where $C_{l i m}$ is the molar concentration of analytes at the detection limit, $C_{F}$ is the molar concentration of the fluorophore, DR is the dynamic reserve (which is defined as the ratio of the background signal to the noise level) and TR is the transfer ratio (which is defined as the number of fluorescing molecules transferred by one analyte molecule). From equation ( 1 ), it is easy to see that if one wants to decrease the detection limit, a more stable background signal (large $D R$ ) and a lower concentration of the fluorophore (smaller $C_{F}$ ) are needed. Even though a large transfer ratio can improve the detection limit, it is generally fixed by the stationary phase, the developing solvent system and the analyte. In our previous papers $(29,31)$, even though we demonstrated indirect fluorometric detection in TLC, the detection limit was not optimum. The human eye has a low contrast, which lowers the DR. In this work, we can easily stabilize the background signal (increase DR) and use lower concentrations of the fluorophor in the pretreating solution (smaller $c_{F}$ ) due to 
the high sensitivity of phototubes. These conditions help to improve the detection limit.

In this work, we chose Nile Blue as the fluorophore because it can be excited by a He-Ne laser. This will greatly enhance cost and convenience considerations in an eventual instrument. Since detection is by indirect fluorescence, the chojce of the fluorophore is quite arbitrary. The analytes were chosen so that they do not absorb at the He-Ne laser wavelength or the fluorescence wavelength, to emphasize the indirect detection mode. Before the TLC plate is developed, the analyte spots do not show any signal. This confirms that displacement of Nile Blue on the plate is responsible for the indirect fluorometric response. The colored analytes also allow us to compare indirect fluorescence with conventional visualization of the spots on TLC plates.

Figure 2 shows a two-dimensional chromatogram of the indirect fluorometric detection of orange $\mathrm{G}$ and crocein orange G. The response is dependent on the analyte because $T R$ is a function of retention factor $\left(R_{f}\right)(25)$. We can see from the figure that $80 \mathrm{pg}$ of each can be detected. However, the conditions are not perfect for quantification because of the fluctuating background. The systematic fluctuations in the background flunrescence signal are caused by variations in the intensity of the laser spot 
98

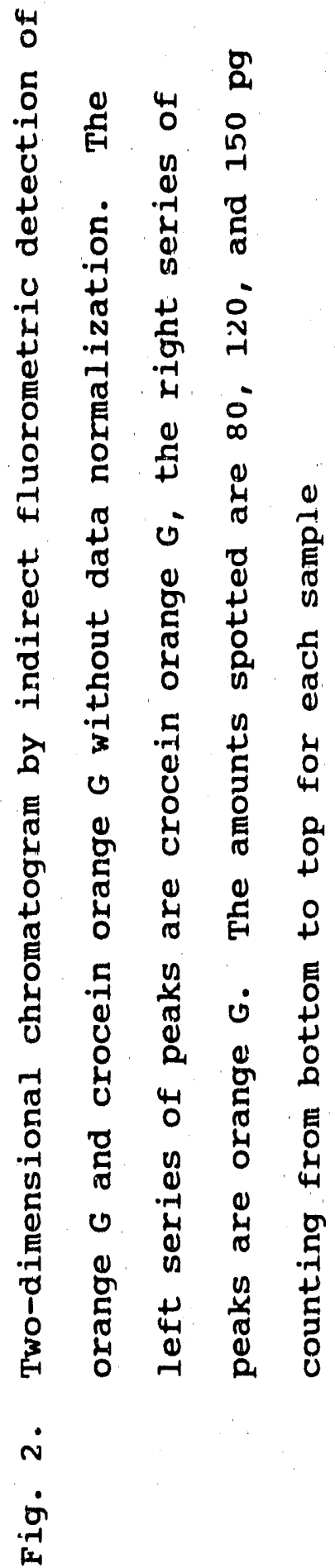


(wo) HICM

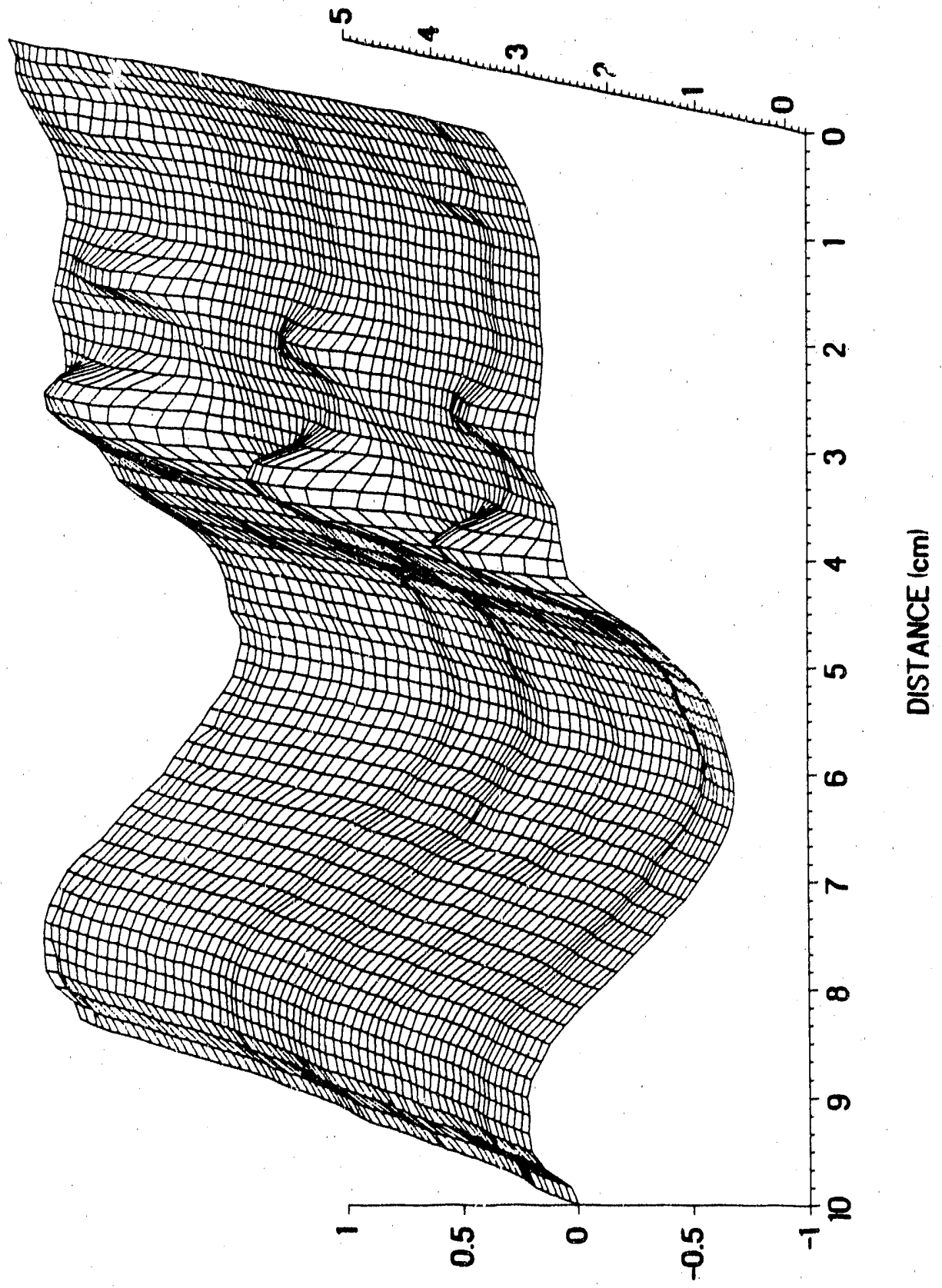

ILISNGINI 
with the angle of deflection and inconsistencies in fluorophore concentration due to the development. To compensate for these fluctuations, the signal can be normalized based on the background levels in a lane in between the sample lanes. The normalized data are shown in Figure 3. The data in Figure 2 and Figure 3 have been inverted to facilitate viewing. Thus, "zero" corresponds to background fluorescence, and "one" would correspond to no fluorescence. Figure 3 shows that signal-to-noise is readily improved by normalization. The detection limit of crocein orange $\mathrm{G}$ is approximately $6 \mathrm{pg}$. This detection limit is over 100 times lower than when the eye is used to view the same dark spots under a sodium lamp (visual indirect flucrometry). This is also 1000 times lower than those reported earlier (31) by using a near-UV fluorophore, about 1000 times better than using the eye to look at the colored spots in room light (direct densitometry) without Nile Blue pretreatment, and 100 times better than photoacoustic detection of the same two analytes $(8,9)$. It should be noted that the detection limit depends on the square root of the number of data points averaged. It is thus possible to reduce the total scan time to 1 second and still achieve detectability in the $40 \mathrm{pg}$ level.

Because of the optical medium, a nonlinear response is usually observed for direct densitometry measurements (34, 
101

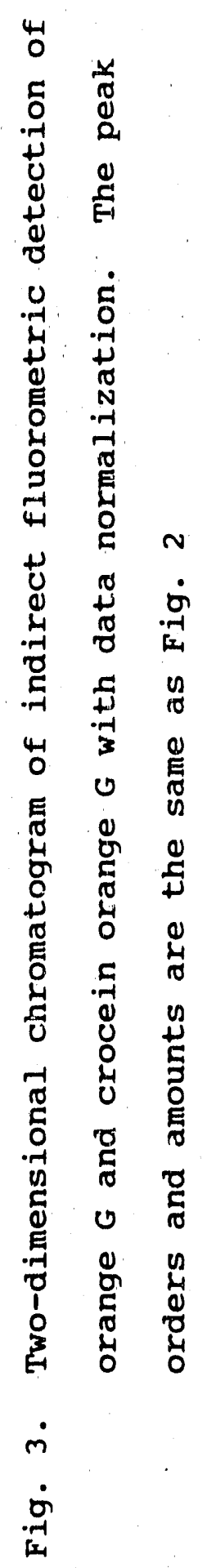


(wo) HLQM

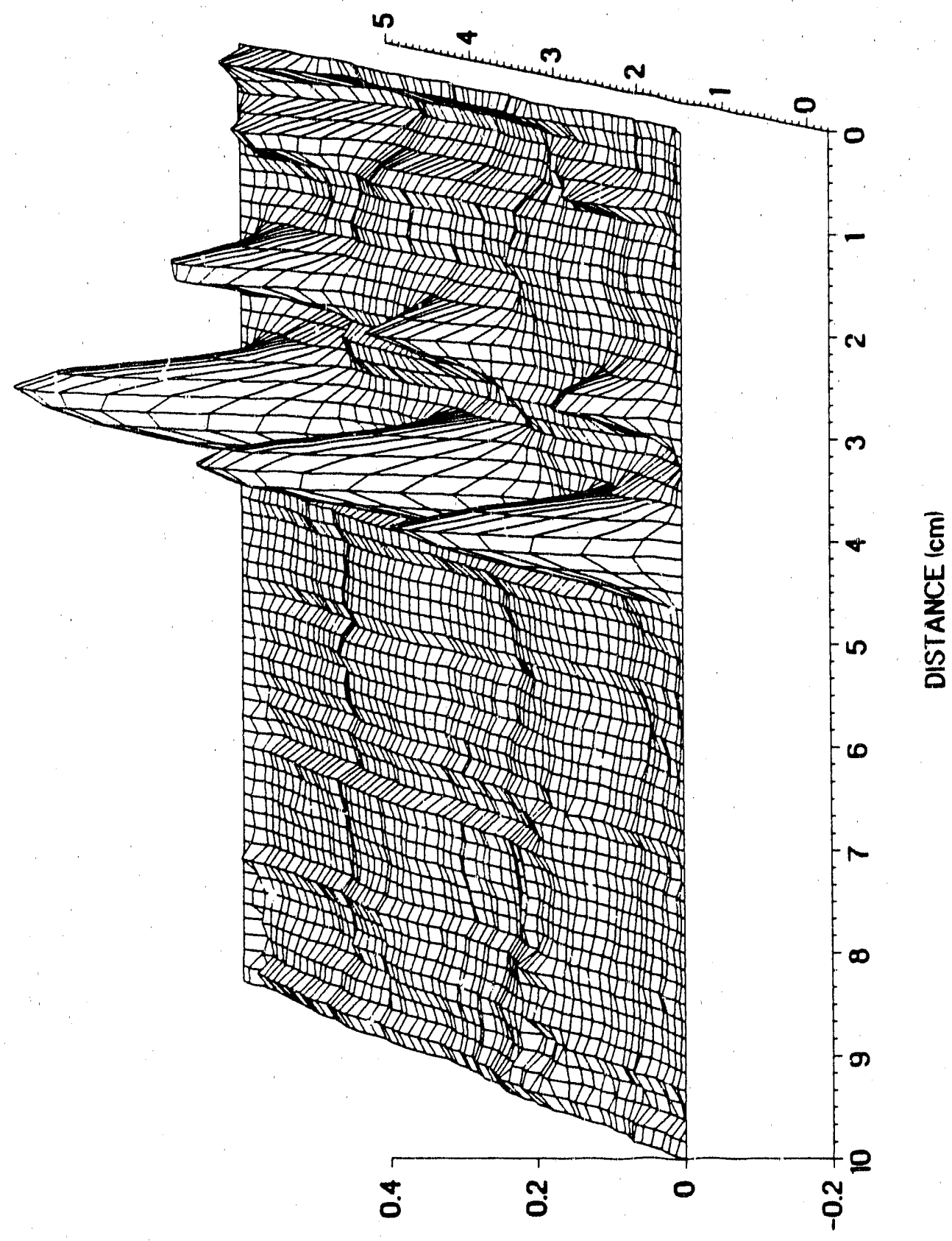

\section{ILISNJINI}


35). This can be corrected by applying the Kubelka-Munk functicn. If the detection mode is based on fluorescence or absorbance, for example, higher concentrations of analytes will zead to nonlinear calibration plots due to self-absorption, self-quenching, and detector saturation. In indirect fluorometric detection, the response is not based on the analyte itself. A linear response is therefore easier to obtain. Figure 4 shows a calibration curve for crocein orange $G$. A line with unit slope is shown for comparison. Bused on the linear regression criteria described by Johnson (36), linearity was observed over two orders of magnitude with relative deviations of approximately 10\%. Note that the calibration curve was based on the peak volume of the TIC spots in Figure 3. This properly accounts for band broadening due to development. The peak volume can be estimated by determining the peak area under a single scan through the peak raised to the $3 / 2$ power. Due mostly to variations in the concentrations of the fluorophore from plate to plate, and partly due to variations in light collection geometry, it was neressary to include a standard spot on each TLC plate to allow normalization among different plates. If the concentration of the analyte is too high (beyond 3.2 in Figure 4), the calibration plot begins to show nonlinearity. This happens because the TR in equation (1) 
104

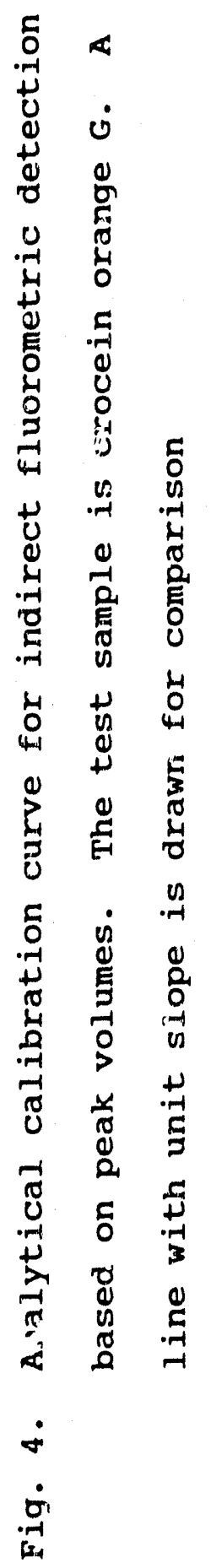


105

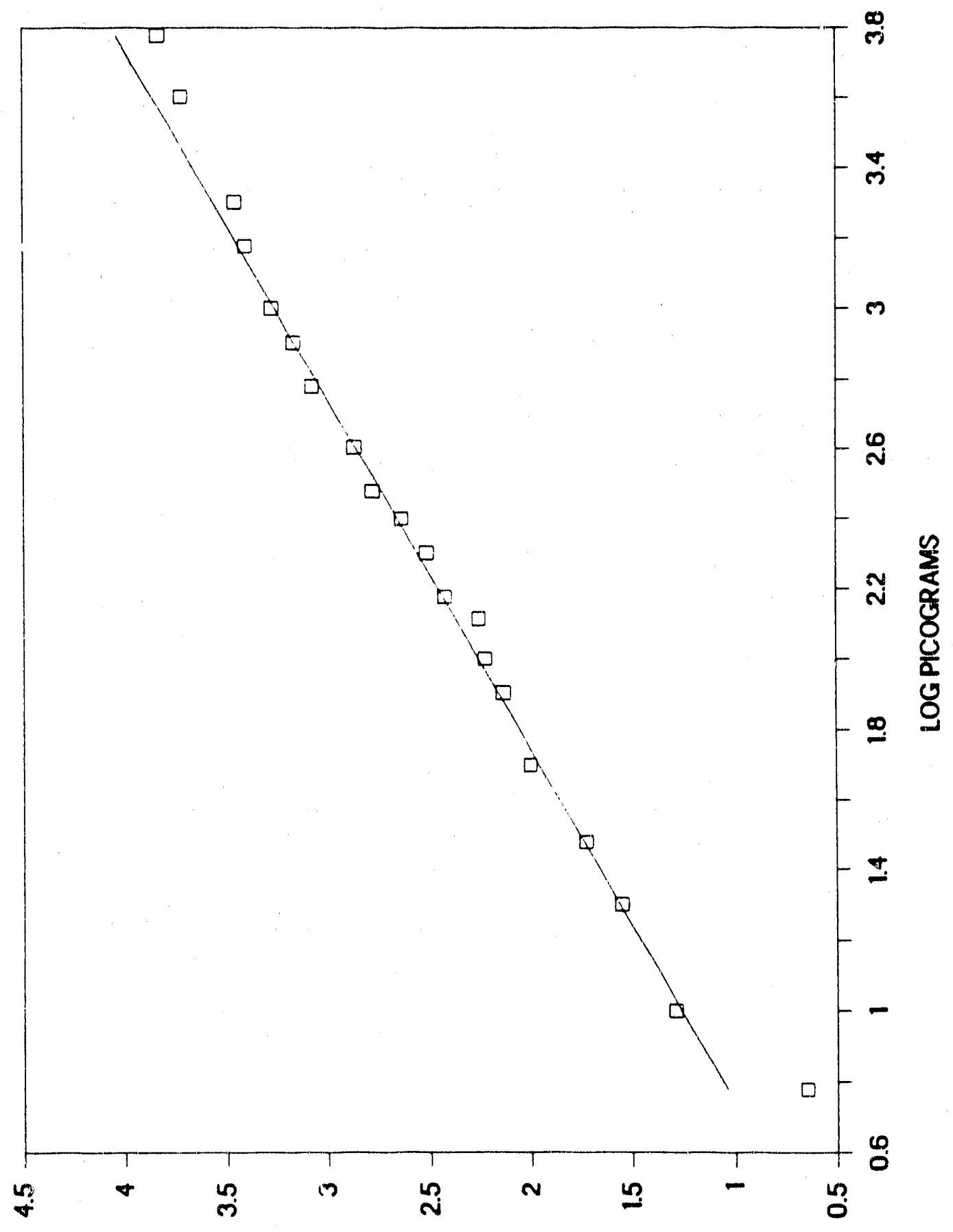

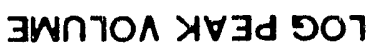


will change at higher concentrations. The signal also falls off at the low concentration end, probably because of difficulties in integration and in background normalization for small signals. However, the linear range achieved is large enough for this approach to be broadly applicable.

\section{Conclusion}

We have developed a laser-based indirect fluorometric detection scheme for TLC. The method is universal, very sensitive, and has a large linear dynamic range. The laser and the optics used should allow the construction of a rugged, fully automated instrument eventually. Presently, commercial TLC plates have to be pretreated with the fluorophor to allow indirect detection. It is conceivable that this step can be incorporated into the manufacturing process. The entire process of TLC separations is then substantially simplified, i.e., one can avoid the tedious and unreliable derivatization steps completely.

Registry No.: Nile Blue A perchlorate, 53340-16-2; Crocein orange G 1934-20-9; Orange G, 1936-15-8. 
References

1. Kirchner, J. G.; Miller, J. M.; Keller, G. J. Anal. Chem. 1951, 23, 420 .

2. Donovan, J.; Gould, M.; Majors, R. E. LC-GC 1987, $\underline{5}$, 1024.

3. Jaenchen, D. E.; Issaq, H. J. I. Lig. Chromatogr. $1988,11,1941$.

4. Jaenchen, D. E. Am. Lab. $1988, \underline{3}, 66$.

5. Dallas, F. A. A.; Read, H.; Ruane, R. J.; Wilson, I. D. Recent Advances in Thin-Layer Chromatography; Plenum: New York, 1988.

6. Touchstone, J. C. I. Chromatogr. SCi. 1988, 26, 645.

7. Touchstone, J. C.; Sherma, J. Densitometry in ThinLayer Chromatography: Practice and Applications; Wiley: New York, 1979.

8. Kawazumi, H. ; Yeung, E. S. Appl. Spectrosc. 1988, 42, 1228.

9. Kawazumi, H.; Yeung, E. S. Appl. Spectrosc. 1989, 43, 249 .

10. Imaeda, K.; Ohsawa, K.; Uchiyama, K.; Nakamura, S. ; Tokieda, T. Anal. Sci. 1986, 2, 9.

11. Ranny, M. Thin-Layer Chromatography with Flame Ionization Detection; Reidel: Boston, 1987.

12. Chen, I. I.; Morris, M. D. Anal. Chem. 1984, 56, 19. 
13. Chen, I. I.; Morris, M. D. Anal. Chem. 1984, 56, 1674 .

14. Huff, P. B.; Sepaniak, M. J. Anal. Chem. 1983, 55, 1992 .

15. Belenkii, B. G.: Gankina, E. S. J. Chromatogr. 1986, $\underline{365}, 315$.

16. Chang, T. T.; Andrawes, F.; Kaiser, R. E. Proceedings of the Third International symposium on Instrumental HPTLC; Institute for Chromatography: Bad Burkheim, West Germany, 1985; pp. 427-433.

17. Chang, T. T.; Lay, J. O.; Francel, R. J. Anal. Chem. $1984, \underline{56}, 109$.

18. Ramaley, L.; Vaughn, M.-A.; Jamieson, W. D. Anal.

Chem. 1985, 57., 353 .

19. Kraft, R.; Otto, A.; Zoepfl, H. J.; Etzold, Gerhard Biomed. Environ. Mass Spectrom. 1987, 14, 1.

2C. Ford-Holevinski, T. S.; Radin, N. S. Anal. Biochem. $1985, \underline{150}, 359$.

21. BE lchamber, R. M. ; Read, H. ; Roberts, J. D. M. I. Chromatogr. 1987, 395, 47.

22. Bobbitt, D. R.; Yeung, E. S. Anal. Chem. 1984, 56, 1577.

23. Mho, S. I.; Yeung, E. S. Anal. Chem. 1985, 57, 2253.

24. Takeuchi, T.; Yeung, E. S. I. Chromatogr. 1986, 370, 83. 
25. Takeuchi, T.; Yeung, E. S. I. Chromatogr. 1986, 366, 145.

26. Pfeffer, W.: Takeuchi, T.; Yeung, E. S. Chromatographia, $1987,24,123$.

27. Ishii, D.; Takeuchi, T. I. Liq. Chromatogr. 1988, 11, 1865.

28. Yeung, E. S. Accounts Chem. Res. 1989, 22, 125.

29. Ma, Y.; Yeung, E. S. Anal. Chem. 1988, 60, 722.

30. Ma, Y.; Yeung, E. S. Mikrochim. Acta 1988 III, 327.

31. Ma, Y.; Yeung, E. S. I. Chromatogr. 1988, 455, 382.

32. Lekavich, J. Lasers and Applications 1986, 4, 59.

33. Young, M. Optics and Lasers; springer-Verlag: Berlin, $1986 ;$ p. 193.

34. Ebel, S.; Glaser, E. I. High Resolut. Chromatogr. Chromatogr. Commun. $1979,2,36$.

35. Costanzo, S. J.; Cardone, M. J. J. Liq. Chromatogr. 1984, I. 2711.

36. Johnson, D. C. Anal. Chim. Acta 1988, 204, 1 . 


\title{
SECTION VI
}

\begin{abstract}
THE EFFECT OF ULTRASOUND ON THE SEPARATION OF DNA FRAGMENTS IN AGAROSE GEL ELECTROPHORESIS
\end{abstract}

Introduction

since its first use in 1966 (1), interest in and the applications of electrophoresis of DNA fragments in agarose gel have grown rapidly (2). Nowadays, agarose gel electrophoresis has become a standard technique with high resolving power for the analysis of DNA structure, for. example for the determination of the length of DNA fragments obtained by the action of restriction enzymes. The electrophoretic mobility (1) of DNA fragments is influenced by various parameters-molecular weight (3-5), gel concentration $(6,7)$, temperature $(7,8)$, electric field $(6,7)$, and DNA-agarose affinity $(9)$. A comprehensive study of the influence of these main parameters has been reported (10). In this section, a new effect on the electrophoretic mobility of DNA fragments in agarose gels is investigated, viz. the influence of ultrasound. Ultrasound techniques have long been used in medicine and biology $(11,12)$, including medical diagnosis, surgery, dental applications, scission of DNA, homogenizatior., celz 
disruption, and so on. In recent years, the applications of ultrasonics in chemistry have also mushroomed. A large num'ser of articles have been published which describe a variety of applications of ultrasound in chemical processes (13-19). These include synthesis (organic, organometallic and inorganic), polymer chemistry (degradation, initiation and copolymerization) and some aspects of catalysis. A detailed description on these areas has appeared in a recent book (20). Up to now, ultrasonics has not found extensive uses in analytical chemistry, even though some researchers (21) have used ultrasonics to identify liquids, analyze mixtures of liquids, determine the concentration of solution and dispersion, identify and analyze gases by determining the velocity of ultrasound.

In this work, ultrasound energy is directed to excite a tube gel while electrophoresis is proceeding. We found that the ultrasound improves the separation of DNA ragments without degrading them, a phenomenon which is totally different from that of ultrasonic scission (22).

Experimental section

\section{Materials}

High range DNA fragments (hDNA) standard and ultra pure DNA grade agarose were obtained from BIO-RAD 
laboratories (Richmond, CA). Ethidium bromide was purchased from Kodak (Rochester, NY). Phosphoric acid (85\%) and disodium ethylenediamine tetraacetic acid (EDTA) were obtained from Fisher Scientific (Fair Lawn, NJ). Glycerol and bromophenol blue were purchased from sigma (St. Louis, MO).

\section{Equipment set-up}

A schematic diagram of the experimental arrangement used is shown in Figure 1. A Branson sonifier 250 (Branson Ultrasonics, Danbury, CT) was used to produce ultrasound (20 kHz, 200W output maximum). A 1/8" micro ultrasonic tip was used in our experiment.

\section{Gel tubing treatment}

Glass tubes $(10 \times 0.1 \mathrm{~cm} \mathrm{i.d.} \times 0.2 \mathrm{~cm} 0 . \mathrm{d}$.$) were used$ for electrophoresis. Before using, we treated the internal surface of the tube with $1 \%$ PS-255 polymer. The specific procedure is the following: glass tubes were cut to $12 \mathrm{~cm}$ long: 0.1 gram of PS-255 (dimethyl (0.5-1\%) methylvinylsiloxane copolymer, gum) (Petrarch systems Inc., Bristol, PA) was dissolved in $10 \mathrm{~mL}$ pentane (Eastman Kodak Co., Rochester, NY) with electromagnetic stirring for about an hour; $1 \mathrm{mg}$ of dicumyl peroxide (Pfaltz Bauer Inc., Waterbury, CT) was then dissolved in the solution with 
Fig. 1. Schematic diagram of tube gel electrophoresis of DNA fragments with sonication. A, J, running buffer solutions; $B$, pump (flow rate $=1.5$ $\mathrm{mL} / \mathrm{min}) ; \mathrm{C}, \mathrm{I}$, Pt electrodes; $\mathrm{D}$, microultrasonic tip (1/8"); E, ultrasonic power supply; F, ultrasonic and electrophoresis cell; G, chromelalumel thermocouple; $H$, microvoltmeter to monitor the temperature change 


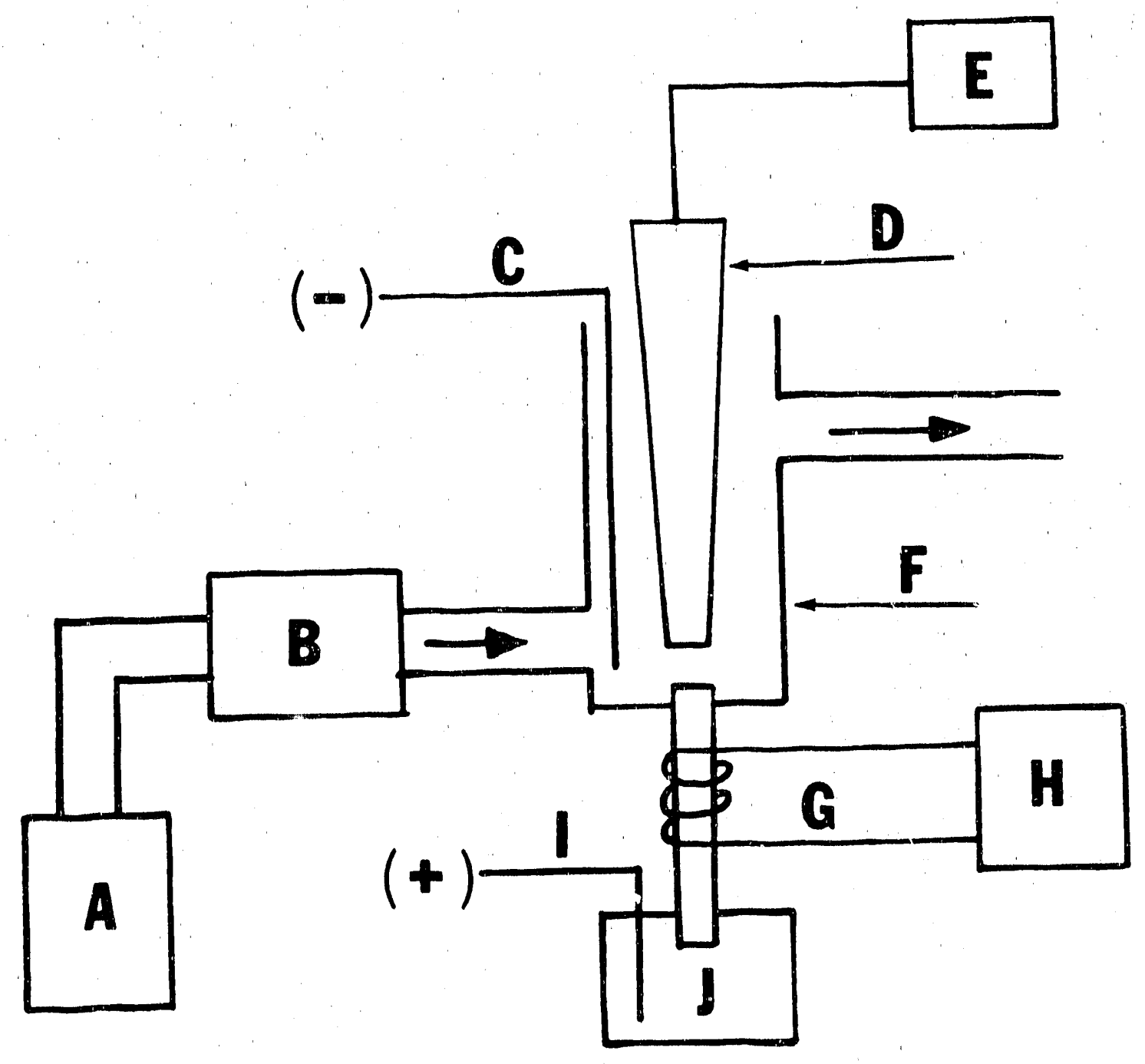


about 10 minutes stirring; the polymer solution was used to fill the tubes which have one of the ends closed; the tubes were put in a gas chromatographic oven for about one hour at $40^{\circ} \mathrm{C}$ until all the solvent was evaporated; the oven temperature was raised to $170^{\circ} \mathrm{C}$ gradually $\left(1.5^{\circ} \mathrm{C} / \mathrm{min}\right)$ and maintained for about $5 \mathrm{~min}$. at this temperature; the tubes were then stored for later use. The purpose of this extensive treatment is two-fold: (1) to prevent the interaction of DNA fragment with the functional group (Si$\mathrm{OH})$ on the glass tubing wall, and (2) to prevent the gel frow sliding out when the ultrasonic tip was in operation.

Preparation of reagents and gel

The electrophoresis running buffer solution was $0.08 \mathrm{M}$ tris-phosphate containing $2 \mathrm{mM}$ EDTA and $0.5 \mu \mathrm{g} / \mathrm{mL}$ ethidium bromide ( $\mathrm{pH} 7.5)$. A detailed preparation procedure was described elsewhere (23). The running buffer solution should be degassed before using. High range DNA standard $(0.4 \mu \mathrm{g} / \mu \mathrm{L})$ were diluted to $1 / 6$ the concentration with the sample loading buffer, which was the same as the running buffer except for the omission of ethidium bromide. The sample was adjusted to $8 \%$ glycerol $-0.025 \%$ bromophenol blue before loading. Agarose tube gels were prepared in a similar way to that of Sharp et al. (24), except that we use a $0,75 \%$ agarose gel and tris-phosphate buffer. After 
gelatinization, $1 \mathrm{~cm}$ of both ends of the tubing was cut off to ensure a flat gel surface, the final tube length is 10 $\mathrm{cm}$. A $1-\mathrm{mm}$ deep sample well was made on the top gel to load the sample.

\section{System operation}

$0.6 \mu \mathrm{L}$ of sample solution was injected into the sample well of the tube gel. One end of the gel (at which the sample was loaded) was connected to the ultrasonic cell, F. The other end was connected to a running buffer pool, $J$, with the tube in a vertical position. A constant voltage $(100 \mathrm{~V}, 2 \mathrm{~W})$ was used throughout the experiment. After the electrophoresis power supply was turned on for 5 min., the ultrasound power supply, E, was turned on. At the same time, the running buffer solution, $A$, was pumped to the cell via $B$ to remove the heat produced by micro ultrasonic tip, D. The operating power of the ultrasonic tip was in the 10W-40W range. Ultrasound was introduced for $40 \mathrm{~min}$. and electrophoresis was carried out for 45 min. at room temperature. A chromel-alumel thermocouple, G, was used to monitor the temperature change throughout the process. A $5 \mathrm{~min}$. delay between the start of electrophoresis and the start of ultrasound is necessary, otherwise the DNA sample may be blown out of the sample we 11 . 
Results and Discussion

Figure 2 demonstrates the effect of ultrasound on the separation of hDNA fragments in gel electrophoresis. The bright bands are due to the fluorescense of ethidium bromide which was intercalated in the DNA fxagments. We can see clearly that the DNA fragments with ultrasound excitation migrate faster than those without ultrasound. one possible reason for this phenomenon is that ultrasound decreases DNA-agarose affinity which causes the DNA fragment bands to be retarded and to broaden. A model for DNA-agarose interaction has been proposed by smith et al. (9):

$$
\mathrm{U}_{\mathrm{R}}=\mathrm{U}_{\mathrm{R}}^{\mathrm{S}}+\mathrm{U}_{\mathrm{O}} \mathrm{K}_{3} /\left(1+\mathrm{K}_{3} \mathrm{~K}_{5} \mathrm{U}_{\mathrm{O}}(\mathrm{L})\right)
$$

where $U_{R}$ is the observed band mobility, $K_{5}$ is the DNA-gel. interaction constant which can be considered a retardation coefficient due to the force of the DNA-gel interaction, $U_{O}$ is the mobility in free solution and $U_{R}{ }_{R}$ is the mobility in the gel due to the sieving action. It is clear from equation (1) that if ultrasound somehow decreases the DNAgel interaction, i.e., $\mathrm{K}_{5}$ is smaller, the bands will migrate faster.

Another possible reason is that ultrasound may 
Fig. 1. Schematic diagram of tube gel electrophoresis of DNA fragments with sonication. A, J, running buffer solutions; B, pump (flow rate $=1.5 \mathrm{~mL} / \mathrm{min}) ; C, I$, Pt electrocies; $D$, microultrasolic tip (1/8"); E, ultrasonic power supply; F, ultrasonic and $\in$ iectrophoresis cell; $G$, chromel-alumel thermocouple; $H$, microvoltmeter to monitor the temperature change 


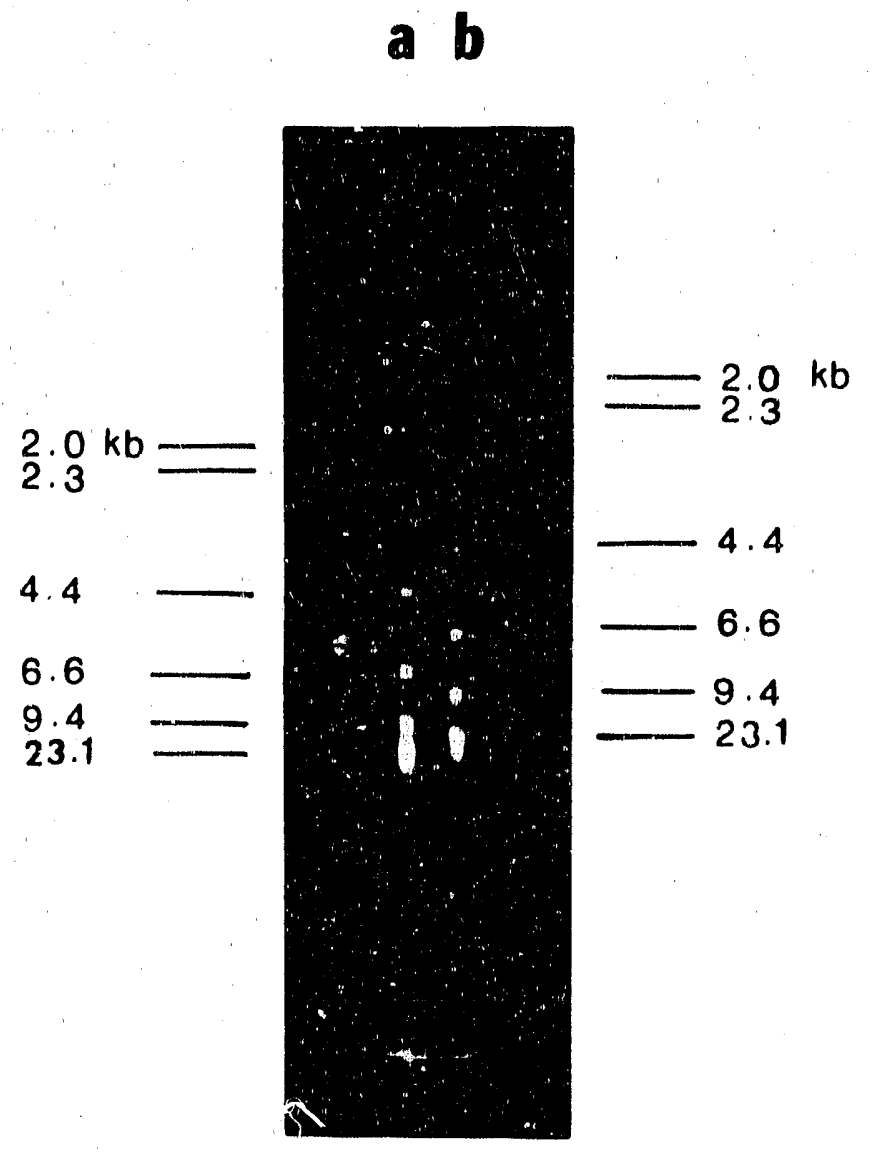


decrease the intrinsic viscosity of DNA fragments and/or the buffer solution. The relationship between DNA mobility and viscosity can be expressed as follows (1.0):

$$
\mu=\frac{Q(T,[C])}{\epsilon(\eta, T, C)} g(L, E)
$$

where $\mu$ is the electrophoretic mobility, $Q$ (T, [C]) is the effective charge of the DNA which is sensitive to temperature (T) and ionic strength of the buffer [C]. $\epsilon(\eta$, $T, C)$ is the frictional force per unit length of DNA which depends on the viscosity $(\eta)$, temperature (T) and gel concentration (C). $g(L, E)$ is a parameter which is a function of the contour length (L) and of the electric field. Equation (2) shows that a decrease of viscosity will cause an increase of DNA mobilily. Some researchers have already demonstrated that the intrinsic viscosity of a sonicated DNA solution decreases rapidly with the increase in ultrasonic radiation energy (22). However, that is because irreversible degradation of the DNA has occurred, as evidenced by the change to a smaller average molecular weight. To confirm that a different phenomenon is responsible for our observations, we performed a control experiment in which the ultrasound was turned on for an identical amount of time (40 min.) but with the electrophoresis current off. After irradiation, electrophoresis was continued for a total of $45 \mathrm{~min}$. as 
before. In that case, the bands migrated exactly as if no ultrasound was ever applied. This proves that in our experimental arrangement the DNA fragments were not degraded. The ultrasound actually affected the mobility of the fragments in the gel during electrophoresis.

A third possibility is that there is a temperature change (increase) as a result of the application of ultrasound energy. This then affects the melting of the DNA and/or change the viscosity as prescribed by equation (2). However, according to the thermocouple in contact with the tube, there was no measurable temperature change at these ultrasound power levels when proper circulation of the buffer was maintained. We note that the highest temperature is at the tip of the soricator. That part is cooled very efficiently by circulation.

Fig. 3 shows the relationship between the percentage increase of DNA migration velocity and ultrasonic power. It is easy to see that the DNA bands migrate further with the increase of uitrasonic power. An increase of about $22 \%$ for $6.6 \mathrm{~kb}$ and $2.0 \mathrm{~kb}$ DNA fragments can be achieved at 40 watts of ultrasonic power. However, we cannot increase the ultrasonic power above $40 \mathrm{~W}$ in this experimental arrangement because bubbles produced by the ultrasonic tip become excessive. They may block the gel or even destroy the gel. 
122

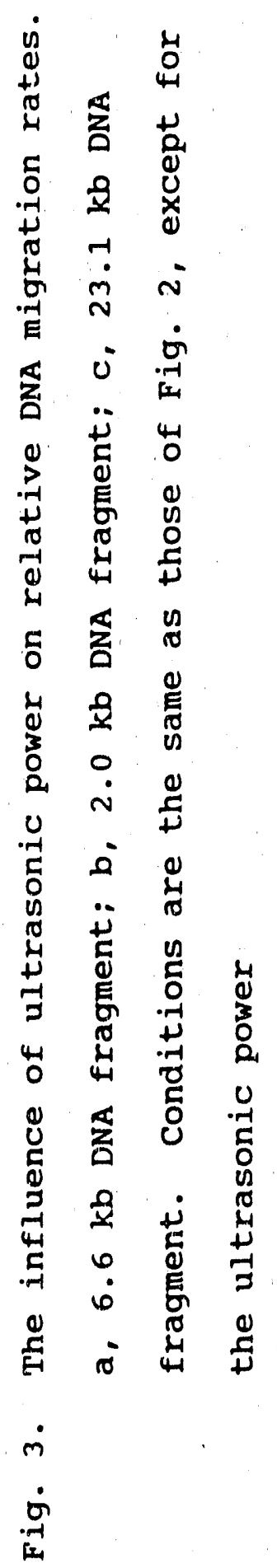




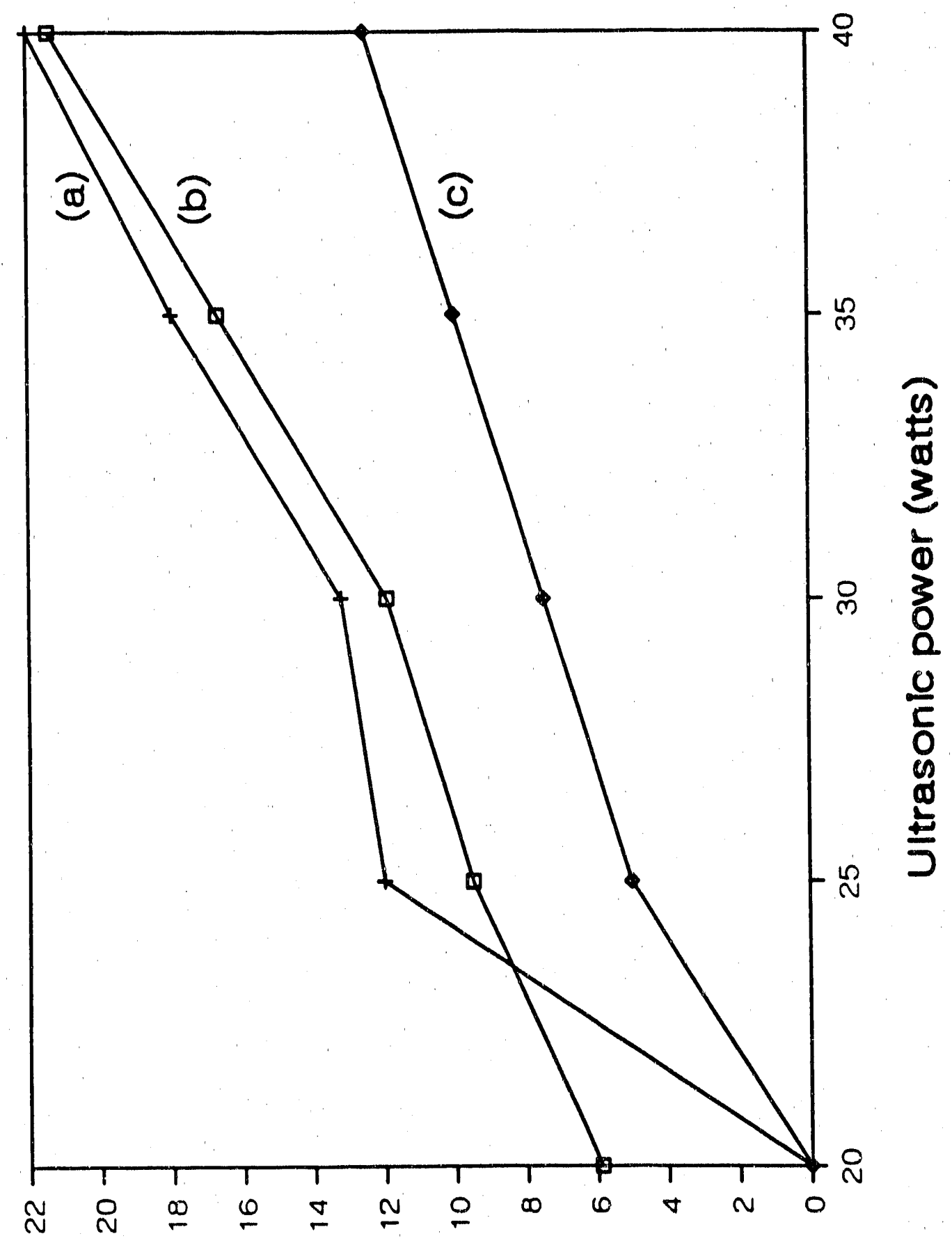

sө|вג UO!f 
Fig. 4 demonstrates the effect of ultrasound on the bandwidth of high range DNA fragments. It is found that the bandwidth of the $23.1 \mathrm{~kb}$ DNA fragment decreases with an increase in ultrasonic power. This is consistent with the explanation above that ultrasound can decrease the DNAagarose gel affinity, reducing the amount of band tailing. The smaller fragments on the other hand showed very little change in bandwidth in these exper nents.

The relationship between the percentage increase in DNA migration rate and the distance of the ultrasonic tip to the gel is shown in Fig, 5. The dependence is not monotonic when the distance of the ultrasonic tip to the gel was increased. This can be explained in two ways. First, the ultrasound energy coming from the microtip might be focused due to the geometry used. Being too close to the gel may in fact result in lower transferred acoustic pover. Second, the ultrasound we used has a wavelength of abcut $5 \mathrm{~cm}$ (speed of ultrasound in buffer solution is about $1000 \mathrm{~m} / \mathrm{s}$ ) so that $2.5 \mathrm{~cm}$ and $5 \mathrm{~cm}$ are standing wave nodes. When the distance of the ultrasonic tip to the gel is near these numbers, the ultrasonic power is actually smaller than at other distances. Naturally, ultrasonic power generally decreases with the increasing distance. This explains the behavior at large distances. Unfortunately, 


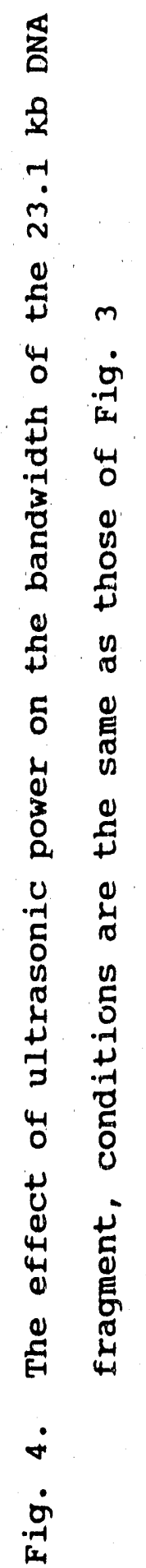




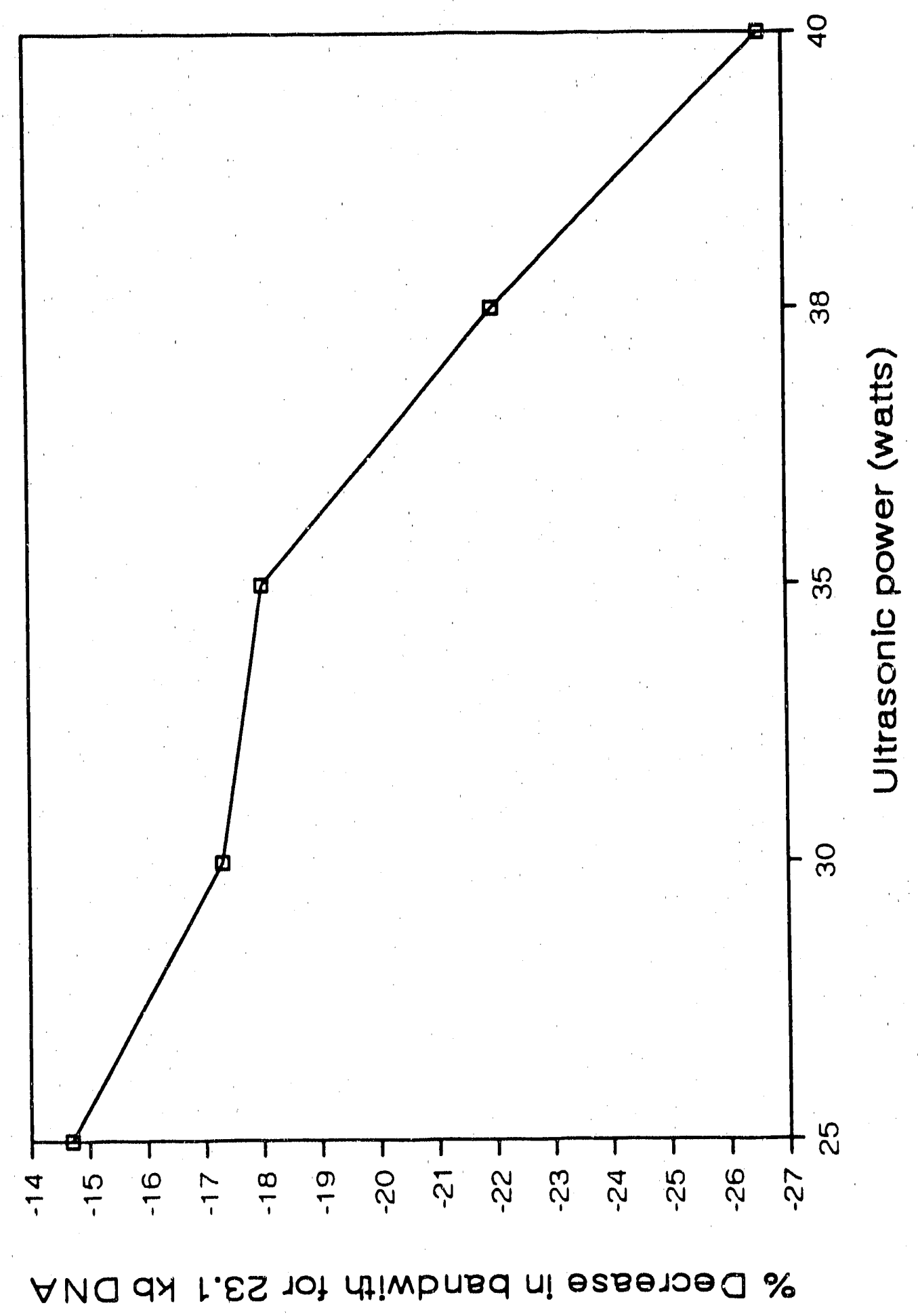




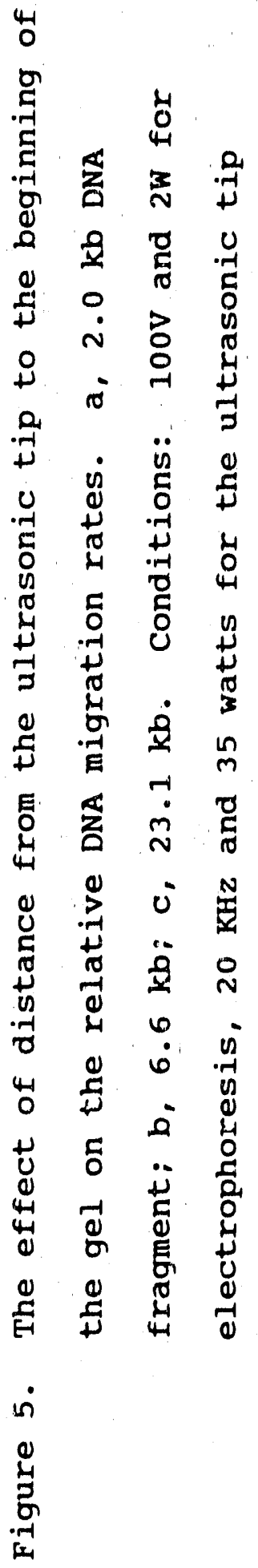




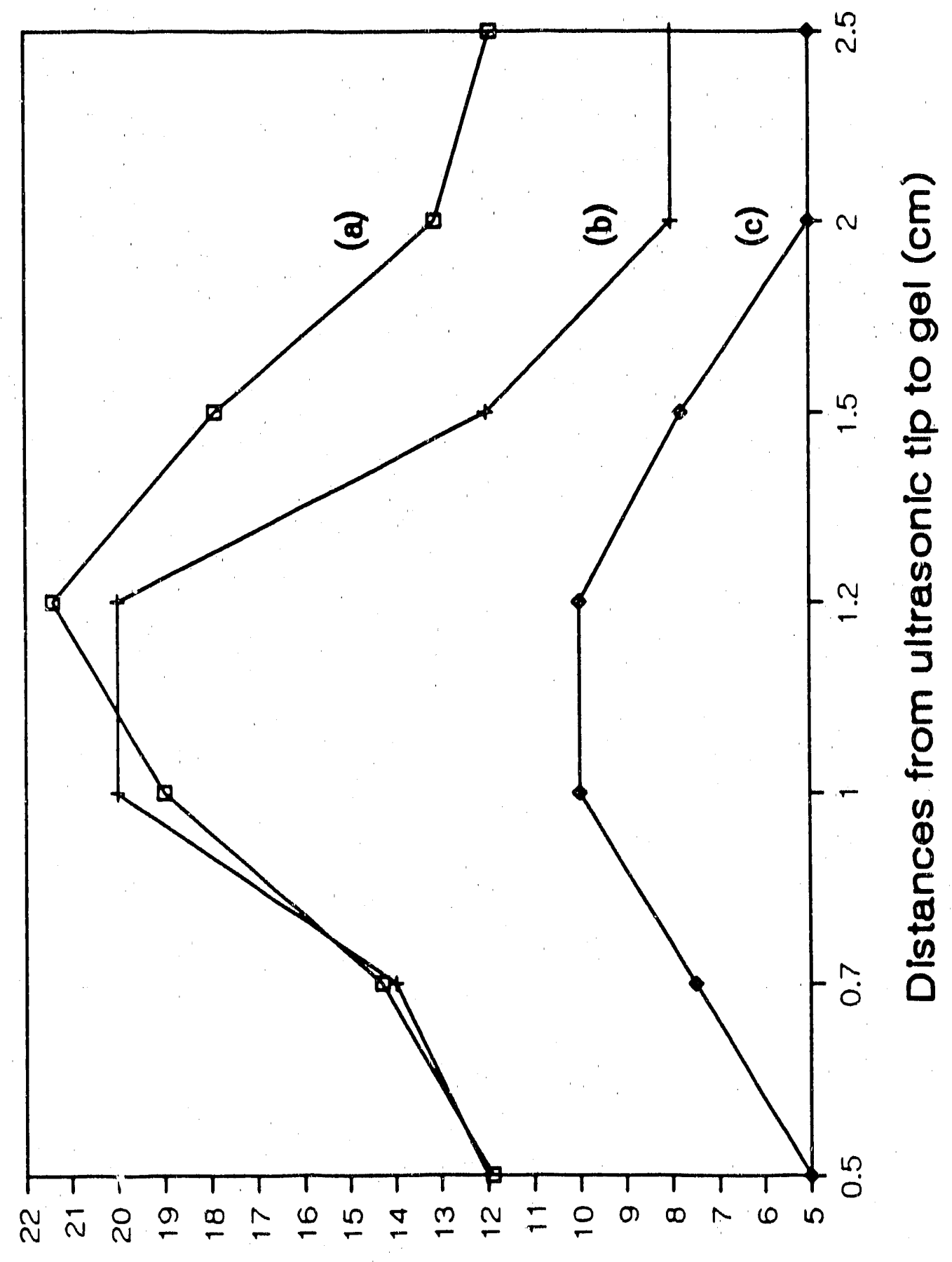

sө!в UO!f 
we were unable to map out the actual distribution of acoustic power in the gel to provide quantitative correlations. Fig. 5 also shows that heating of the gel cannot be the main reason for these changes in migration rates. If so, one would have found a monotonic decrease with increasing separation of the probe, in accordance with the likely temperature gradient away from the sonicator tip.

In conclusion, we have characterized the influence of ultrasound on the migration of DNA fragments in agarose gel electrophoresis. The effect is to increase the migration rate and to sharpen the bands. Both of these are desirable for enhancing the separation power of gel electrophoresis. The phenomenon here is quite different from the one reported for size-exclusion chromatography (25), where retention times are unaffected but peak widths become broader. The magnitude of the effect is limited by the available sonicator used here. It is reasonable to expect that by adjusting the ultrasound frequency, power, and location, one may be able to magnify these effects to introduce unique selectivity in DNA separations. 
References

1. Thorn, H. V. J. Mol. Biol. 1967, 24, 203.

2. Serwer, P. Electrophoresis 1983, $4,375$.

3. Fischer, P.; Dingman, C. W. Biochemistry 1971, 10, 1895.

4. Southern, E. M. Anal. Biochem. 1979, 100, 319.

5. Eämondson, S. P.: Gray, D. M. Biopolymers 1984, 23, 2725 .

6. Stellwagen, N. C. Biochemistry 1983, 22, 6180 .

7. Serwer, P.; Allen, J. L. Biochemistry $1984,23,922$.

8. Woolley, P. Electrophoresis $1987,8,339$.

9. Smith, S. S.; Gilroy, T. E.; Ferrari, F. A. Anal. Biochem. 1983, 128, 138.

10. Hervet, H.; Bean, C P. Biopolymers $1987,26,727$.

11. Brown, B.; Gordon, D. Ultrasonic Techniques in Biology and Medicine; Charles C. Thomas: Springfield, IL, 1967.

12. Suslick, K. S. Ultrasound; VCH: New York, 1988.

13. Boudjouk, P. Nachr. Chem. Iech. Lab. 1983, 31, 78.

14. Mason, T. J. Ultrasonics 1986, 24, 245.

15. Suslick, K. S. Modern Synthetic Methods 1986, 4, 1.

16. Bremner, D. Chem. Br. 1986, 22, 633.

17. R. S. C. Sonochemistry Symposium, University of Warwick, 1986; Ultrasonics 1987, 25, January. 
18. Lorimer, J. P.; Mason, T. J. Chem. Soc. Rev. 1987, 16. 239 .

19. Lindley, J.; Mason, T. J. Chem. Soc. Rev. 1987, 16, 275 .

20. Mason, T. J.; Lorimer, J. P. Sonochemistry; Ellis Horwood: Chichester, England, 1988.

21. Asher, R. C. Ultrasonics $1987,25,17$.

22. Fu'ndome, K.; Yamaoka, K.: Nishikori, K.; Takahashi, T.; Yamamoto, 0. Polymer Journal 1986, 18, 71.

23. Ogden, R. C.; Adams, D. A. Methods in Enzymology $1987, \underline{152}, 78$.

24. Sharp, P. A.; Sugden, B.; Sambrook, J. Biochemistry $1973,12,3055$

25. Synovec, R. E. ; Yeung, E. S. J. Chromatogr. 1987, $\underline{388}, 105$. 
GENERAL SUMMARY

This dissertation is mainly devoted to the development of indirect fluorometric detection technique for thin-layer chromatography (TLC). It has been demonstrated that this technique works viry well for anions, cations, nonelectrolytes and biological samples. With this universal detection method, nanomoles or subnanomoles of samples can be easily detected by human eyes without using any spraying reagents or derivatization process. This detection technique is especially suitable for detecting the nonfluorescing and nonabsorbing samples, which may cause a lot of trouble for other detection techniques. However, human eyes are not the perfect detector. The visual observation of the TLC plates (which are illuminated by a UV lamp) is obviously not sensitive compared to instrumental detection methods, and can not cut off the background noise reflecting from TLC plates to maintain a reasonable signal to noise ratio. Also, visual observation can not be reliably used for quantitative measurements. Therefore, this dissertation develops a laser-based indirect fluorometric detection method. This laser-based detection technique can decrease the detection limit to pg level, which is 100-fold lower than that by using human eyes as a detector for same samples. It is also shown that 
laser-based indirect fluorometric detection technique, two orders of magnitude of linearity can be achieved. The improvement of detection limit by laser-based detection technique is mainly due to the increase of dynamic reserve (which is defined as the ratio of the background signal to noise level), cecrease of fluorophore concentration (smaller $c_{f}$ ) and data averaging by using a computer. In addition, this dissertation also investigated the effect of ultrasound on the separation of DNA fragments in agarose gel electrophoresis. It has been demonstrated that the effect of ultrasound is to increase the migration rate and to sharpen the bands. Both of these are desirable for enhancing the separation power of gel electrophoresis. The principal reason for this phenomenon is that ultrasound decreases the intrinsic viscosity of DNA fragments and/or buffer solution, and decreases the DNA-agarose affinity, which cause DNA fragment bands to be retarded and to broaden. The study of these effects may be very useful to biochemists who are doing DNA sequencing. It should be pointed out that the ultrasonic frequency has not been optimized yet in this dissertation. It is reasonable to expect that by adjusting the ultrasound frequency, power, and location, one may be able to magnify these effects to introduce unique selectivity in DNA separations. 
ADDITIONAL REFERENCES

1. Izmailov, N. A.; Schraiber, M. S. Farmatsiya 1938, 3. 2 .

2. Meinhard, J. E.; Hall, N. F. Anal. Chem. 1949, 21, 185.

3. Kirchner, J. G.; Miller, J. M. ; Keller, G. J. Anal. Chem. 1951, 23, 420 .

4. Miller, J. M.; Kirchner, J. G. Anal. Chem. 1952, 24, 1480.

5. Miller, J. M.; Kirchner, J. G. Anal. Chem. 1954, 26, 2002 .

6. Stahl, E. Chemiker Ztg. 1958, 82, 323.

7. Stahl, E. Thin Layer Chromatography; Springer-Verlag: Berlin, 1965.

8. Kirchner, J. G. Thin Layer Chromatography; WileyInterscience: New York, 1967.

9. Touchstone, J. C.; Sherma, J. Techniques and Applications of Thin Layer Chromatography; John Wiley and Sons: New York, 1985.

10. Dallas, F. A. A.; Read, H.; Ruane, R. J.; Wilson, I. D. Recent Advances in Thin-Layer Chromatography; Plenum Press: New York, 1987.

11. Grinberg, N. Modern Thin-Layer Chromatography; Marcel Dekker: New York, 1990. 
12. Geiss, F. Fundamentals of Thin Layer Chromatography; Verlag: Heideiberg, 1987.

13. Poole, C. F.; Schuette, S. A. Contemporary practice of Chromatography; Elsevier: Amsterdam, 1984.

14. Jaenchen, D. E.; Issaq, H. J. I. Liquid Chromatogr. $1988,11,1941$.

15. Poole, C. F.; Poole, S. K. Anal. Chem. 1989, 61, $1257 \mathrm{~A}$.

16. Touchstone, J. C. "Modern Thin Layer Chromatography"; In Ahuja, S., Ed.; Ultratrace Analysis of Pharmaceuticals and other compounds of Interest; John Wiley and Sons: New York, 1986.

17. Yeung, E. S. Detectors for Liquid Chromatography; John Wiley and Sons: New York, 1986.

18a. Yeung, E. S.; Steenhoek, L. E.; Woodruff, S. D.; Kuo, J. C. Anal. Chem. 1981, 52, 1399.

18b. Kuo, J. C.; Yeung, E. S. J. Chromatogr. 1981, 223, 321.

18c. Kuo, J. C.; Yeung, E. S. J. Chromatogr. 1982, 253, 199.

19. Horvai, G.; Pungor, E. "Electrochemical Detectors in HPLC and Ion Chromatography"; In Zielinski, w. L. Jr., Ed.; Critical Reviews in Analytical Chemistry; CRC Press, Inc.: Boca Raton, 1989; Vol. 21, p. 1. 20a. Johnson, D. C. Nature (London) 1986, 321, 451. 
20b. Jundik, P.: Hadaad, P. R.; Sturrock, P. E. "Electrochemical Detectors for Ion Chromatographic Analysis: A Critical Review"; In Zielinski W. L. Jr., Ed.; Critical Reviews in Analytical Chemistry; CRC Pess, Inc.: Boca Raton, 1988; Vol. 20, p. 1.

21. Touchstone, J. C. J. Chromatogr. Sci. $1988,26,645$.

22. Jaenchen, D. E. American Lab. 1988, 3, 66 .

23. Touchstone, J. C.; Dobbins, M. F. Practice of Thin Layer Chromatography; John Wiley and Sons: New York, 1978.

24. Jork, H.; Funk, W.; Fischer, W.; Wimmer, H. ThinLayer Chromatography; VCH: Weinheim, 1990; Vol. 1a.

25. Fried, B.; Sherma, J. Thin-Layer Chromatography; Marcel Dekker: New York, 1986.

26. Bicking, M. K. L.; Kinseley, R. N. : Svec, H. J. Anal. Chem. 1983, 55, 200.

27. Huie, C. W.; Williams, W. R. Anal. Chem. 1989, 61, 2288 .

28. Guilbout, G. G. Practical Fluorescence Theory, Methods and Techniques; Marcel Dekker: New York, 1973.

29. Touchstone, J. C.: Sherma, J. Densitometry in Thin Layer Chromatography; John Wiley and Sons: New York, 1979.

30. Hurtubise, R. J. Solid Surface Luminescence Analysis. 
Theory, Instrumentation, Applications; Marcel Dekker: New York, 1981.

31. Cotgreave, T.; Lynes, A. J. Chromatogr. 1967, 30, 117 .

32. Linenberg, A.; Novick, O. Isr. J. Chem. 1970, 8, 68 .

33. Mangold, H. K.; Mukherjee, K. D. I. Chromatogr. Sci. $1975,13,398$.

34. Mukherjee, K. D. "Tublilar TLC"; In Paoletti, R.; Jacini, G.; Porcellati, R., Eds.; Lipids; Raven: New York, 1976; Vol. 2, p. 361.

35. Okumura, T.; Kadono, T.; Iso'o, A. J. Chromatogr. $1975, \underline{102}, 329$.

36. Mukherjee, K. D. "Quantitative Thin-Layer Chromatography wlth Flame Ionization Detection"; In Treiber, L. R., Ed; Quantitative Thin-Layer Chromatography and Its Industrial Applications; Marcel Dekker: New York, 1987.

37. Ranny, M. Thin-Layer Chromatography with Flame Ionization Detection; Reidel: Boston, 1987.

38. Sebedio, J. L.; Astorg, P. O.; Septier, C.; Grandgirard, A. I. Chromatogr. 1987, 405, 371.

39. Larson, J. R.; Pfeiffer, C. D. Anal. Chem. 1983, 5ㅗ, 393.

40. Takeuchi, T.; Yeung, E. S. I. Chromatogr. 1986, 366, 145 . 
41. Takeuchi, T.; Yeung, E. S. I. Chromatogr. 1986, 370, 83.

42. Takeuchi, T.; Ishii, D. I. Chromatogr. 1987, 403, 324.

43. Pfeffer, W. D.; Takeuchi, T.; Yeung, E. S. Chromatographia $1987, \underline{24}, 123$.

44. Jardy, A.; Caude, M.; Diop, A.; Curvale, C.; Rosset, R. I. Chromatogr. $1988, \underline{439}, 137$.

45. Schill, G.; Crommen, J. Trends in Anal. Chem. 1987, 6, 111 .

46. Takeuchi, T.; Suzuki, E.; Ishii, D. I. Chromatogr. $1988,447,221$.

47. Arvidsson, E.; Hackzell, L.; Schill, G.; Westerlund, D. Chromatographia $1988, \underline{25}, 430$.

48. Jurkiewicz, K. I. Liquid Chromatogr. 1989, 12, 2145.

49. Kuhr, W. G.; Yeung, E. S. Anal. Chem. 1988, 60, 1832 .

50. Kuhr, W. G.; Yeung, E. S. Anal. Chem. 1988, 60, 2642 .

51. Hogan, B. L.; Yeung, E. S. I. Chromatogr. Sci. 1990, 28, 15 .

52. Gross, L.; Yeung, E. S. I. Chromatogr. 1989, 480, 169.

53. Garner, T. W. ; Yeung, E. S. J. Chromatogr. 1990, in press.

54. Small, H.; Miller, T. E., Jr. Anal. Chem. 1982, 54, 462 . 
55. Bobbitt, D. R. ; Yeung, E. S. Anal. Chem. 1984, 56, 1577 .

56. Bobbitt, D. R.; Yeung, E. S. Anal. Chem. 1985, 57, 271.

57. Mho, S. I.; Yeung, E. S. Anal. Chem. 1985, 57, 2253.

58. Yeung, E. S. AcC. Chem. Res. 1989, 22, 125.

59. Ishii, D.; Takeuchi, T. I. Liquid Chromatogr. 1988, 11, 1865 .

60. Kawazumi, H.; Yeung, E. S. Appl. Spectrosc. 1988, 42, 1228 .

61. Kawazumi, H. ; Yeung, E. S. Appl. Spectrosc. 1989, 43, 249 .

62. Imaeda, K.; Ohsawa, K.; Uchiyama, K.; Nakamura, S.; Tokieda, T. Anal. Sci. 1986, 2,9 .

63. Chen, I. I.; Morris, M. D. Anal. Chem. 1984, 56, 19.

64. Chen, I. I.; Morris, M. D. Anal. Chem. 1984, 56, 1674 .

65. Kaiser, R. E. Proceedings of the Third Interational Symposium on Instrumental HPTLC; Institute for Chromatography: Bad Burkheim, West Germany, 3.985 ; p. 427 .

66. Chang, T. T.; Lay, J. O.; Francel, R. J. Anal. Chem. $1984, \underline{56}, 109$.

67. Ramaley, L.; Vaughn, M.-A.; Jamieson, W. D. Anal. Chem. $1985,57,353$. 
68. Kraft, R.; Otto, A.; Zoepfl, H. J.; Etzold, G. Biomed. Environ. Mass Spectrom. 1987, 14, 1.

69. Busch, K. Trends in Anal. Chem. 1987, 6, 95.

70. Belchamber, R. M.; Read, H.; Roberts, J. D. M. I. Chromatogr. 1987, 395, 47.

71. Lengyel, B. A. Introduction to Laser Physics; Wiley: New York, 1966.

72. Moenke, H.; Moenke-Blankenburg, L. Laser MicroSpectrochemical Analysis; Crane: New York, 1968.

73.. Omenetto; N. Analytical Laser Spectroscopy; John Wiley and Sons: New York, 1979.

74. Piepmeier, E. H. Analytical Applications of Lasers; John Wiley and Sons: New York, 1986.

75. Letokhov, V. S. Laser Analytical Spectrochemistry; Adam Hilger: Boston, 1986.

76. Moenke-Blankenburg, L. Laser Microanalysis; John Wiley and Sons: New York, 1989.

77. Berman, M. R. ; Zare, R. N. Anal. Chem. 1975, 47, 1200.

78. Bicking, M. K. L.; Kniseley, R. N.; Svec, H. J. Anal. Chem. 1983, 55, 200.

79. Belenkii, B. G.: Gankina, E. S. I. Chromatogr. 1986, 365,315 .

80. Sepaniak, M. J.; Yeung, E. S. Anal. Chem. 1977, 49, 1554 . 
81. Huff, P. B.; Tromberg, B. J.; Sepaniak, M. J. Anal. Chem. $1982, \underline{54}, 946$.

82. Huff, P. B.; Sepaniak, M. J. Anal. Chem. 1983, 55, 1992 .

83. Zhu, J. ; Yeung, E. S. I. Chromatogr. 1989, 463, 139.

84. Zhu, J.; Yeung, E. S. Anal. Chem. 1989, 61, 1906.

85. Tiselius, A. Trans. Frraday Soc. $1937, \underline{33}, 524$.

86. Deyl, Z.; Everaerts, F. M.; Prusik, Z.; Svendsen, P. J. Electrophoresis; Elsevier: Ansterdam, 1979.

87. Stellwagen, N. C. "Electrophoresis of DNA in Agarose and Polyacrylamide Gels"; In Chrambach, A.; Dunn, M. J.; Radola, B. J., Eds.; Advances in Electrophoresis; VCH: Weinheim, 1987; Vol. 1 .

88. Ogden, R. C.; Adams, D. A. "Electrophoresis in Agarose and Acrylamide Gels"; In Berger, S. L.; Kimmel, A. R., Eds.; Methods in Enzymology; Academic Press: New York, 1987; Vol. 152.

89. Andrews, A. T. Electrophoresis; Clarendon: Oxford, 1986.

90. Catsimpoolas, N. Methods of Protein Separation; Plenum Press: New York, 1976; Vol. 2, p. 145.

91. Stellwagen, N. Biopolymers 1985, 24, 2243.

92. Ogston, A. G. Trans. Faraday Soc. 1958, 54, 1754.

93. Rodbard, D. ; Chrambach, A. Proc. Nat. Acad. Sci. USA $1970,65,970$. 
94. Rodbard, D.; Chrambach, A. Anal. Biochem. 1971, 40, 95.

95. Chrambach, A.; Rodbard, D. Science 1971, 170, 440.

96. Ansinge, W.; Barker, R. J. Biochem. Biophys. Method $1984,9,33$.

97. Neidhardt, F. C.; Phillips, T. A. In Two-Dimensional Gel Electrophoresis of Proteins; In Celis, J. E.; Bravo, R., Eds.; Academic Press: Orlando, 1984; Chapter 13.

98. Endler, A. T.; Hanash, S. Two-Dimensional

Electrophoresis; VCH: Weinheim, 1989.

99. Schwartz, D. C.; Cantor, C. R. Cell 1984, 37, 67.

100. Chen, K.; Lance, B. K.; Yeung, E. S. Manuscript in preparation. Dept. Chem., Iowa State University.

101. Hogan, B. L.; Yeung, E. S. Appl. Spectrosc. 1.989, 43, 349 .

102. Hervet, H.; Bean, C. P. Biopolymers $1987, \underline{26}, 727$.

103. Cohen, A. S.; Najarian, D.; Smith, J. A.; Karger, B. L. I. Chromatogr. 1988, 458, 323 .

104. Karger, B. L.; Cohen, A. S.; Gurtman, A. I. Chromatogr. 1989, 492, 585 .

105. Drossman, H.; Luckey, J. A.; Kostichka, A. J.; D'cunha, J.; Smith, L. M. Anal. Chem. 1990, 62, 900. 106. Hinkley, J. O. N. J. Chromatogr. 1975, 109, 209. 107. Brown, J. F.; Hinkley, J. O. N. I. Chromatogr. 1975, 
109,218 .

108. Ewing, A. G.; Wallingford, R. R. ; Olefirowicz, T. M. Anal. Chem. 1989, 61, 292A.

109. Foret, F.; Bocek, P. "Capillary Electrophoresis"; In Chrambach, A.; Dunn, M. J.; Radola, B. J.; Eds.; Advances in Electrophoresis; VCH: Weinheim, 1989; Vol. 3 .

110. Gordon, M. J.; Huang, X.; Pentoney, S. L., Jr.; Zare, R. N. Science $1988, \underline{242}, 224$.

111. Chen, C. Y.; Morris, M. D. Appl. Spectrosc. 1.988, 42, 515 .

112. Smith, R. D. ; Olivares, J. A.; Nguyen, N. T.; Udseth, H. R. Anal. Chem. 1988, 60, 436 .

113. Smith, R. D.; Udseth, H. R. Nature 1988, 331, 638.

114. Wallingford, R. A. ; Ewing, A. G. I. Chromatocr. 1988, $\underline{441}, 299$.

115. Huang, X.; Pang, T. K. ; Gordon, M. J.; Zare, R. N. Anal. Chem. 1987, 59, 2747.

116. Wallingford, R. A. ; Ewing, A. G. "Capillary Electrophoresis"; In Giddings, J. C.; Grushka, E.; Brown, P. R., Eds. Adv. Chromatogr.; Marcel Dekker: New York, 1989; Vol. 29.

117. Burton, D. E.; Sepaniak, M. J.; Maskarinec, M. P. J. Chromatogr. Sci. $1986, \underline{24}, 347$.

118. Terabe, S.; Otsuka, K. ; Ichikawa, K.: Tsuchiya, A.; 
Ando,' T. Anal. Chem. 1984, 5ㅌ, 111.

119. Kuhr, W. G. Anal. Chem. 1990, 62, 403R.

120. Zhu, M. ; Hansen, D. L. ; Burd, S. ; Gannon, F. I. Chromatogr. $1989, \underline{480}, 311$.

121. Chin, A. M. Research News 1990, 2, 13.

122. Curie, J.; Curie, P. Compt. Rend, 1881, 93, 1137.

123. Cracknell, A. P. Ultrasonics; Wykeham: New York, $1980 ;$ p. 74 .

124. Rawson, F. F. Phys. Bull. 1987, 38, 255.

125. Suslick, K. S. Ultrosound; VCH: New York, 1988.

126. Brown, B. ; Gordun, D. Ultrasonic Techniques in

Biology and Medicine; Charles c. Thomas: Springfield, 1967.

127. Weissler, A. I. Chem. Educ. 1948, 25, $2 \varepsilon$.

128. Mason, T. J.; Lorimer, J. P. Sonochemistry; Ellis

Horwood: New York, 1988.

129. Bremner, D. Chemistry in Britain 1986, July, 633 .

130. Dunken, H.; Pforr, G.; Mikkeleit, W. Z. Chemistry $1964,4,237$.

131. Dunken, H.; Pforr, G.; Mikkeleit, W.; Geller, K. Spectrochim. Acta 1964, 20, 1531.

132. Kirsten, W. J.; Bertilsson, G. O. B. Anal. Chem. $1966, \underline{38}, 648$.

133. Van Rensberg, H. C.; Zeeman, P. B. Anal. Chem. Acta $1968, \underline{43}, 173$. 
134. Denton, M. B.; Swartz, D. B. Rev. Sci. Instrum. 1974, 45,81

135. Asher, R. C. Ultrasonics $1987, \underline{25}, 17$.

136. Synovec, R. E.; Yeung, E. S. I. Chromacogr. 1987, $\underline{388}, 105$. 


\section{ACKNOWLEDGEMENT}

This work was performed at Ames Laboratory under contract no. W-7405-eng-82 with the U.S. Department of Energy. The United States government has assigned the DOE Report number IS-T 1502 to this thesis.

First of all, I want to thank Dr. Edward S. Yeung, my major professor, for offering the opportunity and supporting me to do researches in this famous detector and spectroscopy group. His expert guidance, thoughtful supervision make this dissertation be possible. His dedication of science, high efficiency of working and the virtue of patience are good models for me.

I want to thank Dr. Donald J. Graves for serving as my minor professor. His enlighting teaching and special guidance in biochemistry really broaden my knowledge and stimulate my interests in bioanalysis.

I also want to express my thanks to the professors who served on my committee: Dr. Dennis C. Johnson, Dr. Robert S. Houk, and Dr. Cheuk-Yiu Ng. They devoted their precious time to help me finish all the tedious work during this program.

I would like to thank all the graduate students and postdocs in this group, who gave me a lot of help in my research work. They are so considerate in sharing 
equipment, space, and so enthusiastic in sharing their knowledge and experience.

I am also grateful to Lance B. Koutny for the laserbased indirect fluorometric detection project, to Hirofumi Kawazumi for helping me write computer programs. Finally, I would like to thank my wife, Honglan Shi, for her love, understanding and support, for taking care of our two lovely children, which made a special contribution to this course of work. 

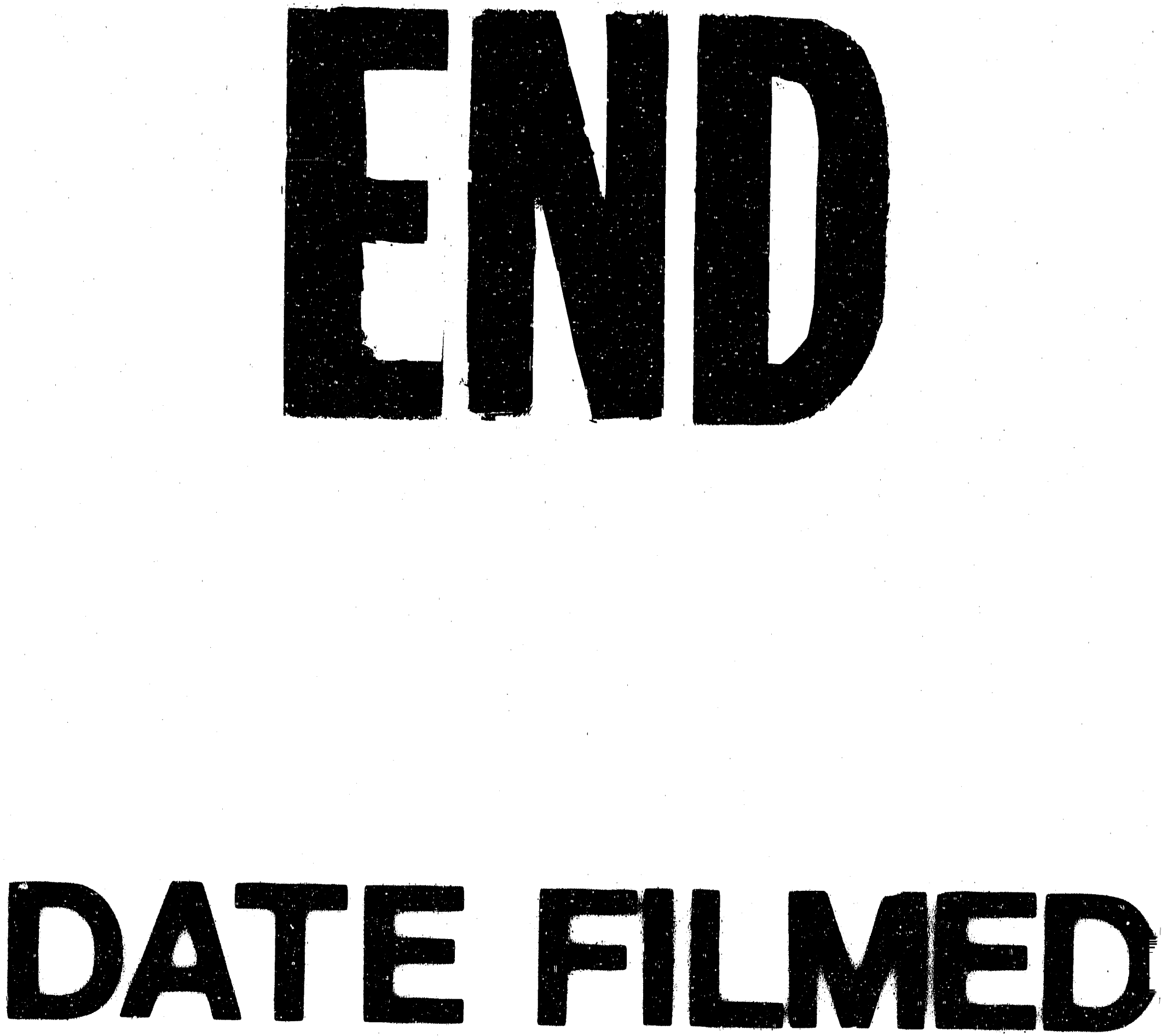

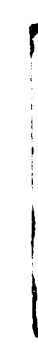
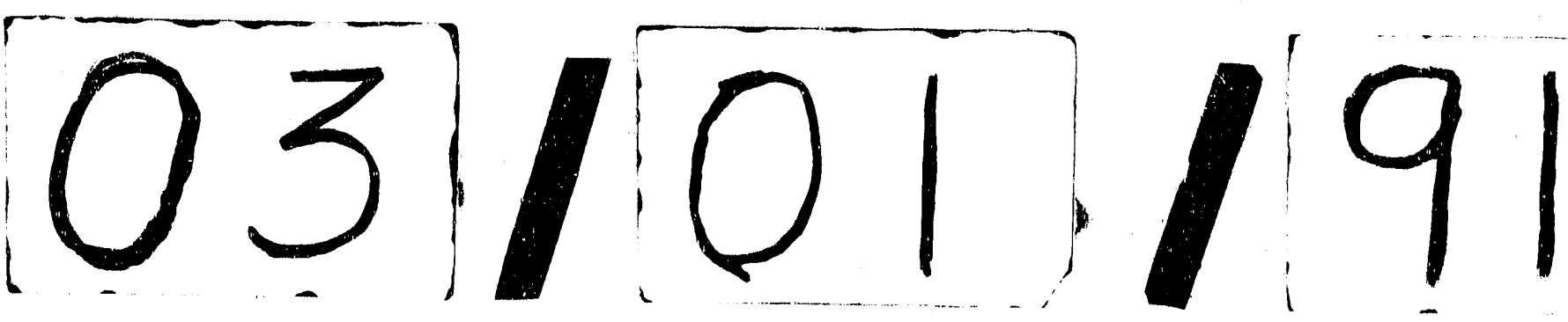


\section{.}

